Florida International University FIU Digital Commons

FIU Electronic Theses and Dissertations

University Graduate School

$11-7-2011$

\title{
Thermal Imaging As A Biometrics Approach To Facial Signature Authentication
}

Ana M. Guzman Tamayo

Florida International University, ana.guzman2@fiu.edu

DOI: $10.25148 /$ etd.FI11120916

Follow this and additional works at: https://digitalcommons.fiu.edu/etd

\section{Recommended Citation}

Guzman Tamayo, Ana M., "Thermal Imaging As A Biometrics Approach To Facial Signature Authentication" (2011). FIU Electronic Theses and Dissertations. 539.

https://digitalcommons.fiu.edu/etd/539

This work is brought to you for free and open access by the University Graduate School at FIU Digital Commons. It has been accepted for inclusion in FIU Electronic Theses and Dissertations by an authorized administrator of FIU Digital Commons. For more information, please contact dcc@fiu.edu. 


\section{FLORIDA INTERNATIONAL UNIVERSITY}

Miami, Florida

\section{THERMAL IMAGING AS A BIOMETRICS APPROACH TO FACIAL SIGNATURE}

\section{AUTHENTICATION}

A dissertation submitted in partial fulfillment of the

requirements for the degree of

DOCTOR OF PHILOSOPHY

in

ELECTRICAL ENGINEERING

by

Ana M. Guzmán Tamayo 
To: Dean Amir Mirmiran

College of Engineering and Computing

This dissertation, written by Ana M. Guzman Tamayo, and entitled Thermal Imaging As A Biometrics Approach To Facial Signature Authentication, having been approved in respect to style and intellectual content, is referred to you for judgment.

We have read this dissertation and recommend that it be approved.

$\begin{array}{r}\hline \text { Armando Barreto } \\ \hline \text { Jean Andrian } \\ \hline \text { Naphtali Rishe } \\ \hline \text { Malek Adjouadi, Major Professor }\end{array}$

Date of Defense: November 7, 2011

The dissertation of Ana M. Guzmán Tamayo is approved.

Dean Amir Mirmiran

College of Engineering and Computing

Dean Lakshmi N. Reddi

University Graduate School

Florida International University, 2011 


\section{DEDICATION}

To the most amazing women in my life, my mother Juana Tamayo Arenas, whose unconditional love, conscientiousness, intelligence, and sacrifices made it possible for me to have an education. To my grandmother, Guadalupe Arenas who raised me with loving care. To Diana Garmendia, who gave me the opportunity to see a world full of infinite possibilities and kindness, and to Imelda Pleitez who has been an unconditional friend for many years. 


\section{ACKNOWLEDGMENTS}

I would like to express my deepest gratitude to my advisor, Dr. Malek Adjouadi, for his patience, hard work, advice, and support during my graduate studies. I thank him for giving me the opportunity to be part of the Center for Advanced Technology and Education (CATE) where I have learnt about research, education, and human relations. I extend my appreciation to my committee members, Dr. Jean Andrian, Dr. Armando Barreto, and Dr. Naphtali Rishe. I thank you for the help that you have provided through the preparation of this dissertation and my career at Florida International University.

I would also like to thank Jin Wang, Javier Delgado, Anas Salah Eddin, and Mohammed Goryawala for their help during the preparation of this manuscript and through the last stages of my research. I also thank all the volunteers whose thermal infrared images made my research possible.

Finally I thank the National Science Foundation under grants CNS-1042341, CNC-0959985, and HRD-0833093. 
ABSTRACT OF THE DISSERTATION

THERMAL IMAGING AS A BIOMETRICS APPROACH TO FACIAL SIGNATURE

AUTHENTICATION

by

Ana M. Guzmán Tamayo

Florida International University, 2011

Miami, Florida

Professor Malek Adjouadi, Major Professor

This dissertation develops an image processing framework with unique feature extraction and similarity measurements for human face recognition in the thermal mid-wave infrared portion of the electromagnetic spectrum. The goals of this research is to design specialized algorithms that would extract facial vasculature information, create a thermal facial signature and identify the individual. The objective is to use such findings in support of a biometrics system for human identification with a high degree of accuracy and a high degree of reliability. This last assertion is due to the minimal to no risk for potential alteration of the intrinsic physiological characteristics seen through thermal infrared imaging. The proposed thermal facial signature recognition is fully integrated and consolidates the main and critical steps of feature extraction, registration, matching through similarity measures, and validation through testing our algorithm on a database, referred to as C-X1, provided by the Computer Vision Research Laboratory at 
the University of Notre Dame.

Feature extraction was accomplished by first registering the infrared images to a reference image using the functional MRI of the Brain's (FMRIB's) Linear Image Registration Tool (FLIRT) modified to suit thermal infrared images. This was followed by segmentation of the facial region using an advanced localized contouring algorithm applied on anisotropically diffused thermal images. Thermal feature extraction from facial images was attained by performing morphological operations such as opening and top-hat segmentation to yield thermal signatures for each subject. Four thermal images taken over a period of six months were used to generate thermal signatures and a thermal template for each subject, the thermal template contains only the most prevalent and consistent features. Finally a similarity measure technique was used to match signatures to templates and the Principal Component Analysis (PCA) was used to validate the results of the matching process.

Thirteen subjects were used for testing the developed technique on an in-house thermal imaging system. The matching using an Euclidean-based similarity measure showed $88 \%$ accuracy in the case of skeletonized signatures and templates, we obtained $90 \%$ accuracy for anisotropically diffused signatures and templates. We also employed the Manhattan-based similarity measure and obtained an accuracy of $90.39 \%$ for skeletonized and diffused templates and signatures. It was found that an average $18.9 \%$ improvement in the similarity measure was obtained when using diffused templates. The 
Euclidean- and Manhattan-based similarity measure was also applied to skeletonized signatures and templates of 25 subjects in the $\mathrm{C}-\mathrm{X} 1$ database.

The highly accurate results obtained in the matching process along with the generalized design process clearly demonstrate the ability of the thermal infrared system to be used on other thermal imaging based systems and related databases. A novel user-initialization registration of thermal facial images has been successfully implemented. Furthermore, the novel approach at developing a thermal signature template using four images taken at various times ensured that unforeseen changes in the vasculature did not affect the biometric matching process as it relied on consistent thermal features. 


\section{TABLE OF CONTENTS}

CHAPTER $\quad$ PAGE

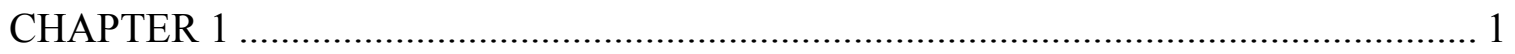

1.1 General Statement of the Problem Area …................................................. 1

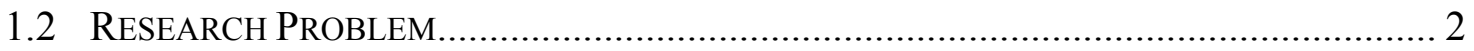

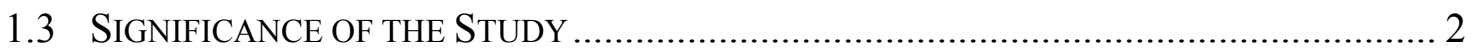

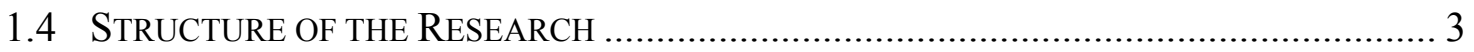

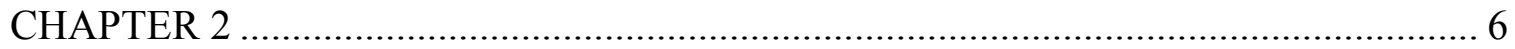

2.1 FUNDAMENTALS OF THERMAL INFRARED IMAGING …......................................... 6

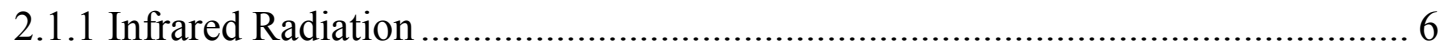

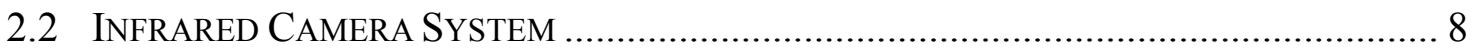

2.2.1 Camera Design and Image Formation .................................................... 10

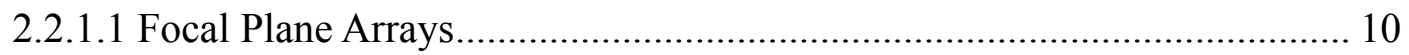

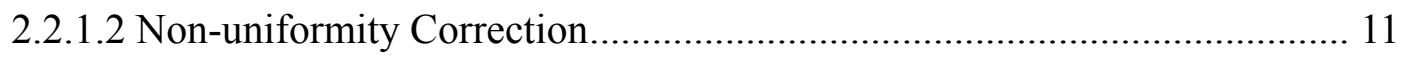

2.2.2 Detector Temperature Stabilization and Detector Cooling ................................ 11

2.2.3 Optics and Filters .................................................................................... 12

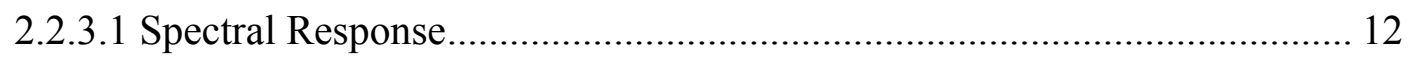

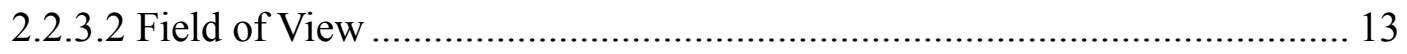

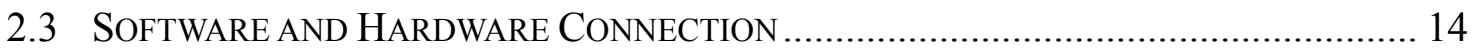

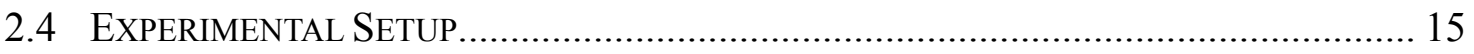

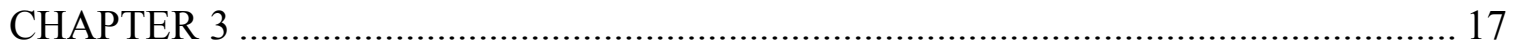

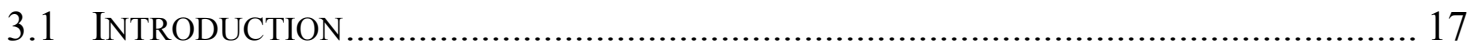

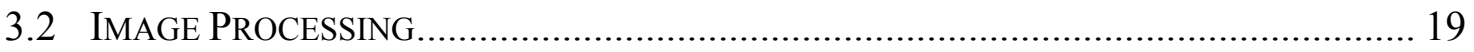

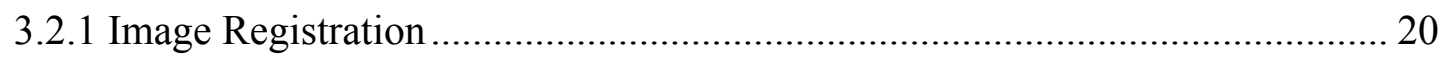

3.2.2 Thermal Signature Extraction ...................................................................... 24

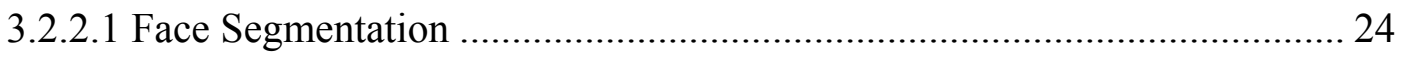

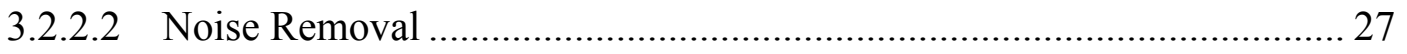

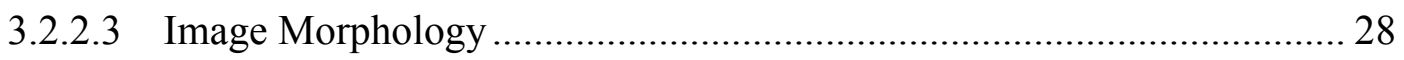

3.2.2.4 Signature Segmentation Post-Processing ............................................... 29

3.2.3 Generation of Thermal Signature Template ...................................................... 30

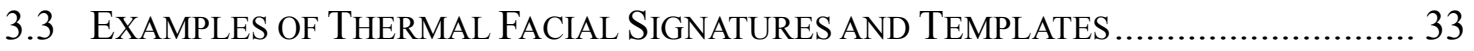

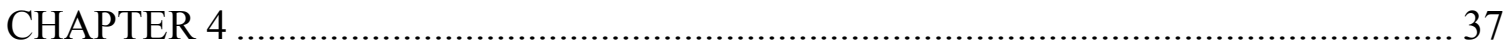

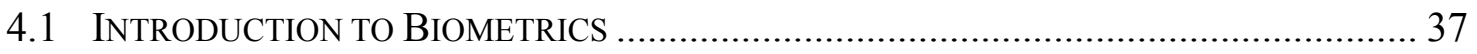

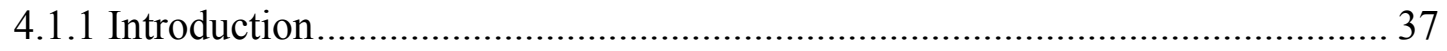

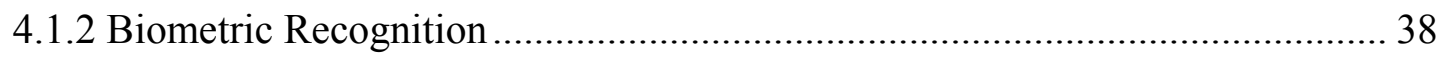




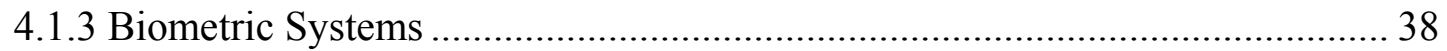

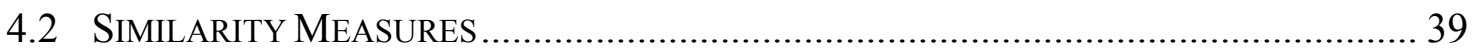

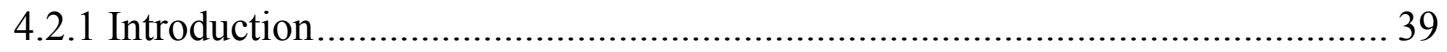

4.2.2 General definition of Similarity Measures..................................................... 40

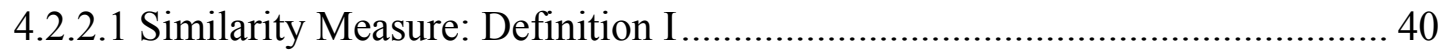

4.2.2.2 Similarity Measure: Definition II ............................................................. 41

4.2.2.3 Similarity Measure: Definition III............................................................. 44

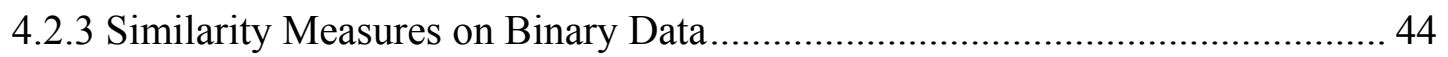

4.2.4 Similarity Measures on Numerical Data.................................................... 45

4.2.5 Similarity Measures Based in Dissimilarity ………………………………..... 46

4.3 Distance-Based Similarity Measure for Matching Thermal Signatures AND

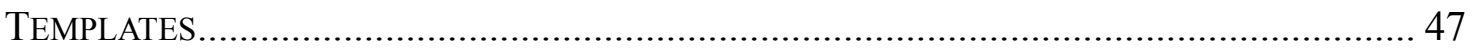

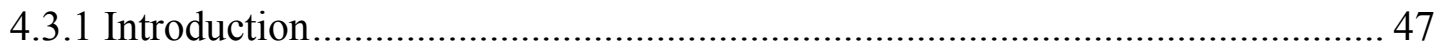

4.3.2 Distance-Based Similarity Measure............................................................... 47

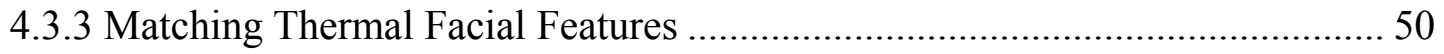

4.4 Partial Results of APPlied Similarity Measure............................................. 51

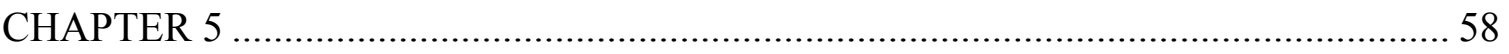

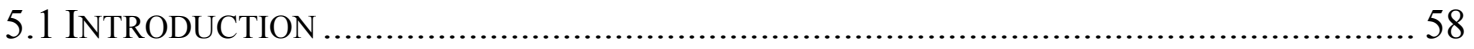

5.2 INTER-SUBJECT RESULTS FOR SKELETONIZED TEMPLATES AND SigNATURES ............ 58

5.2.1 Similarity Results for Matching Template to Templates................................. 58

5.2.2 Similarity Results for Matching the First Signature Set to Templates............ 60

5.2.3 Similarity Results for Matching the Second Signature Set to Templates ...... 63

5.2.4 Similarity Results for Matching the Third Signature Set to Templates .......... 66

5.2.5 Similarity Results for Matching the Fourth Signature Set to Templates ....... 68

5.3 INTER-SUBJECT RESULTS FOR DifFUSED TEMPLATE AND SIGNATURE …………...... 71

5.3.2 Similarity Results for Matching Diffused First Signature Set to Templates.. 72

5.3.3 Similarity Results for Matching Diffused Second Signature Set to Templates .. 75

5.3.4 Similarity Results for Matching Diffused Third Signature Set to Templates 77

5.3.5 Similarity Results for Matching Diffused Fourth Signature Set to Templates ... 80

5.3.5 Similarity Results for Matching Signatures to Modified Templates.............. 82

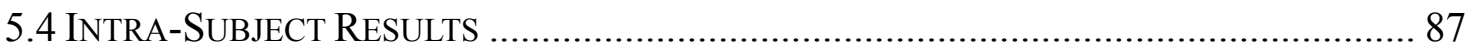

5.5 SiMILARITY RESULTS FOR THE C-X1 DATABASE.................................................... 90

5.6 VAlidation of Similarity Values Using Principle Component ANALYSIS ...... 94

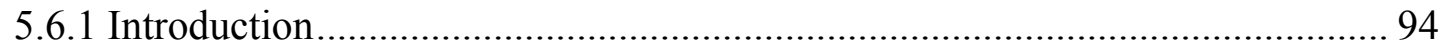

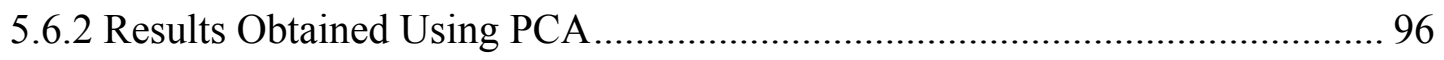

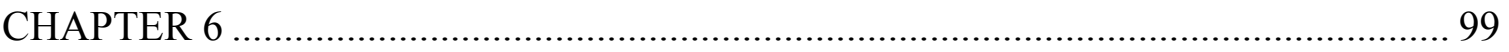

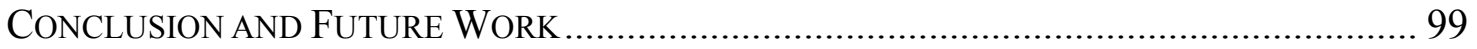




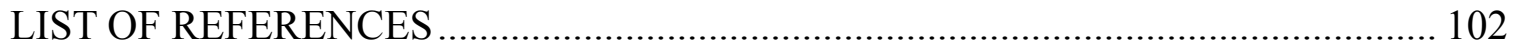

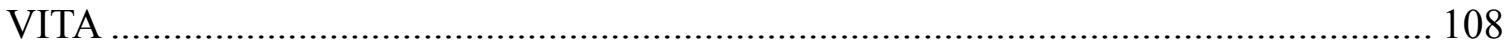




\section{LIST OF FIGURES}

FIGURE

PAGE

FIG. 2.1 THE ELECTROMAGNETIC SPECTRUM AND THE THERMAL INFRARED REGION [QI AND

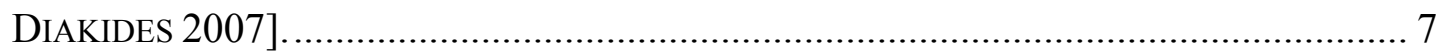

FIG. 2.2 BLOCK DIAGRAM OF AN IR CAMERA SYSTEM [COURTESY OF FLIR SYSTEMS IR

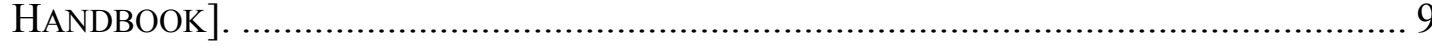

FIG. 2.3 MERLIN ${ }^{\text {TM }}$ MWIR CAMERA USED FOR DATA COLLECTION. [COURTESY OF FLIRTM

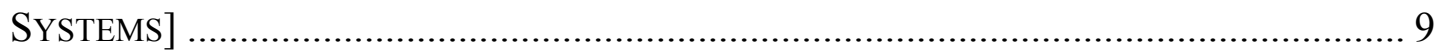

FIG. 2.4 BASIC COMPONENTS FOR (A) SCANNING SYSTEM AND (B) STARING SYSTEM

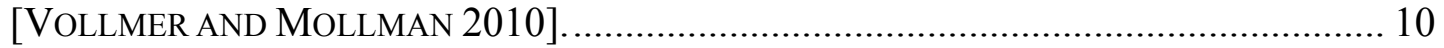

FIG. 2.5 SPECTRAL RESPONSE FOR VARIOUS IR CAMERAS.[COURTESY OF FLIR SYSTEMS] 13

Fig 2.6 CONNECTION CONFIGURATION BETWEEN THE MERLIN® MWIR CAMERA AND PC.

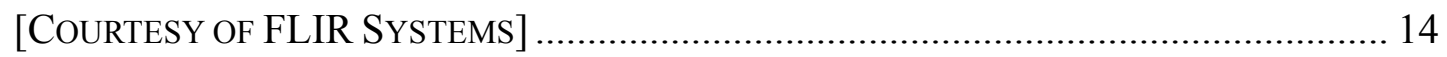

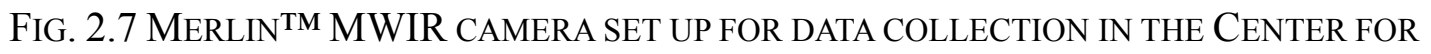

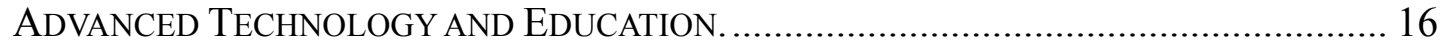

Fig. 3.1 HuMAN FACIAL VASCULAR NETWORK [MOXHAM ET AL., 2002] ........................... 18

FIG. 3.3 THE THREE MAIN STEPS TO GENERATE THERMAL FACIAL SIGNATURES AND

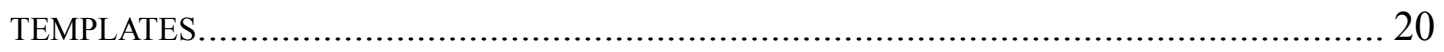

FIG. 3.4 REFERENCE THERMAL IMAGE............................................................... 23

FIG. 3.5 NON-REFERENCE THERMAL IMAGE. ....................................................... 23

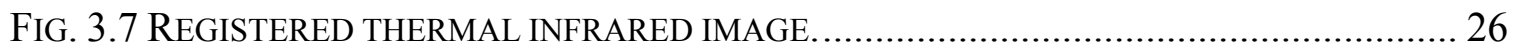

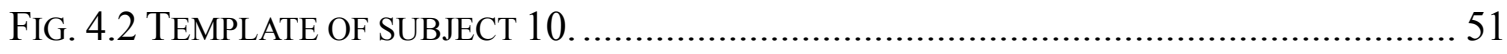

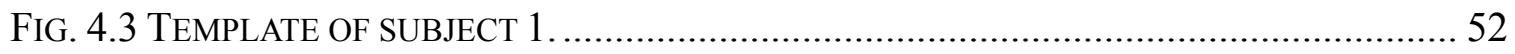

FIG. 4.3A TEMPLATE OF SUBJECT 1 (RED) OVERLAY WITH TEMPLATE OF SUBJECT 10 (WHITE).52 
Fig. 4.4 TEMPLATE OF SUBJECT 2

FIG. 4.4A TEMPLATE OF SUBJECT 2 (RED) OVERLAY WITH TEMPLATE OF SUBJECT 10 (WHITE).52

FIG. 4.5 TEMPLATE OF SUBJECT 3. 52

FIG. 4.5A TEMPLATE OF SUBJECT 3 (RED) OVERLAY WITH TEMPLATE OF SUBJECT 10 (WHITE).52

FIG. 4.6 TEMPLATE OF SUBJECT 4. 53

FIG. 4.6A TEMPLATE OF SUBJECT 4 (RED) OVERLAY WITH TEMPLATE OF SUBJECT 10 (WHITE).53

Fig. 4.7 TEMPLATE OF SUBJECT 5. 53

FIG. 4.7A TEMPLATE OF SUBJECT 5 (RED) OVERLAY WITH TEMPLATE OF SUBJECT 10 (WHITE).53

FIG. 4.8 TEMPLATE OF SUBJECT 6. 53

Fig. 4.8A TEMPLATE OF SUBJECT 6 (RED) OVERLAY WITH TEMPLATE OF SUBJECT 10 (WHITE).53

FIG. 4.9 TEMPLATE OF SUBJECT 7. 54

FIG. 4.9A TEMPLATE OF SUBJECT 7 (RED) OVERLAY WITH TEMPLATE OF SUBJECT 10 (WHITE).54

FIG. 4.10A TEMPLATE OF SUBJECT 8 (RED) OVERLAY WITH TEMPLATE OF SUBJECT 10

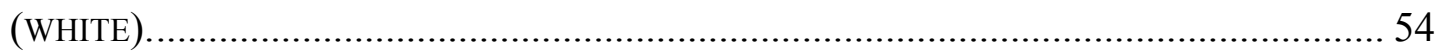

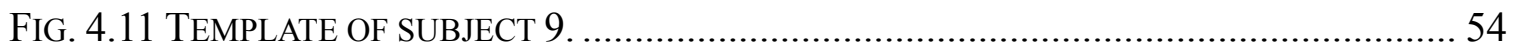

Fig. 4.11A TEMPLATE OF SUBJECT 9 (RED) OVERLAY WITH TEMPLATE OF SUBJECT 10 (WHITE) 54

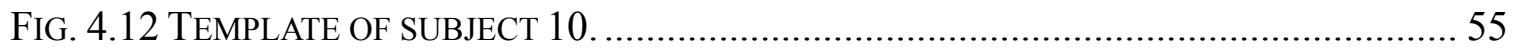

Fig. 4.12A TEMPLATE OF SUBJECT 10 (RED) OVERLAY WITH TEMPLATE OF SUBJECT 10..... 55

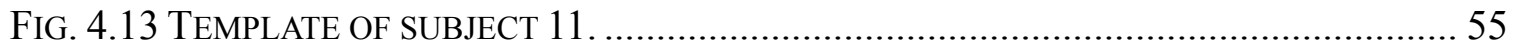

FIG. 4.13A TEMPLATE OF SUBJECT 11 (RED) OVERLAY WITH TEMPLATE OF SUBJECT 10

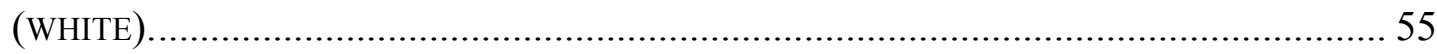




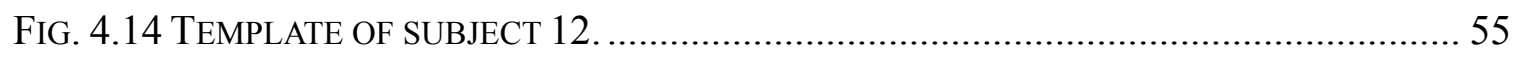

Fig. 4.14A TEMPLATE OF SUBJECT 12 (RED) OVERLAY WITH TEMPLATE OF SUBJECT 10

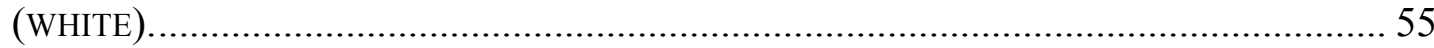

FIG. 4.15 THERMAL TEMPLATE OF SUBJECT 13 ........................................................... 56

Fig. 4.15A TEMPLATE OF SUBJECT 13 (RED) OVERLAY WITH TEMPLATE OF SUBJECT 10

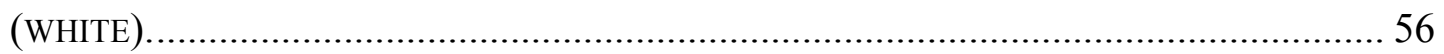

Fig. 5.1 OVERLAY OF TEMPLATES OF (A) SUBJECT 1 TO ITS OWN TEMPLATE AND (B) SUBJECT 1 TEMPLATE (WHITE) TO THE TEMPLATE OF SUBJECT 11 (RED)..................................... 60

Fig. 5.2 OVERLAY OF TEMPLATE (WHITE) AND SIGNATURE (RED) FOR POSITIVE MATCH OF (A) SUBJECT 12, (B) SUBJECT 6, AND (C) SUBJECT 1, WITH SIMILARITY VALUES OF [0.6158, $0.5043,0.4957]$ RESPECTIVELY. 62

Fig. 5.3 (A) SUBJECT 13's OVERLAY OF ITS OWN TEMPLATE (WHITE) AND SIGNATURE (RED), SIMILARITY VALUE 0.3097. (B) NEGATIVE MATCH OF THE SIGNATURE OF SUBJECT 13 TO THE TEMPLATE OF SUBJECT 12 WITH A SIMILARITY VALUE OF 0.3108 . 63

Fig. 5.4 OVERLAY OF TEMPLATE (WHITE) AND SIGNATURE (RED) FOR POSITIVE MATCH OF (A) SUBJECT 12, (B) SUBJECT 6, AND (C) SUBJECT 8 , WITH SIMILARITY VALUES OF [0.5661 $0.5806,0.4351]$ RESPECTIVELY. 65

Fig. 5.5 (A) SUBJECT 3'S OVERLAY OF ITS OWN TEMPLATE AND SIGNATURE, SIMILARITY VALUE 0.3001 . (B) NEGATIVE MATCH OF THE SIGNATURE OF SUBJECT 3 TO THE TEMPLATE OF SUBJECT 12 WITH A SIMILARITY VALUE OF 0.3541. 65

Fig. 5.6 OVERLAY OF TEMPLATE (WHITE) AND SIGNATURE (RED) FOR POSITIVE MATCH OF (A) SUBJECT 12, (B) SUBJECT 4, AND (C) SUBJECT 6, WITH SIMILARITY VALUES OF [0.5132, $0.4606,0.4556]$ RESPECTIVELY. 67

FIG. 5.7 (A) SUBJECT 9'S OVERLAY OF ITS OWN TEMPLATE AND SIGNATURE, SIMILARITY VALUE 0.2936 , (B) NEGATIVE MATCH OF THE SIGNATURE OF SUBJECT 9 TO THE TEMPLATE OF SUBJECT 7 WITH A SIMILARITY VALUE OF 0.3346. 68

Fig. 5.8 OVERLAY OF TEMPLATE (WHITE) AND SIGNATURE (RED) FOR POSITIVE MATCH (A) SUBJECT 12, (B) SUBJECT 4, AND (C) SUBJECT 6, SIMILARITY VALUES OF [0.5252, 0.4986, 
FIG. 5.9 (A) SUBJECT 4'S OVERLAY OF ITS OWN TEMPLATE AND SIGNATURE, SIMILARITY VALUE 0.3179 , (B) NEGATIVE MATCH OF THE SIGNATURE OF SUBJECT 9 TO THE TEMPLATE OF SUBJECT 12 WITH A SIMILARITY VALUE OF 0.3348. 70

FIG. 5.10 OVERLAY OF DIFFUSED TEMPLATE OF (A) SUBJECT 1 TO ITS OWN TEMPLATE AND (B) SUBJECT 1 TEMPLATE (WHITE) TO THE TEMPLATE OF SUBJECT 2 (RED).

FIG. 5.11 OVERLAY OF DIFFUSED TEMPLATE (WHITE) AND SIGNATURE (RED) FOR POSITIVE MATCH (A) SUBJECT 12, (B) SUBJECT 4, AND (C) SUBJECT 6, SIMILARITY VALUES [0.7569, $0.6466,0.6407]$ RESPECTIVELY.

FIG. 5.13 OVERLAY OF DIFFUSED TEMPLATE (WHITE) AND SIGNATURE (RED) FOR POSITIVE MATCH (A) SUBJECT 12, (B) SUBJECT 6, AND (C) SUBJECT 1, WITH SIMILARITY VALUES [0.7356, 0.7129, 0.5479] RESPECTIVELY.

FIG. 5.14 (A) SUBJECT 3 OVERLAY OF ITS OWN DIFFUSED TEMPLATE AND SIGNATURE, SIMILARITY VALUE 0.3163, (B) NEGATIVE MATCH SUBJECT 3 TO THE TEMPLATE OF SUBJECT 12, SIMILARITY VALUE OF 0.4249.

FIG. 5.15 OVERLAY OF DIFFUSED TEMPLATE (WHITE) AND SIGNATURE (RED) FOR POSITIVE MATCH (A) SUBJECT 12, (B) SUBJECT 5, AND (C) SUBJECT 6, WITH SIMILARITY VALUES $[0.6799,0.5787,0.5733]$ RESPECTIVELY.

FIG. 5.16 (A) SUBJECT 9 OVERLAY OF ITS OWN DIFFUSED TEMPLATE AND SIGNATURE, SIMILARITY VALUE 0.3026, (B) NEGATIVE MATCH SUBJECT 9 TO THE TEMPLATE OF SUBJECT 7, SIMILARITY VALUE OF 0.4072 .

FIG. 5.17 OVERLAY OF DIFFUSED TEMPLATE (WHITE) AND SIGNATURE (RED) FOR POSITIVE MATCH (A) SUBJECT , (6B) SUBJECT 12, AND (C) SUBJECT 3, WITH SIMILARITY VALUES $[0.6362,0.6240,0.5691]$ RESPECTIVELY.

FIG. 5.18 (A) SUBJECT 4 OVERLAY OF ITS OWN DIFFUSED TEMPLATE AND SIGNATURE, SIMILARITY VALUE 0.3895, (B) NEGATIVE MATCH OF SUBJECT 4 SIGNATURE TO THE TEMPLATE OF SUBJECT 6, SIMILARITY VALUE OF 0.4389.

FIG. 5.19 OVERLAY OF SIGNATURES AND MODIFIED TEMPLATES OF SUBJECT 1 83

FIG. 5.20 OVERLAY OF TWO-PIXELS THICK TEMPLATES AND SKELETONIZED SIGNATURES FOR 
SUBJECT 1.

FIG. 5.21 OVERLAY OF TWO-PIXELS THICK TEMPLATES AND SKELETONIZED SIGNATURES FOR

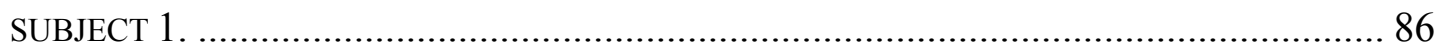

Fig. 5.22 SKELETONIZED SIGNATURES OF TWO DIFFERENT SUBJECTS IN C-X1................ 92

FIG. 5.23 THERMAL SIGNATURES OF A SUBJECT IN DATASET C-X1 AND WHOSE SIMILARITY VALUES RESULT IN A POSITIVE MATCH TO ITS TEMPLATE. ......................................... 93

FIG. 5.24 OVERLAY OF THERMAL SIGNATURE (RED) OF A SUBJECT IN DATASET C-X1 AND WHOSE SIMILARITY VALUES RESULT IN A POSITIVE MATCH TO ITS TEMPLATE (WHITE). 93

FIG. 5.25 OVERLAY OF TEMPLATES (WHITE) AND SIGNATURES (RED) WHOSE SIMILARITY VALUES PRODUCED A NEGATIVE MATCH. THE SIGNATURE IN 5.25A AND 5.25B IS THAT OF

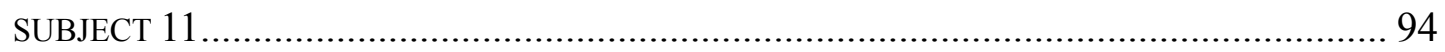




\section{LIST OF TABLES}

TABLE

PAGE

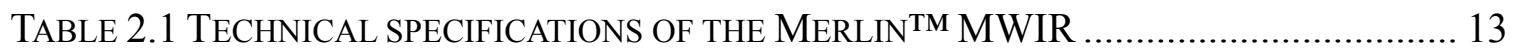

TABLE 4.1 TYPE 1 SIMILARITY MEASURES [LESOT AND RIFQI 2009]. .............................. 42

TABLE 4.2 TYPE 2 SIMILARITY MEASURES [LESOT AND RIFQI 2009]. .............................. 43

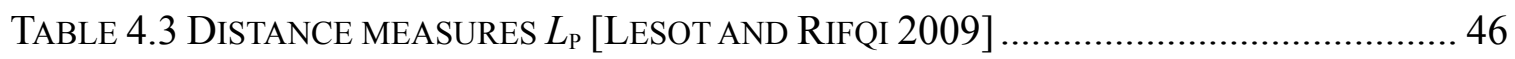

TABLE 4.4 NumERICAL EXAMPLES FOR (4.1), (4.3), AND (4.4) ...................................... 50

TABLE 4.5 NumERICAL EXAMPLES FOR (4.1), (4.3), AND (4.4)....................................... 50

TABLE 4.4 SimiLARITY VALUES FOR TEMPLATE VS. TEMPLATE ....................................... 56

TABLE 4.5 Similarity VALUES FOR SignATURE VS. TEMPLATE ...................................... 57

TABle 5.1 Similarity VALUES FOR STT $(\mathrm{A} \rightarrow \mathrm{B})$, WHERE $A$ AND $B$ ARE TEMPLATES.

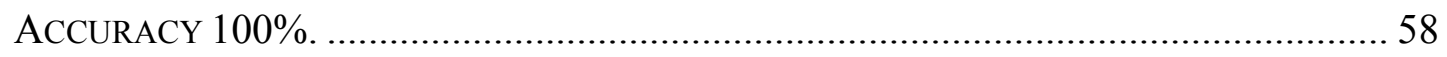

TABLE 5.2 SimiLARITY VALUES FOR SS1T(A $\rightarrow \mathrm{B})$, WHERE $A$ IS THE FIRST SIGNATURE SET,

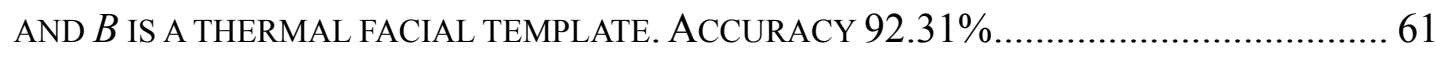

TABle 5.3 Similarity VALUES FOR SS2T $(\mathrm{A} \rightarrow \mathrm{B}), A$ IS THE SECOND SignATURE SET, AND

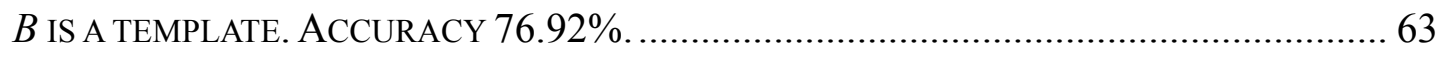

TABLE 5.4 SimiLARITY VALUES FOR SS3T $(\mathrm{A} \rightarrow \mathrm{B})$, WHERE $A$ IS THE THIRD SIGNATURE SET,

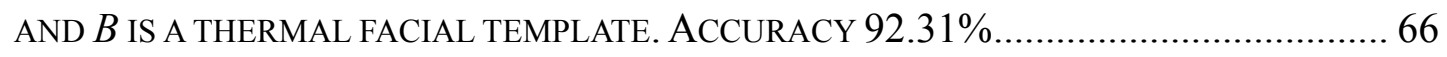

TABLE 5.5 Similarity VALUES FOR SS4T $(\mathrm{A} \rightarrow \mathrm{B})$, WHERE $A$ IS THE FOURTH SIGNATURE SET, AND $B$ IS A TEMPLATE. ACCURACY 92.31\%................................................ 69

TABLE 5.6 Similarity VALUES FOR STT $(\mathrm{A} \rightarrow \mathrm{B})$, WHERE $A$ AND $B$ ARE DIFFUSED

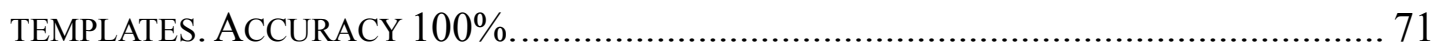

TABle 5.7 Similarity VALUES SS1T $(\mathrm{A} \rightarrow \mathrm{B})$, WHERE $A$ IS A DifFuSED Signature, AND $B$

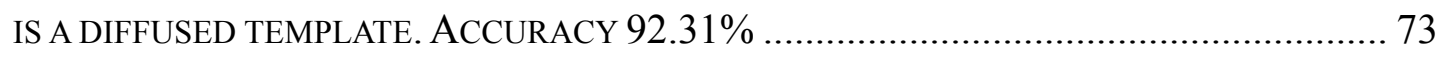


TABLE 5.8 SimiLARITY VALUES FOR SS2T $(\mathrm{A} \rightarrow \mathrm{B})$, WHERE $A$ IS THE DIFFUSED SIGNATURE, AND $B$ IS A DIFFUSED TEMPLATE. ACCURACY $84.61 \%$.

TABLE 5.9 SimILARITY VALUES SS3T $(\mathrm{A} \rightarrow \mathrm{B})$, WHERE $A$ IS A DIFFUSED SIGNATURE, AND $B$ IS A DIFFUSED TEMPLATE. ACCURACY $92.31 \%$..................................................... 78

TABle 5.10 Similarity VAlues SS4T $(\mathrm{A} \rightarrow \mathrm{B}), \quad A$ IS DifFUSED SignATURE, AND $B$ IS A

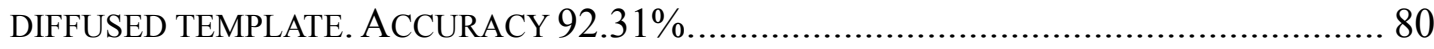

TABLE 5.11 ACCURACY FOR MATCHING SIGNATURES TO TEMPLATES CREATED USING 3 SIGNATURES (TEMPLATE-3S) AND 4 SIGNATURES (TEMPLATE-4S). TEMPLATES AND SIGNATURES ARE SKELETONIZED. 83

TABLE 5.12 ACCURACY VALUES FOR MATCHING SKELETONIZED SIGNATURES TO TEMPLATES

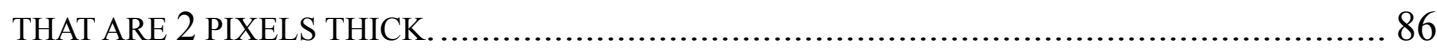

TABLE 5.13 INTRA-SUBJECT SIMILARITY VALUES. SKELETONIZED TEMPLATES AND SIGNATURES. 87

TABLE 5.14 INTRA-SUBJECT SIMILARITY VALUES. DIFFUSED TEMPLATES AND SIGNATURES.88

TABLE 5.15 PERCENTAGE INCREASE ON SIMILARITY VALUES. 88

TABLE 5.16 ACCURACY OF MATCHING FOR 4 DISTINCT SIGNATURES TAKEN AT DIFFERENT TIME TO THE SKELETONIZED AND DIFFUSED TEMPLATES IN THE DATABASE USING EUCLIDEAN DISTANCES. 89

TABLE 5.17 ACCURACY OF MATCHING FOR 4 DISTINCT SIGNATURES TAKEN AT DIFFERENT TIME TO THE SKELETONIZED AND DIFFUSED TEMPLATES IN THE DATABASE USING MANHATTAN DISTANCES.

TABLE 5.18 ACCURACY MATCHING FOR 4 DISTINCT SKELETONIZED SIGNATURE SETS AND TEMPLATES FOR 25 SUBJECTS IN THE C-X1 DATABASE. .......................................... 91

TABLE 5.18 PCA RESULTS FOR GROUP 1 AS THE TESTING DATA SET, Q=2 ....................... 96

TABLE 5.19 PCA RESULTS FOR GROUP 2 AS THE TESTING DATA SET, Q= $6 \ldots \ldots \ldots \ldots \ldots \ldots \ldots \ldots . . . . . . . . . . . .66$

TABLE 5.20 PCA RESULTS FOR GROUP 3 AS THE TESTING DATA SET, Q=6....................... 97 
TABLE 5.21 PCA RESUlTS FOR GROUP 4 AS THE TESTING DATA SET, Q=8 ........................ 98 


\section{CHAPTER 1}

Introduction

\subsection{General Statement of the Problem Area}

This research aims at establishing a system for human face recognition in the thermal Mid-Wave Infrared (MWIR) portion of the Electromagnetic (EM) spectrum. Facial recognition has been implemented in the visible light portion of the EM spectrum. Applications for face recognition can be found in the areas of entertainment, smart cards, information security, law enforcement and security [Zhao, 2003]. Multiple algorithms, techniques and systems have been created for face detection in areas that use cameras in the visible spectrum. Cameras in the MWIR portion of EM spectrum are available at a much higher cost than their visible band counterparts, thus much of the research done in human face recognition in the MWIR spectrum is at its infancy. Machine recognition of human faces has proven to be problematic due to light variability [Adini et al., 1997] and other factors like difficulty detecting facial disguises. The use of thermal MWIR for face recognition purposes solves the light variability problem as MWIR images are independent of light sources; MWIR imaging relies on the heat radiated by an object. Any foreign object on a human face such as a fake nose is detected, as foreign objects have a different heat emissivity than that of human skin. Human face recognition in the MWIR is a novel and challenging approach in the field of facial recognition. 


\subsection{Research Problem}

The objective of this study is to design an effective algorithm that will extract vasculature information, create a thermal facial signature and recognize the individual from a database. Techniques used in the visible spectrum will be explored and adapted to meet the requirements of MWIR images. In recent years, researchers have realized the potential of thermal MWIR imagery for human identification using the vein structure of hands [Lin and Fan 2004; Im et al. 2003], finger vein patterns [Shimooka and Shimizu 2004, Miura et al. 2004], and vein structure of the human face [Buddharaju et al., 2007]. The research performed by [Pavlidis et al., 2006] presents for the first time an algorithmic approach to face detection using MWIR images. In this study we intend to create a new system that will introduce new algorithms for feature extraction and recognition of human faces using MWIR imagery that will meet the critical standards of size and orientation independence. These algorithms should yield a robust and efficient feature extraction method, and higher recognition accuracy.

\subsection{Significance of the Study}

Face recognition tends to be the most appealing biometric procedure as it is the most natural process for human identification, it is the least obtrusive method and yet it remains the most challenging modality [Zhao et al. 2003, Bhattaharyya et al. 2009]. The first step in a biometric recognition or authentication system is face detection and feature extraction, which are necessary to locate the face position and obtain the face features in the image 
for further processing. The features obtained are then fed into the critical step of face recognition or authentication. The recognition or authentication process remains a challenging endeavor for researchers due to the myriad of faces that can be considered and the variability in the circumstances and ways under which the images of these faces are taken.

Therefore, feature extraction and subject recognition are considered the two focal points of this research. The system to be built will elaborate on how the learning and recognition phases are integrated into one system as it seeks higher recognition accuracy and faster processing time. The importance of this work relies on using the thermal MWIR portion of the EM spectrum for feature extraction and matching of human faces under circumstances that have proven unfavorable for visible spectrum systems.

\subsection{Structure of the Research}

The work presented in this dissertation consists of three major modules: (1) collection of MWIR images, (2) feature extraction, and (3) feature matching.

Chapter 2 explains the fundamental concepts of thermal infrared imaging, which include infrared radiation, electromagnetic waves, the electromagnetic spectrum, blackbody radiation and emissivity. After a brief review on the basic concepts of thermal infrared imaging this chapter introduces the main components of an infrared camera system. In this second section of the chapter the following concepts are explained: camera design and image formation, detector temperature stabilization and detector cooling, calibration, and 
camera software. Finally, the thermal infrared camera and computer installation are explained as well as the protocol followed to record thermal images from volunteer subjects.

Chapter 3 introduces the algorithm developed in this study for feature extraction using thermal images. The thermal MWIR camera provides the ability to directly image superficial blood vessels of the human face [Manohar 2006]. The pattern of the underlying blood vessels is unique to each individual, and the extraction of this vascular network can provide the basis for a feature vector. This module consists of facial segmentation (separation of background and face) and separation of facial features. The facial temperature information from a MWIR image is heterogeneous in nature; an appropriate segmentation method has been chosen to successfully separate background temperatures from facial temperatures. A robust localizing region-based segmentation method [Lankton and Tannenbaum 2008] is used for this purpose. In this algorithm, typical region-based active contour energies are localized in order to handle images with non-homogeneous foregrounds and backgrounds. The next step is the segmentation of thermal features, we use anisotropic diffusion to reduce noise and enhance edges in the image [Perona and Malik, 1990]. This is followed by a white-top hat segmentation technique to enhance the bright objects, which correspond to facial vasculature. Using morphological operators a thermal facial signature is created. We also generate a template, composed of four signatures, for each subject. The signature and the template are then 
used in the feature matching module.

Chapter 4 begins by explaining the concept of biometrics, its importance in our society and presents an overview of current technology in the field. The field of biometrics is closely related to the main work presented in this dissertation as it is our goal to use our thermal facial signatures to create a robust multimodal biometric system [Chen et al. 2010]. The second section of chapter 4 introduces the concept of similarity measures (SMs) for data classification. Basically, there are two types of SMs, type I and type II; section 4.2 will explain the difference between these SMs. In this section we'll also present the SM used for the recognition of the signatures and templates described in chapter 2 .

In chapter 5 we present and discuss the results obtained after applying the SM algorithm presented in chapter 4 to the signatures and templates in the database created for this study. We also present similarity results for 25 subjects chosen randomly from the data set C-X1, provided by the Computer Vision Research Laboratory at the University of Notre Dame. Chapter 6 concludes the dissertation, describes potential improvements and future research directions so that the subject recognition system could be extended to more application fields. 


\section{CHAPTER 2}

Thermal Infrared Imaging and Experimental Set-Up

\subsection{Fundamentals of Thermal Infrared Imaging}

Thermal infrared (IR) imaging, also referred to as thermography, is a field that used to be only associated with military and astronomy applications. However, in the past two decades the field has been expanded to applications in medicine, biometrics, computer vision, building maintenance, and many others. This chapter will briefly describe the main concepts on thermography, the components of an IR camera, the equipment configuration for this study, and the protocol used to collect data.

\subsubsection{Infrared Radiation}

Visible light, ultraviolet radiation, X-Ray radiation, IR radiation and so on are described as EM waves in the field of physics. A wave is the disturbance of a continuous medium that propagates with a fixed shape at constant velocity. The spatial periodicity is called wavelength, $\lambda$ (given in meters, micrometers, nanometers, etc.), the transient periodicity is called period of oscillation, $T$ (given in seconds), the reciprocal of $T(1 / T)$ is the frequency, $v$ (given in $\mathrm{Hz}$ ). The multiplication of frequency and wavelength give the speed, $c$, of the wave. The speed of propagation depends on the specific type of wave.

Light and IR radiation are EM waves, the disturbances are electric and magnetic fields. The electric and magnetic fields are perpendicular to each other and to the direction of propagation, this means that EM waves are transverse waves. The maximum disturbance 
of the wave is called amplitude. Figure 2.1 shows an overview of the EM spectrum ordered according to wavelength, the IR region is emphasized.

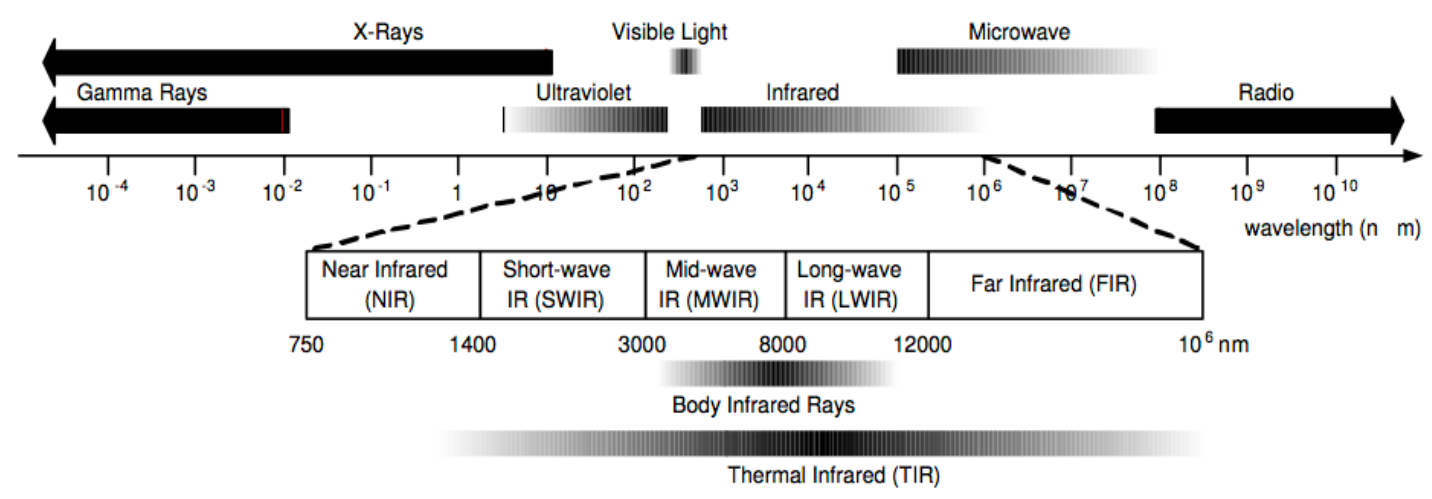

Fig. 2.1 The electromagnetic spectrum and the thermal infrared region [Qi and Diakides 2007].

The IR spectrum roughly covers the wavelength range from $750 \mu \mathrm{m}$ to $106 \mathrm{~nm}$, for IR imaging only a portion of this range is used. Typically the three spectral ranges used in thermography are: the long-wave IR (LWIR), the mid-wave IR (MWIR) and the near-infrared (NIR). Commercial cameras are available for these three ranges. Detector technology is a major restrictive influence on the use of a given IR range for imaging. This is a consequence from the physics of the detector and the transmission properties of the atmosphere.

The most important process for thermography is thermal radiation. The concept of thermal radiation implies that every object at a temperature higher than $0 \mathrm{~K}\left(-273.15{ }^{\circ} \mathrm{C}\right)$ emits $\mathrm{EM}$ radiation. The maximum radiant power that can be emitted by any object depends on the temperature of the object and its material. Blackbody radiation helps explain the radiation 
emitted by all objects. A blackbody is a perfect absorber and emitter of radiation and has three main characteristics: 1) it absorbs every incident radiation, regardless of wavelength and direction, 2) for a given temperature and wavelength, no surface can emit more energy than a blackbody, 3) radiation emitted by a blackbody depends on wavelength, however its radiance does not depend on direction. In nature no blackbody exists and thus a blackbody is often realized by cavities whose walls are kept at constant temperature. The radiation of a real object can be calculated by multiplying the blackbody radiation with the emissivity, $\varepsilon$, value of the real object. Emissivity of an object is the ratio of the amount of radiation emitted from the surface to that emitted by a blackbody at the same temperature. The emissivity value is essential to IR imaging and depends on many parameters. It is of great importance to know the emissivity value of the object under study during IR imaging. In this dissertation we are imaging the human skin, whose emissivity value is 0.98 ; this value tell us that the human skin is close to being a blackbody. In actuality, objects with high emissivity values are called gray bodies.

\subsection{Infrared Camera System}

The main purpose of an IR camera is to convert infrared radiation into a false color visual image. The main components of a camera are the optics, the detector, the cooling or temperature stabilization of the detector, the electronics for signal and image processing, and the user interface with output ports, control ports, and the image display (figure 2.2). 


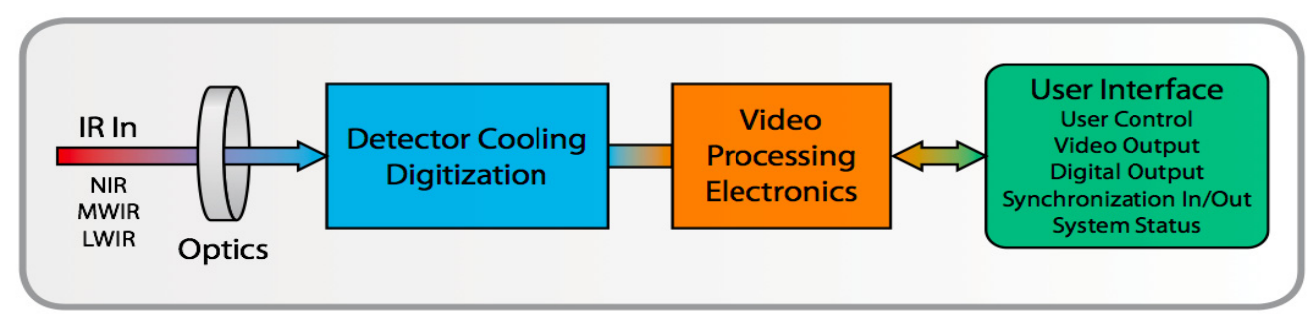

Fig. 2.2 Block diagram of an IR camera system [Courtesy of FLIR Systems IR Handbook]

During the data collection portion of this dissertation, we used the Merlin ${ }^{\mathrm{TM}}$ MWIR camera system shown in Figure 2.3. The following sections of this chapter give a brief overview of the components in Figure 2.2. When pertinent, the corresponding characteristic in the Merlin ${ }^{\mathrm{TM}}$ MWIR camera will be noted.

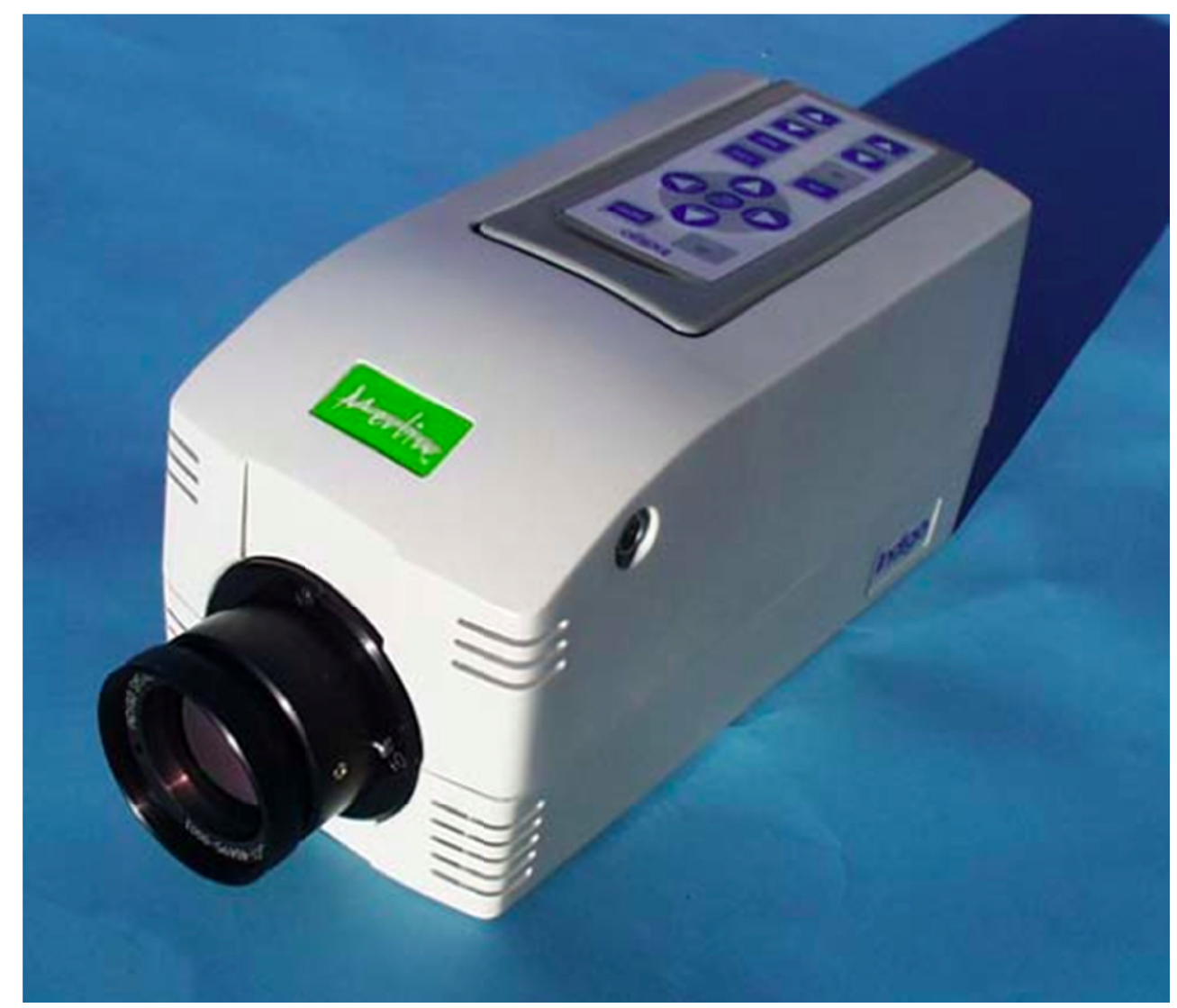

Fig. 2.3 Merlin ${ }^{\mathrm{TM}}$ MWIR camera used for data collection. [Courtesy of FLIR ${ }^{\mathrm{TM}}$ Systems] 


\subsubsection{Camera Design and Image Formation}

Two basic systems exist for thermal imaging: 1) scanning system and 2) staring systems.

In a scanning system an image is generated as a function of time row by row; in a staring system, the image is projected simultaneously onto all pixels of the detector array. The Merlin ${ }^{\mathrm{TM}}$ MWIR consists of a staring array or also known as a focal plane array (FPA).

Figure 2.4 shows the basic components of a scanning system and a staring system.
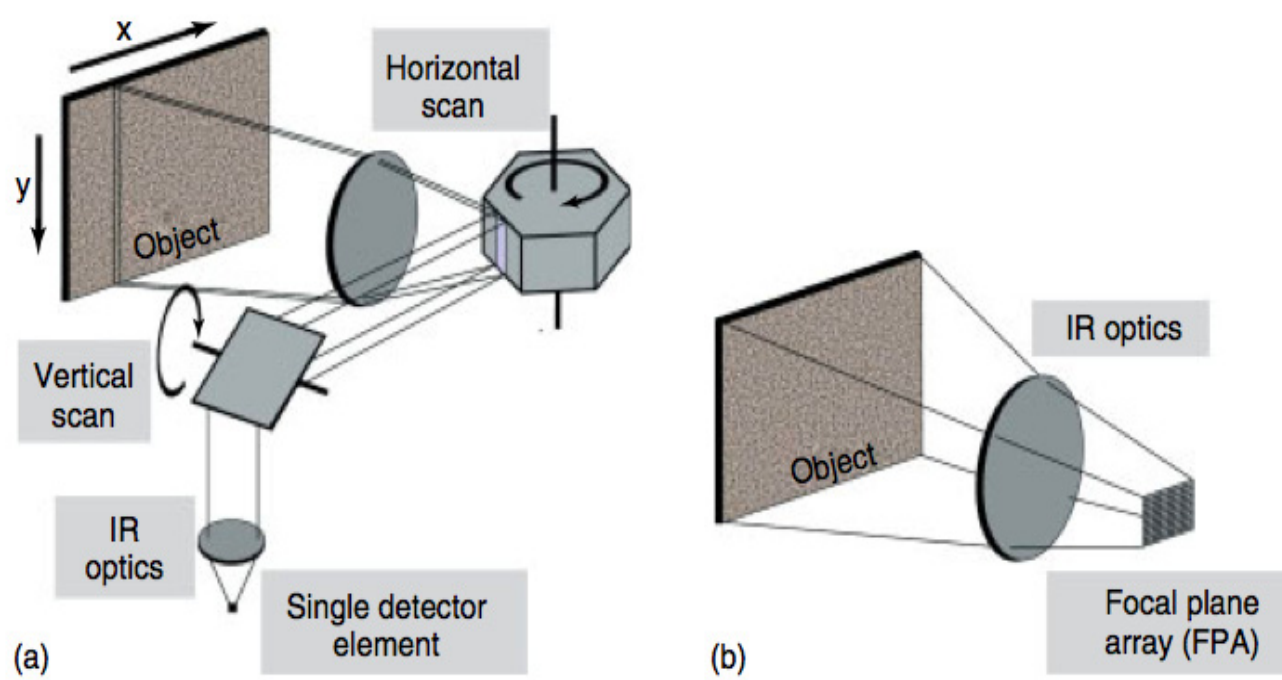

Fig. 2.4 Basic components for (a) scanning system and (b) staring system [Vollmer and Mollman 2010]

\subsubsection{Focal Plane Arrays}

In a focal plane array the detectors are arranged in a matrix of columns and rows. The advantage of a staring system is that the detector array covers the whole field of view (FOV) simultaneously. A focal plane array for IR imaging consists of two parts: the infrared sensor made from a material sensitive to infrared radiation and a readout integrated circuit (ROIC) made from silicon. The ROIC has two functions: 1) it does 
signal readout and 2) contributes to the signal processing with the signal amplification and integration or with multiplexing and analog-to-digital conversion. The Merlin TM MWIR camera consists of an Indium Antimonide (InSb) Focal Plane Array (FPA) built on an Indigo Systems ISC9705 (ROIC) using indium bump technology.

\subsubsection{Non-uniformity Correction}

An FPA is composed of many individual detector elements having different levels of signal responsiveness and offsets. The spread in gain and offset results in a spread of detector signals for the same incident radiant power. If this non-uniformity of the individual detectors becomes too large then the image becomes unrecognizable and the non-uniformity has to be corrected, this is known as non-uniformity correction (NUC). For most commercial cameras the NUC procedure is done during the factory calibration process and the NUC parameters are stored in the camera firmware. The Merlin ${ }^{\mathrm{TM}}$ MWIR camera allows the user to perform one-point and two-point correction in internal and external mode. However is not recommended to do a two-point correction by the user as the process is done using blackbody sources at the factory. Directions to perform a one-point correction are found in the technical user's manual of the Merlin ${ }^{\mathrm{TM}}$ MWIR camera.

\subsubsection{Detector Temperature Stabilization and Detector Cooling}

For optimum operation of detectors in thermal IR imaging systems stabilization of the detector temperature or detector cooling is necessary. During the early stages of IR 
imaging the MWIR range cameras used liquid nitrogen at $77 \mathrm{~K}$ to cool the detector. The FPA in the Merlin TM MWIR camera is cooled to a temperature of approximately $77 \mathrm{~K}$ using a Stirling-cycle linear cryocooler that is thermally coupled to the FPA via a cold finger. The Stirling cooler is a sealed refrigeration unit that uses helium as the working gas. It does not require the user to refill the camera with cryogens such as liquid nitrogen. Helium is used because it will stay in the gaseous phase at $77 \mathrm{~K}$ even at high pressures. The cooler uses a compression step, followed by an expansion to remove heat from the cold finger. The compression and expansion process is repeated at a rapid rate, resulting in a low humming noise. The electronics in the Merlin ${ }^{\mathrm{TM}}$ MWIR camera control the micro-cooler unit and achieves excellent temperature stability. The typical time to cool down the FPA to $77 \mathrm{~K}$ is $7-8$ minutes.

\subsubsection{Optics and Filters}

\subsubsection{Spectral Response}

Most cameras are characterized by a broad spectral response depending on the characteristics of the detectors. The FPA in the Merlin ${ }^{\mathrm{TM}}$ MWIR camera is a $320 \times 256$

matrix of $\mathrm{InSb}$ detectors sensitive to the $1 \mu \mathrm{m}-5.4 \mu \mathrm{m}$ range. The standard camera configuration incorporates a cold filter that restricts the camera's spectral response to the 3.0- 5.0 micron band. Figure 2.5 shows the spectral response for other IR cameras. 


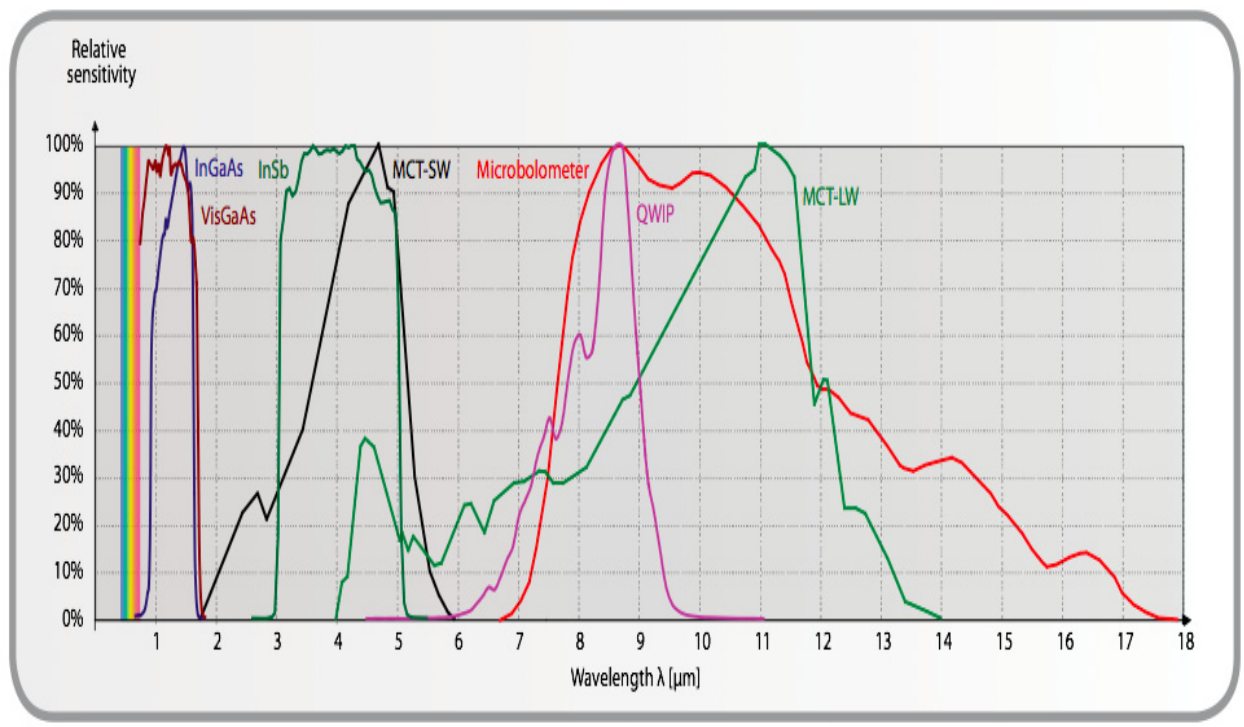

Fig. 2.5 Spectral response for various IR cameras.[Courtesy of FLIR Systems]

\subsubsection{Field of View}

The object field is transformed to an image within the field of view (FOV) of the camera.

The FOV is the angular extent of the observable object field. The camera optics allow a change of the camera FOV. The Merlin ${ }^{\mathrm{TM}}$ MWIR has a $25 \mathrm{~mm}$ lens and this yields a $22 \times 16^{\circ} \mathrm{FOV}$

A brief overview of the main components of an IR system has been given and the corresponding characteristics of the Merlin ${ }^{\mathrm{TM}}$ MWIR camera have been mentioned. Table 2.1 summarizes the Merlin ${ }^{\mathrm{TM}}$ MWIR specifications.

Table 2.1 Technical specifications of the Merlin ${ }^{\text {TM }}$ MWIR

\begin{tabular}{|l|l|}
\hline Thermal sensitivity & $0.025^{\circ} \mathrm{C}\left(0.018^{\circ} \mathrm{C}\right.$ typical $)$ \\
\hline Image frequency & $60 \mathrm{~Hz}$ non interlaced \\
\hline Camera f/\# & 2.5 \\
\hline Focus & Manual \\
\hline Detector Type & Indium Antimonide $(\mathrm{InSb})$ \\
\hline Spectral range & $\begin{array}{l}1.5-5.5 \text { microns }(3-5 \text { microns set by } \\
\text { cold filter })\end{array}$ \\
\hline
\end{tabular}




\begin{tabular}{|c|c|}
\hline Array format & $320 \times 256$ \\
\hline Integration time & $5 \mu \mathrm{s}-16.5 \mathrm{~ms}$ \\
\hline Detector cooling & Integral Stirling \\
\hline Pixel pitch & $30 \times 30 \mu \mathrm{m}$ \\
\hline Temperature measurement & $0-350^{\circ} \mathrm{C}$ \\
\hline Video output & $\begin{array}{l}\text { Analog video @60 Hz } \\
\text { S-video } \\
\text { Digital video } \\
\text { Digital video output: RS-422 }\end{array}$ \\
\hline Weight & 9 lbs. \\
\hline Size $(H \times$ x X L) & $5.5 " \times 5.0 " \times 9.8 "$ \\
\hline Tripod mounting & $1 / 4 " \times 20 "$ w/guide pin notch \\
\hline Remote control options & Button panel \& RS-232 \\
\hline Temperature range & $0^{\circ} \mathrm{C}$ to $+350{ }^{\circ} \mathrm{C}$ \\
\hline Accuracy & $\pm 2{ }^{\circ} \mathrm{C}, \pm 2 \%$ of reading \\
\hline
\end{tabular}

\subsection{Software and Hardware Connection}

The Merlin ${ }^{\circledR}$ MWIR camera communicates with a Microsoft Windows ${ }^{\circledR}$ PC through a standard Ethernet and iPort grabber connection. Figure 2.6 shows a schematic of the connection configuration between the Merlin ${ }^{\circledR}$ MWIR camera and the computer.

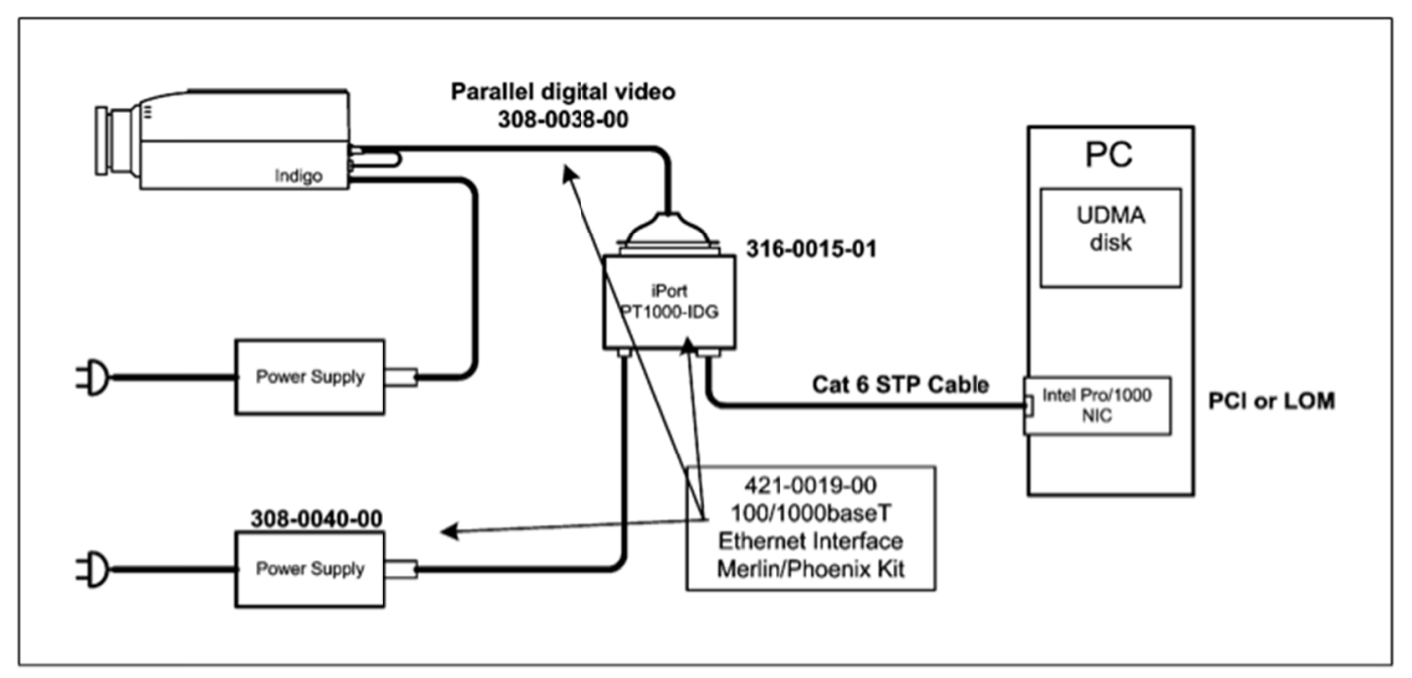

Fig 2.6 Connection configuration between the Merlin ${ }^{\circledR}$ MWIR camera and PC. [Courtesy of FLIR Systems] 
The specifications for the Windows ${ }^{\circledR}$ PC are the following: Intel ${ }^{\circledR}$ Core ${ }^{\mathrm{TM}} 2$ Quad CPU with a speed of $2.40 \mathrm{GHz}, 3.0 \mathrm{~GB}$ RAM, Microsoft Windows XP Professional v. 2000 SP3. FLIR Systems provide their proprietary software ThermaCAM ${ }^{\mathrm{TM}}$ Researcher V2.8 SR-1 for recording or viewing thermal infrared images and video. This software was used for data collection.

After the thermal images were obtained they ware processed using MATLAB ${ }^{\circledR}$ R2009b and the FSLView tool in the FMRIB Software Library [Woolrich et al. 2009, Smith et al. 2004] for image registration. The data processing segment of this study was done on a MacBook Pro running Mac OS X version 10.6.8, with a 2.16 GHz Intel Core Duo, and 2GB SDRAM.

\subsection{Experimental Setup}

For the purpose of this study we collected thermal infrared images from 13 different subjects. The Merlin ${ }^{\mathrm{TM}}$ MWIR camera was placed on a tripod and a chair was placed in front of the camera at a distance of one meter from the subject. The recording of the thermal infrared images was done in a room with an average room temperature of $23^{\circ} \mathrm{C}$. Each subject was asked to sit straight in front of the thermal infrared camera and asked to look straight into the lens and a snapshot of their frontal view was taken. This process was repeated at least three more times in different days and times of the day to take into consideration subtle variations that may occur. Figure 2.7 shows the camera set up for data collection during one experiment aimed to extract jugular and temporal vasculature 
features.

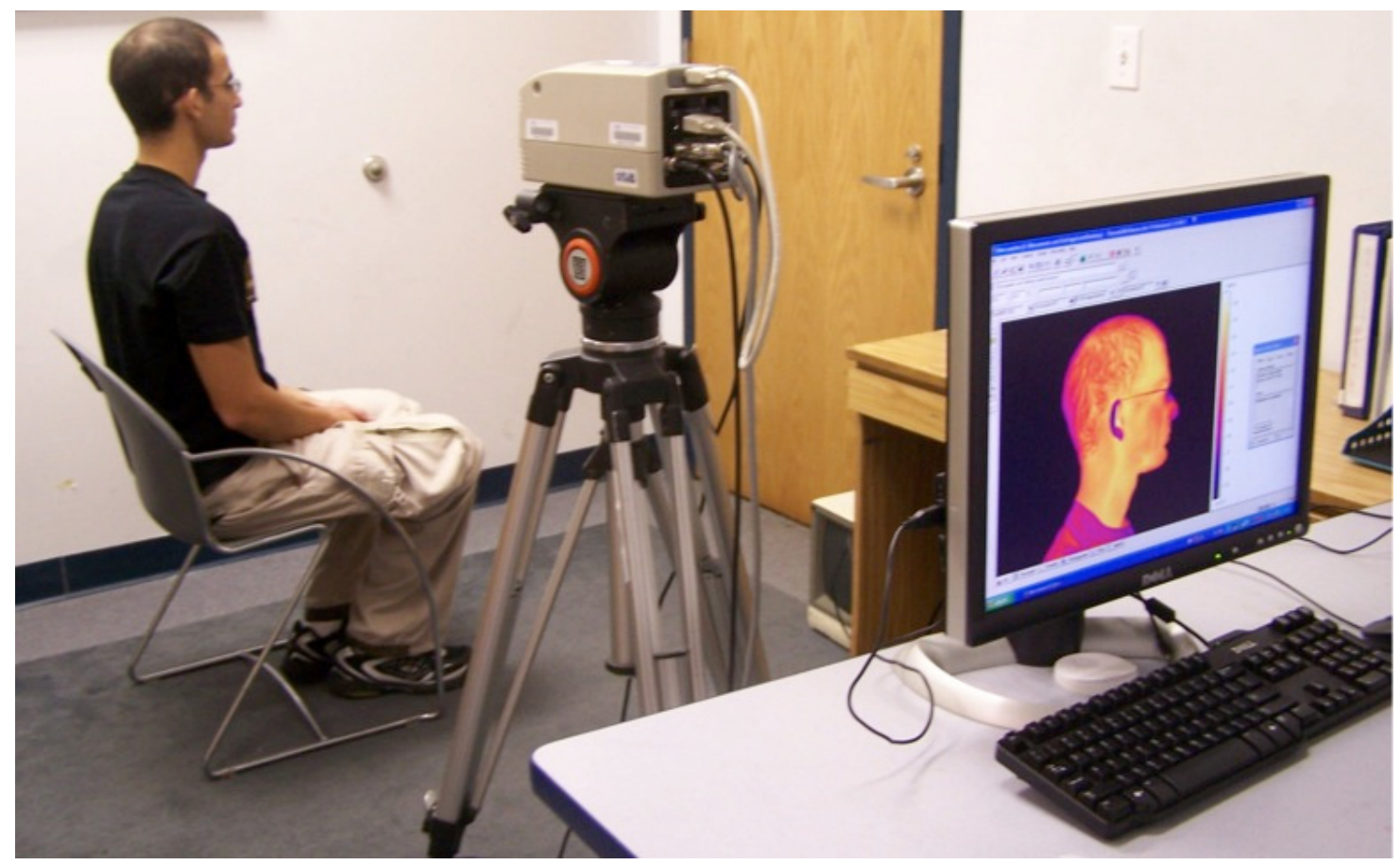

Fig. 2.7 Merlin ${ }^{\mathrm{TM}}$ MWIR camera set up for data collection in the Center for Advanced Technology and Education 


\section{CHAPTER 3}

Generating Facial Thermal Signatures Using Thermal Infrared Images

\subsection{Introduction}

Thermal infrared imaging currently has uses that go beyond the military field, for example, during the swine flu outbreak of 2009 the government and airport personnel at various international airports used thermal infrared cameras to detect possible cases of swine flu [Hidalgo, 2009]. Firefighters and other first responders have used portable thermal imagers to see through smoke, find the origin of a fire, and for the search and rescue of lost individuals. In the medical area, thermal imaging is used to monitor some physiological changes in human beings [Agarwal et al., 2007 and 2008] and other warm-blooded animals [Reese,2006; Church et al. 2009]. Different studies using thermal infrared imaging have been done to detect spontaneous emotional facial expressions [Zheng 2006; Wang et al., 2008; Hernandez et al. 2007], skin tumors [Mital and Scott, 2007], frustration [Pavlidis et al., 2007], temperature increase on the ear and cheek after using a cellular phone [Straume et al., 2005], as well as to recognize faces [Friedrich and Yesburun, 2002, Bebis et al. 2006]. The list of applications continues to expand as the technology to create thermal imaging devices continues to improve and the systems become more affordable. The work in this dissertation focuses on thermal imaging of the

human skin to generate facial signatures, resembling fingerprints, for future implementation of a biometric system. 
Skin forms the largest organ of the human body, skin accounts for about 16 percent of a person's weight. It performs many vital roles as both a barrier and as a regulating influence between the outside world and the controlled environment within our bodies. Internal body temperature is controlled through several processes, including the combined actions of sweat production and the rate of blood flowing through the network of blood vessels within the skin. Skin temperature can be measured and visualized using a thermal infrared camera with a reasonable sensitivity. Facial skin temperature is closely related to the underlying blood vessels; thus by obtaining a thermal map of the human face we can also extract the pattern of the blood vessels just below the skin.

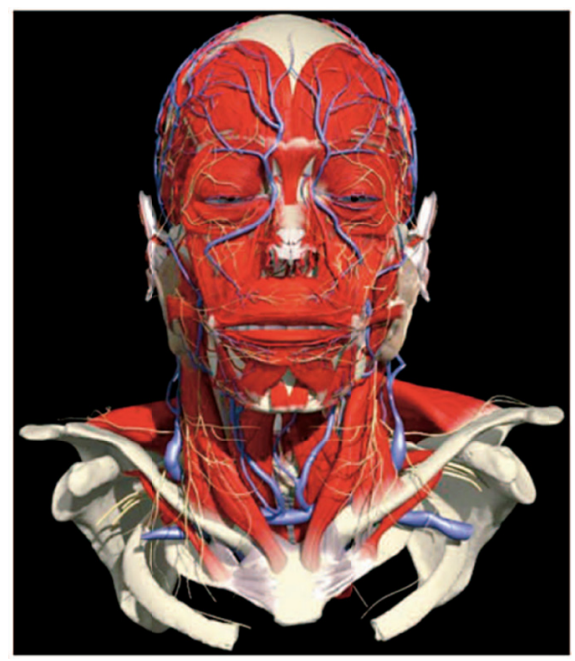

Fig. 3.1 Human facial vascular network [Moxham et al., 2002]

Studies presented in [Buddharaju et al. 2007 ; Zhu et al. 2008] provide an algorithm for the extraction of the human face thermal signature network and the supraorbital vessels in the MWIR range. Figure 3.1 illustrates the arteries and veins in the human face. In our previous work [Guzman et al., 2010] we replicated the study in [Buddharaju et al., 2007]. 
In this dissertation, we present a modified approach to detect thermal signatures and introduce the generation of thermal facial templates. Together the thermal signatures and templates are used for subject authentication or verification.

\subsection{Image Processing}

In this section we process the thermal images from 13 subjects, Figure 3.2 shows an unprocessed thermal image from one subject. The process to record the images was explained in chapter 2 . The feature extraction process which is graphically represented in Figure 3.3 consists of three main steps: image registration, thermal signature extraction, and generation of template.

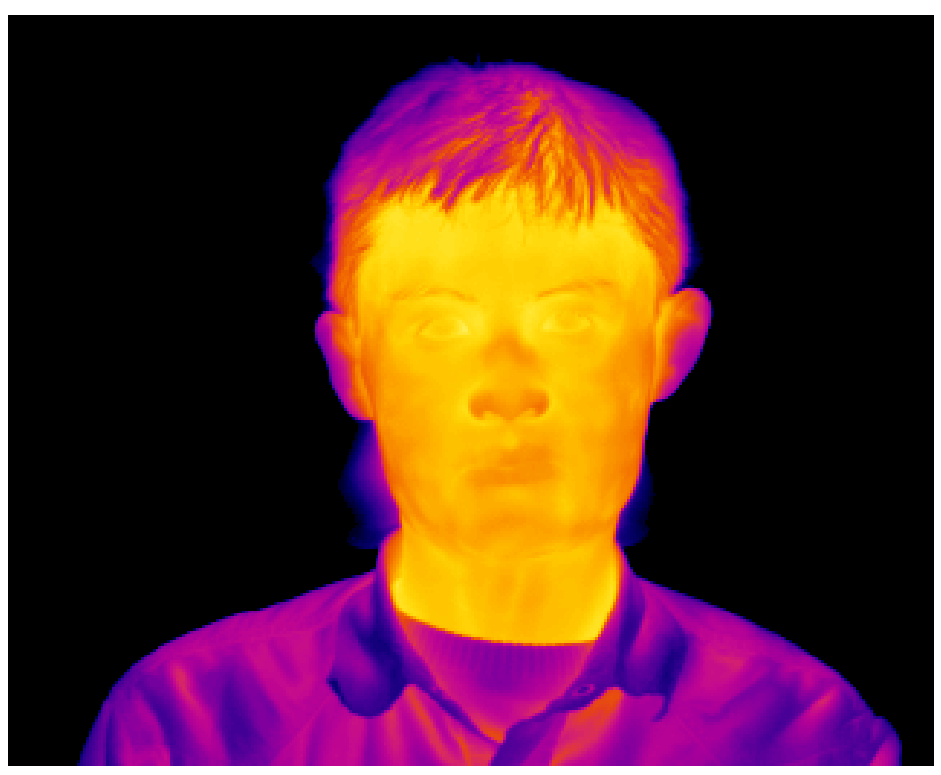

Fig. 3.2 Thermal infrared image of a volunteer 

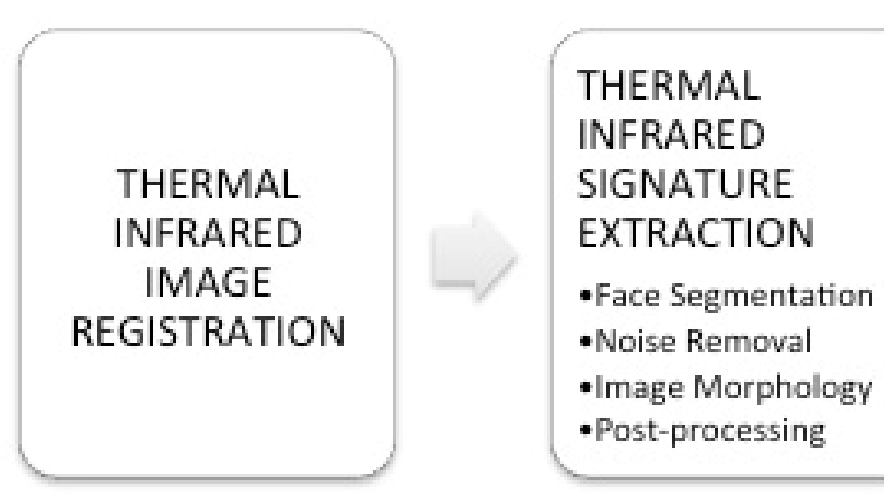

GENERATING

THERMAL FACIAL

SIGNATURE

TEMPLATE

Fig. 3.3 The three main steps to generate thermal facial signatures and templates

\subsubsection{Image Registration}

In this part of our work we first perform intra-subject image registration of the thermal images. The intra-subject image registration process was achieved using the Linear Image Registration Tool (FLIRT), designed by the functional MRI of the Brain (FMRIB) Analysis Group at the University of Oxford, assuming the rigid body model option for 2D image registration. FLIRT has been shown to be significantly faster and accurate in image registration as compared to other techniques such as simulated annealing of the genetic algorithms for Magnetic Resonance Imaging (MRI) applications. However, the use of FLIRT for registering thermal images as presented in this dissertation has not been addressed before to the best of our knowledge.

We used four thermal images from each subject, one was chosen as the reference image and the rest were registered to the reference image. The thermal image of the subject is taken at different times therefore there are slight lateral and vertical shifts in the position of the subject relative to the camera's position. The FLIRT registration of these two images 
greatly depends on the parameters chosen for the registration task. The various parameters that need to be addressed are: cost function, degrees of freedom (DOF), and interpolation. The FLIRT algorithm aims at reducing an intensity-based cost function in order to register the images. These cost functions include the normalized correlation, mutual information and correlation ratio. The choice of the cost function depends on the nature of the image to be registered in terms of size and gray scale, relative to other images. Also, since both the input and the reference images in this case are of the same modality, a within-modality cost function has to be employed to obtain better results. It was found that for the thermal images under consideration, the 'mutual information' cost function gave us the best registration results among the different options employed. The quality of the registration was inspected through manual visualization by overlaying the original and the registered images.

The degrees of freedom (DOF) describe the search limits for the registration algorithm. A complete registration of two images requires four DOF. Two of the four DOF are for the two dimensions of the image, the third one for rotation, and the fourth one for scaling. The FLIRT algorithm is employed thus using 4 DOF to achieve a complete registration between two images.

The interpolation step of the process is only used for the final transformation, not in the registration calculations. The interpolation step is used in order to obtain a final output image after the interpolation step in case the registration needs to supplement missing information from the surrounding pixels. Various types of interpolations can be employed 
but since the images are fairly aligned with only slight shifts in position, a simple nearest neighbor interpolation was found to be sufficient.

Also, since FLIRT has been primarily designed for medical image processing applications, it only accepts the reference images and the input images in medical image format. For this purpose, each image is first converted to the Neuroimaging Informatics Technology Initiative (NIfTI) format for image registration. For this purpose an in-house developed script is used to convert the images to NIfTI and vice-versa.

An important task for the project was the automation of the registration process. The automation process is required for the registration of the large number of images in the dataset as well as for the registration of new input images. Since the entire processing was carried out in MATLAB ${ }^{\circledR}$, a shell script was created to interact with and control the FLIRT algorithm through the MATLAB ${ }^{\circledR}$ environment. The shell script has the role of importing the input and reference images, setting the parameters for the FLIRT and exporting the registered image for further processing.

Figures 3.4-3.6 show an example where the subject position changed causing a vertical shift in the image, note the shift at the bottom of figure 3.6. Registering the thermal images to a reference thermal image of the subject facilitates the process of creating a thermal signature. 


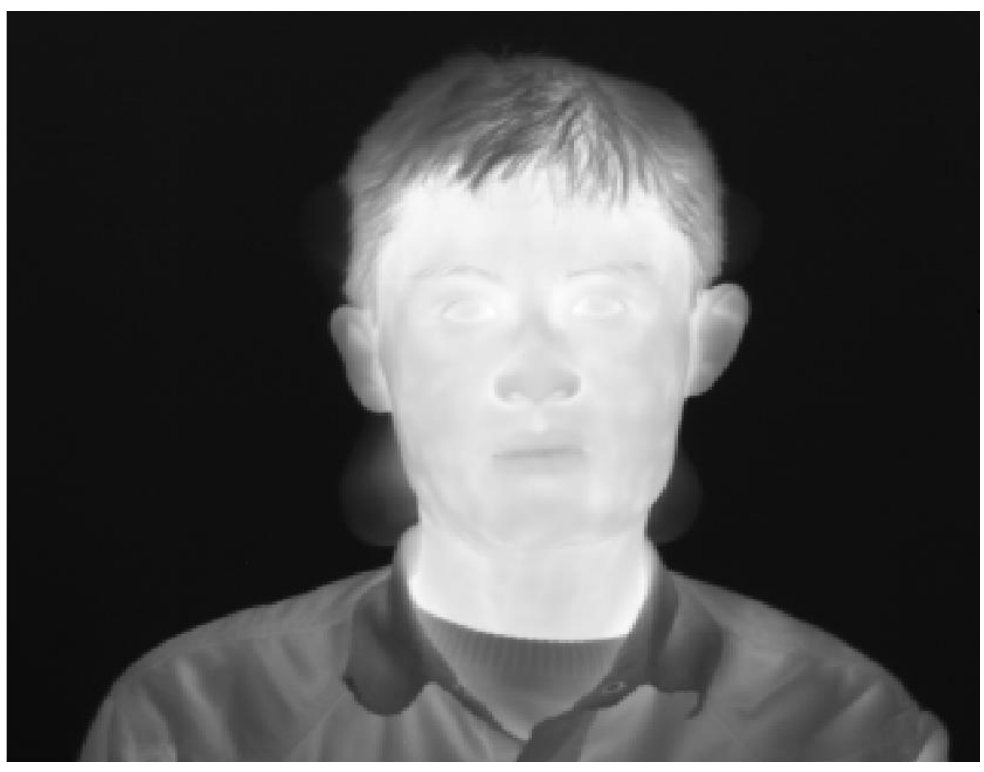

Fig. 3.4 Reference thermal image

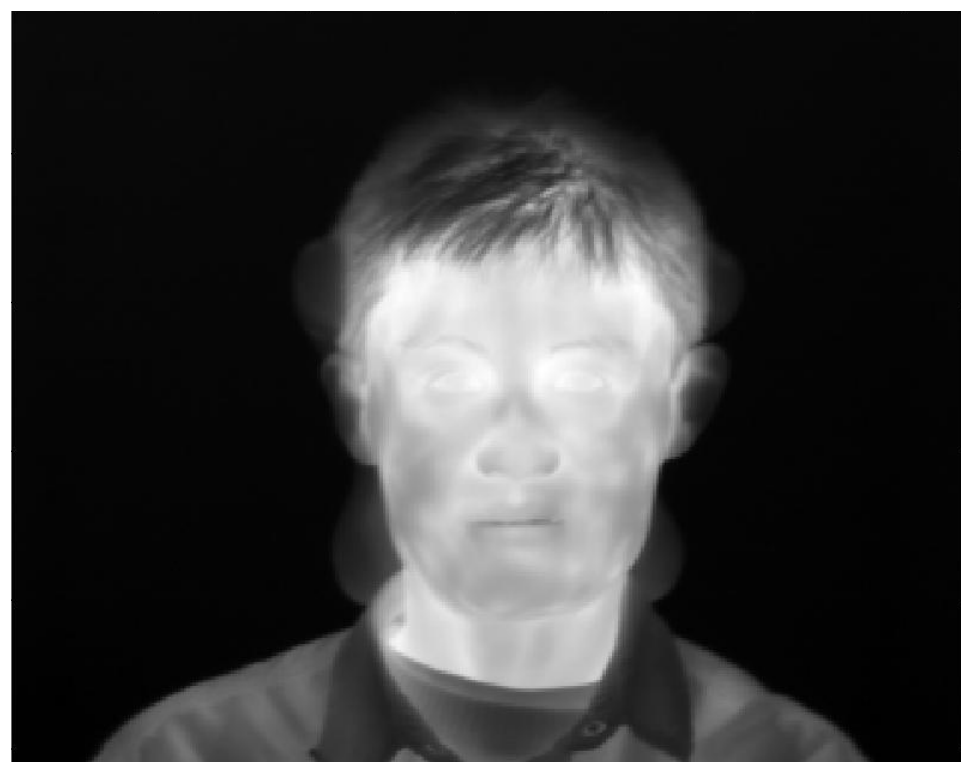

Fig. 3.5 Non-reference thermal image 


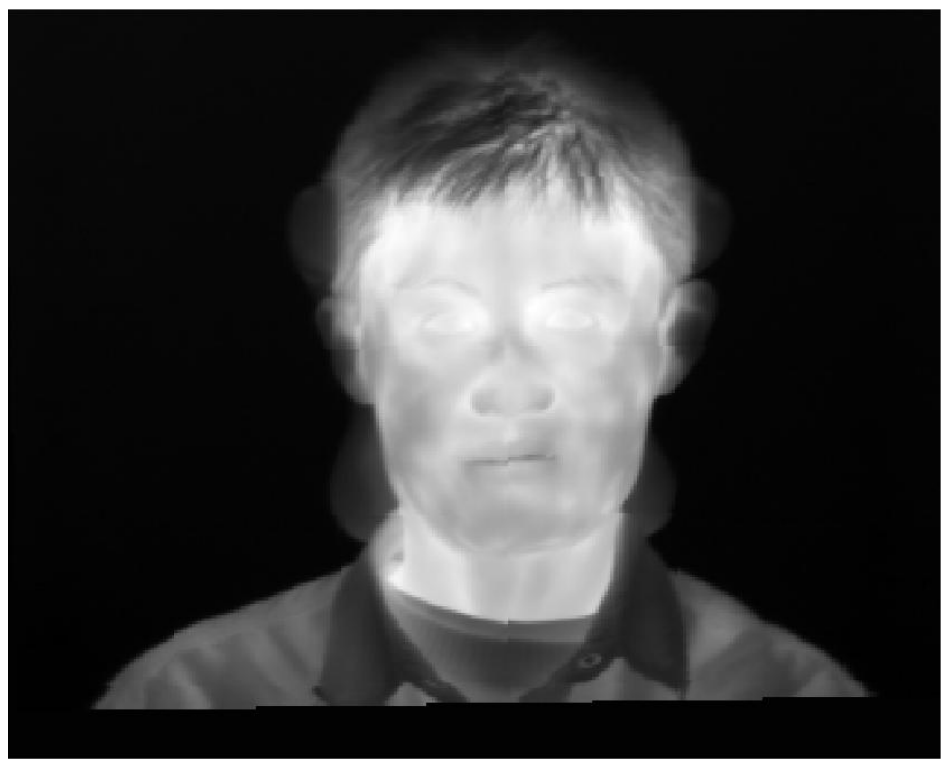

Fig. 3.6 Registration of Figure 3.4 using FLIRT

\subsubsection{Thermal Signature Extraction}

After registering the thermal images for each subject we proceeded to extract the thermal signature in each image. The thermal signature extraction process has four main sections: face segmentation, noise removal, image morphology, and post-processing.

\subsubsection{Face Segmentation}

In this step the face of the subject was segmented from the rest of the image. The segmentation process was achieved by implementing the technique of localizing region based active contours in which typical region-based active contour energies are localized in order to handle images with non-homogeneous foregrounds and backgrounds [Lankton, Tannenbaum; 2008]. Localized region-based contours operate by growing an initial contour selected by the user roughly around the face to segment the face region. The facial region segmented in this study does not take into consideration the neck area of the subject. This is 
achieved by localizing the contouring algorithm to a neighborhood around the point of interest with a localization radius of 5 pixels.

Let $\Psi=\{\mathrm{x} \mid \Phi(\mathrm{x})=0\}$ be a closed contour of interest. The interior of the closed contour $\Psi$ is expressed in terms of the smoothed approximation of the signed distance function given as

$$
H \phi(x)=\left\{\begin{array}{lc}
1, & \Phi(\mathrm{x})<-\varepsilon \\
0, & \Phi(\mathrm{x})>\varepsilon \\
\frac{1}{2}\left\{1+\frac{\Phi}{\varepsilon}+\frac{1}{\pi} \sin \frac{\pi \Phi(\mathrm{x})}{\varepsilon}\right\}, & \text { otherwise }
\end{array}\right.
$$

where $\phi(x)$ is a smoothed initial contour and $[-\varepsilon, \varepsilon]$ represents the boundary of the Heaviside function. In reference to Eq.1 the exterior of the closed contour is given as $\{1-H \phi(\mathrm{x})\}$. In order to model the energies of the interior and exterior of the contour for segmentation purposes the well-known Yezzi energy is used [Hua and Yezzi 2003]. The Yezzi energy defines a dual-front active contour, which is widely used for segmentation purposes in cases where the solution may fall in a local minima and yield poor results.

The algorithm operates by first dilating the user selected initial contour to create a potential localized region $R$ for finding the optimal segmentation. Thus,

$$
R=\phi \oplus \mathrm{S}
$$

where $S$ is the spherical structuring element of the localization radius (i.e. 5 pixels) and $\oplus$ is the dilation operator. 
The algorithm proceeds by evolving the inner and outer boundaries of $R$ to reach minima where the inner and outer boundary contours intersect after applying a single iteration of the algorithm called the dual-front active contour region growing technique. The newly formed intersection acts as a new initialization and the process is repeated until the Yezzi energy function is reduced. Figures 3.7 and 3.8 show the original thermal image and the result of segmentation, respectively.

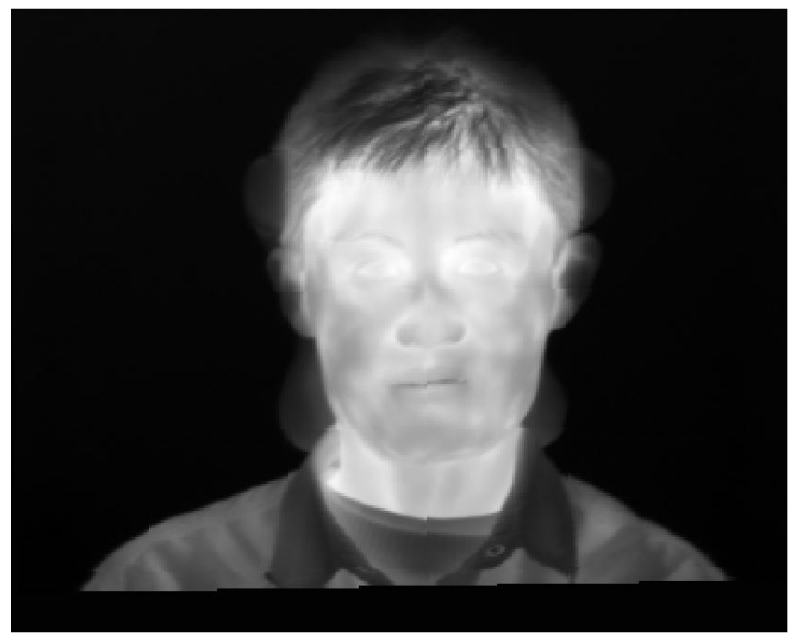

Fig. 3.7 Registered thermal infrared image

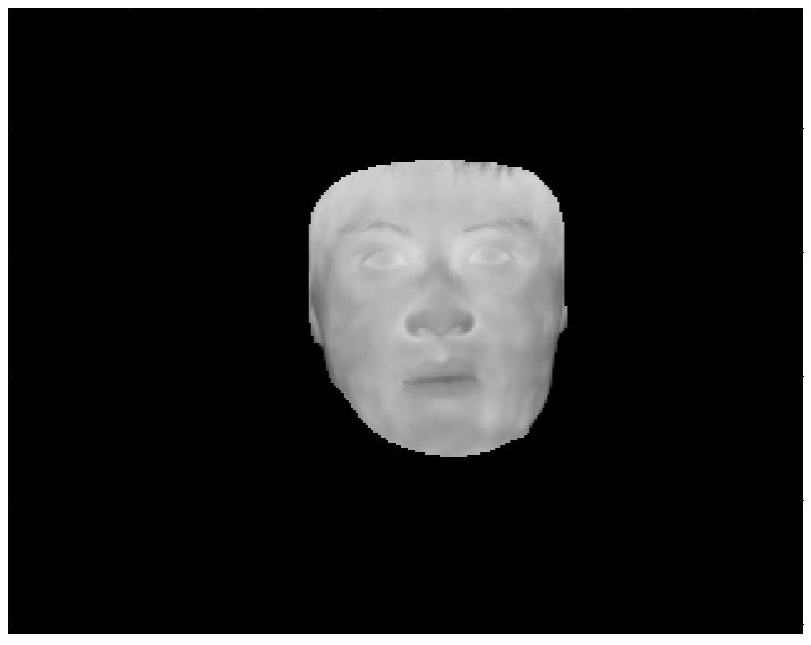

Fig. 3.8 Final face segmentation 


\subsubsection{Noise Removal}

After the face was segmented from the rest of the thermal image we proceeded to remove unwanted noise in order to enhance the image for further processing.

A standard Perona-Malik anisotropic diffusion filter [Perona, Malik 1990] is first applied to the entire the image with the segmented face. For image processing and computer vision applications, anisotropic diffusion is a technique used to reduce the image noise without removing significant parts of the image content, typically edges, lines or other details that are important for the interpretation of the image.

The significance of the anisotropic diffusion filter in this particular application is to reduce spurious and speckle noise effects seen in the images and to enhance the edge information for extracting the thermal signature. For the diffusion filter a $2 \mathrm{D}$ network structure of 8 neighboring nodes is considered for diffusion conduction. The considered neighbors are the north, south, east, west, northeast, northwest, southeast and southwest. The conduction coefficient function used for the filter applied on the thermal images aims to privilege edges over wider regions in order to enhance regions of high thermal activity associated with the thermal signature. Thus, the conduction coefficient function used for the application is given by Eq. 3

$$
C(x, y, t)=\frac{1}{1+\left(\frac{\|\nabla I\|}{K}\right)^{2}}
$$

where $\nabla I$ is calculated for the 8 directions and $\mathrm{K}$ is the gradient modulus threshold that controls the conduction. 
The anisotropic diffusion filtering is an iterative process, where a relatively simple set of computations is used to compute each successive image in the family, this process is continued until a sufficient degree of smoothing is obtained, the filtering process here uses 10 iterations. The choice of the number of iterations was empirical in nature with studies aimed to reduce spurious noise while at the same time maintaining the overall information in the image. The requirement that the image does not lose the details of the structural elements of the face, such as eyes, lips, and nose determines the upper boundary for the iterative process.

\subsubsection{Image Morphology}

Morphological operators are based onset theory, the Minkowsky operators and DeMorgan's law. Image morphology is a way of analyzing images based on shapes. In this study we assume that the blood vessels are a tubule like structure running along the length of the face. The operators used in this experiment are opening and top-hat segmentation.

The effect of an opening operation is to preserve foreground regions that have a similar shape to the structuring element or that can completely contain the structuring element, while eliminating all other regions of foreground pixels. The opening of an image can be mathematically described by:

$$
I_{\text {open }}=(I \Theta S) \oplus \mathrm{S}
$$


where $I$ and $I_{\text {open }}$ are the face segmented image and the opened image respectively; $\Theta$ and $\oplus$ are the morphological erosion and dilation operators.

The top-hat segmentation has two versions, for our purpose we use the version known as white top-hat segmentation as this process enhances the bright objects in the image; this operation is defined as the difference between the input image and its opening. The selection of the top-hat segmentation is based on the fact that we desire to segment the regions associated with those of higher intensity, which demark the facial thermal signature. The result of this step is to enhance the maxima in the image. The top-hat segmented image $I_{\text {top }}$ is given by:

$$
I_{\text {top }}=I-I_{\text {open }}
$$

\subsubsection{Signature Segmentation Post-Processing}

After obtaining the maxima of the image we skeletonize the maxima. Skeletonization is a process for reducing foreground regions in an image to a skeletal remnant that largely preserves the extent and connectivity of the original region while throwing away most of the original foreground pixels.

The skeletonization approach used in this study is a homotopic skeletonization process whereby a skeleton is generated by image morphing using a series of structural thinning elements from the Golay alphabet [Meihua et al., 2009].

Morphological thinning is defined as a hit-or-miss transformation which is essentially a binary template matching where a series of templates $\mathrm{L}_{1}$ through $\mathrm{L}_{8}$ are searched 
throughout the image. A positive search results in a 1 and a miss results in a 0 . This is given mathematically as,

$$
\mathrm{I}_{\text {skel }}=\mathrm{I}_{\text {top }} \mid\left(\mathrm{I}_{\text {top }} \underline{\widehat{x}} L_{i}\right)
$$

where $\underline{\hat{x}}$ is the hit-or-miss operator and $L_{i}$ is the set of structuring elements $\mathrm{L}_{1}$ through $\mathrm{L}_{8}$. The first two structuring elements used for the skeletonization process are shown in Eq.7.

$$
L_{1}=\left[\begin{array}{lll}
1 & 1 & 1 \\
0 & 1 & 0 \\
0 & 0 & 0
\end{array}\right] \quad L_{2}=\left[\begin{array}{lll}
0 & 1 & 0 \\
0 & 1 & 1 \\
0 & 0 & 0
\end{array}\right]
$$

The rest of the 6 structuring elements can be obtained by rotating both the masks $\mathrm{L}_{1}$ and $\mathrm{L}_{2}$ by $90^{\circ}, 180^{\circ}$ and $270^{\circ}$. In Figure 3.9 we show the final result of the process outlined in subsections 3.2.1-3.2.2.

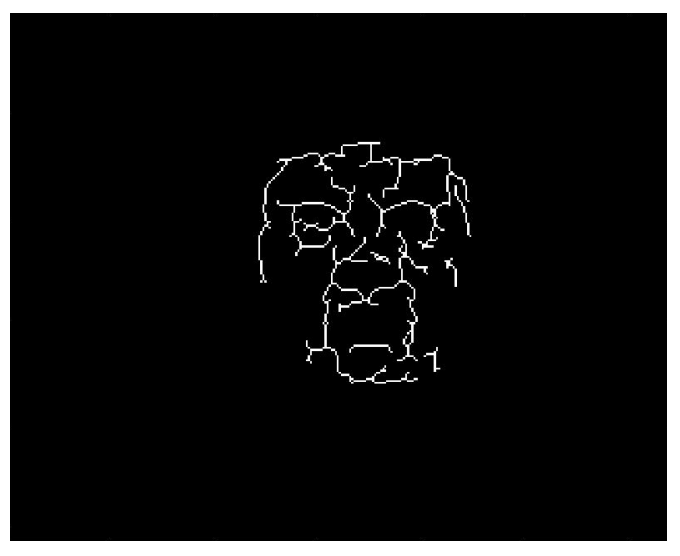

Fig. 3.9 Result of skeletonizing a facial thermal image

\subsubsection{Generation of Thermal Signature Template}

Thermal signatures in an individual vary slightly from day to day basis due to various reasons [Jones et al., 2002]. These reasons include: 
1) Exercise: Exercise just prior to image capture may result in variations in the thermal signatures due to increase bodily activities.

2) Environmental Temperature: The environmental temperature from which the subject arrives to the image capture room may result in change in thermal signatures in extreme cases, therefore is necessary for the subject to be allowed to acclimatize to the temperature of the room where the images are taken.

3) Weight: In some cases a weight gain or loss may results in a difference in the thermal signatures, it is well known that fat tissue is a good insulator thus affecting the surface temperature of the skin.

4) Health of the subject: The health of the subject is a parameter that could affect the thermal signatures. A fever that results in an increased body temperature may change the thermal signature.

5) Temperature of the imaging room: The temperature of the imaging room can have effects on the thermal signature. Please note that in this study we have made efforts to maintain a constant temperature on the imaging room and allowed the subject to acclimatize to the room temperature.

Taking into consideration the various factors that may change the thermal signature on daily basis we propose a technique of determining a thermal signature template, which aims to preserve only the important characteristics in a person's thermal signature over time. 
The generation of a thermal template consists of taking the extracted thermal signatures for each subject and adding them together. The resulting image is a composite of four thermal signature extractions, each one slightly different from the other. By adding the thermal signatures our goal is to keep the features that are present in all the images as the dominant features that define the subject's signature. We then apply an anisotropic diffusion filter to the summation of the thermal signatures in order to fuse the predominant features.

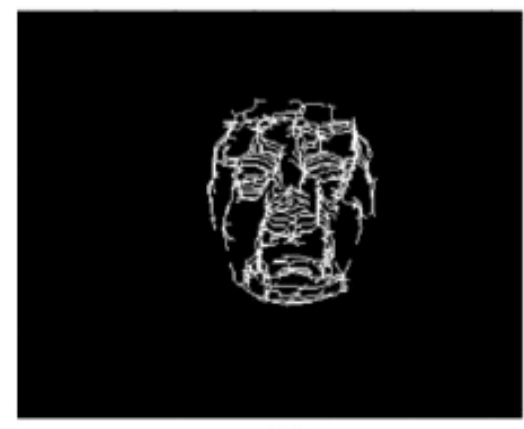

(a)

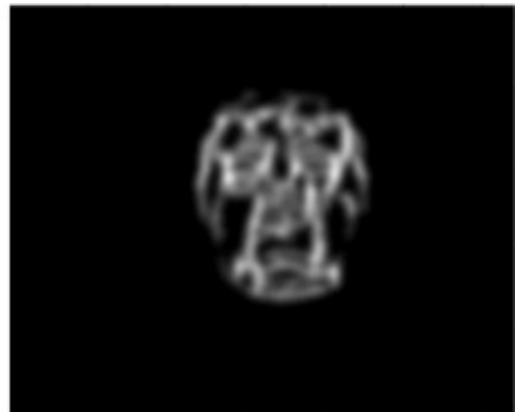

(b)

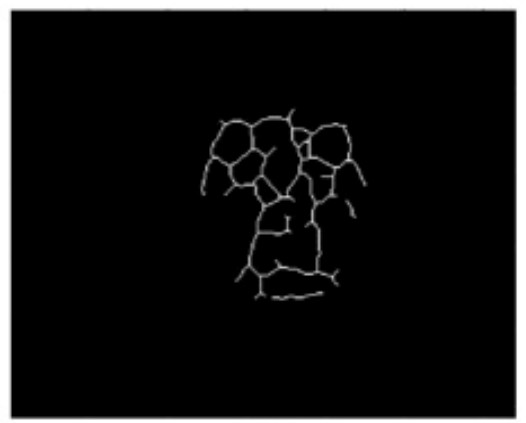

(c)

Fig. 3.10 Process used to generate the signature thermal template: (a) addition of four single signatures, (b) anisotropic diffusion, (c) skeletonization

The skeletonization of the obtained diffused image results in a single thermal template for that person, figure $3.10 \mathrm{a}-\mathrm{c}$ shows the results for the generation of thermal templates. For 
all future testing purposes a template database is created against which a newly acquired signature will be tested. The testing process is explained in chapter 4 .

\subsection{Examples of Thermal Facial Signatures and Templates}

The process outlined in sections 3.2.2 and 3.2.3 is applied to every thermal image obtained from each subject. Figures 3.11-3.16 show the results of performing the described process on three subjects, the images clearly show a distinct thermal template for each individual.

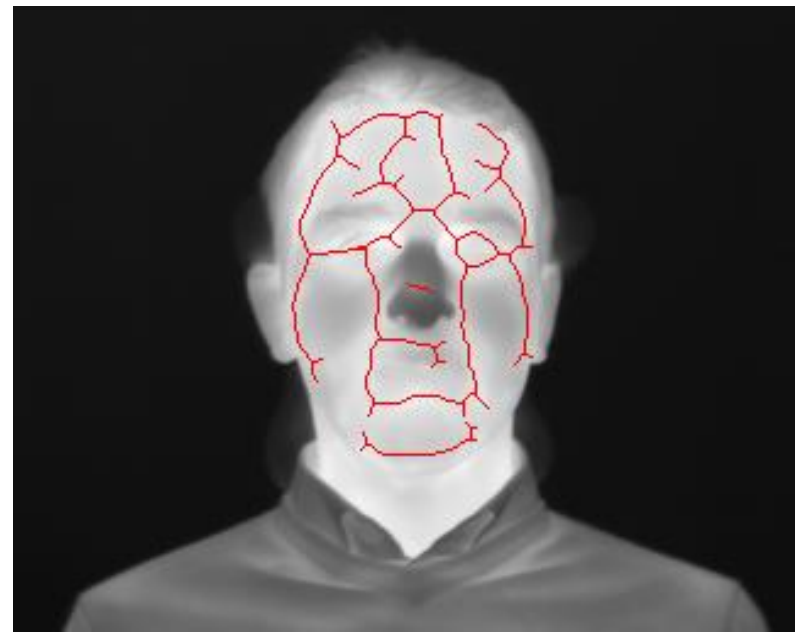

Fig. 3.11 Overlay of thermal signature template on a subject's thermal infrared image

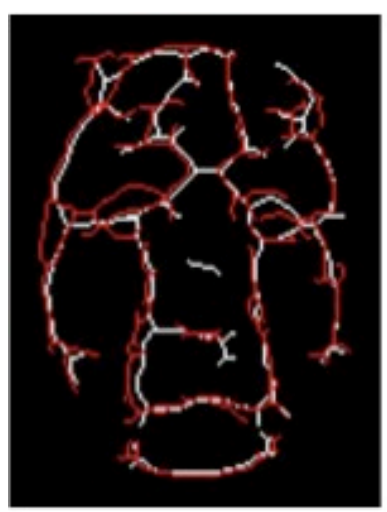

(a)

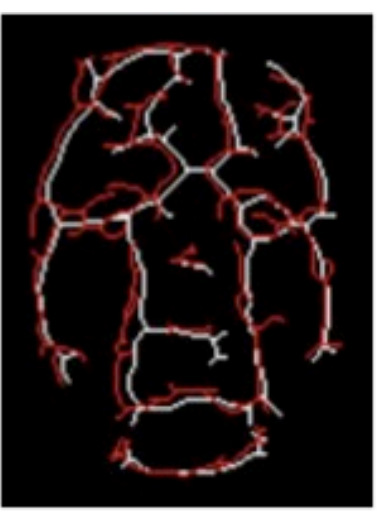

(b) 


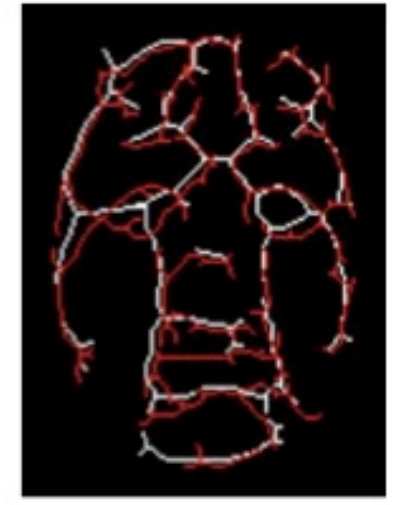

(c)

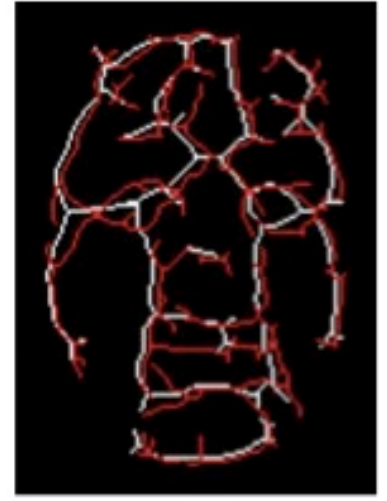

(d)

Fig. 3.12 Overlay of thermal signature template (white) on four single thermal signatures (red) belonging to subject in figure 3.11

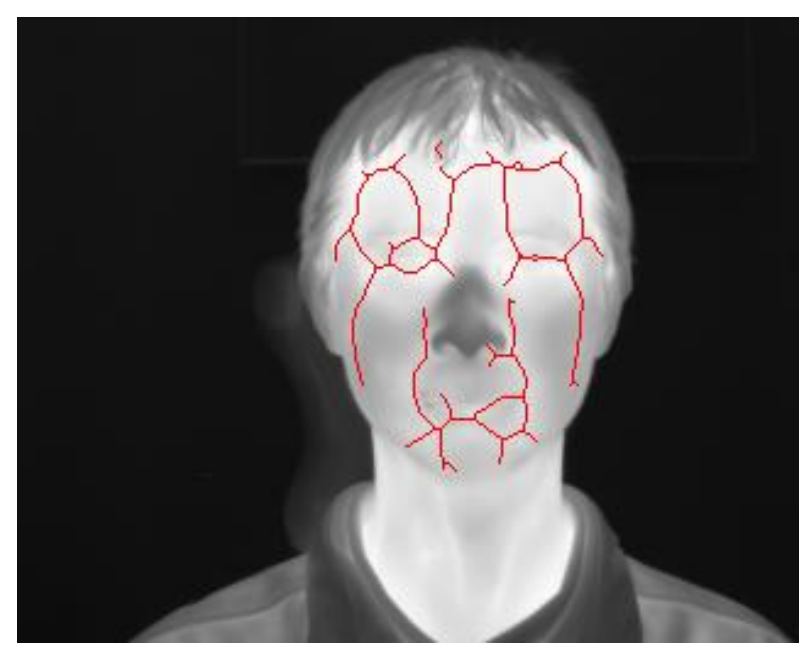

Fig. 3.13 Overlay of signature thermal signature on a subject's thermal infrared image

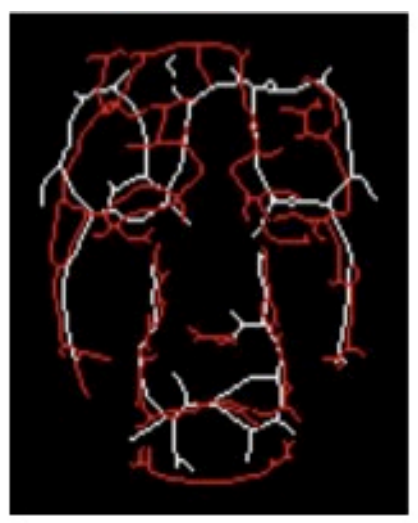

(a)

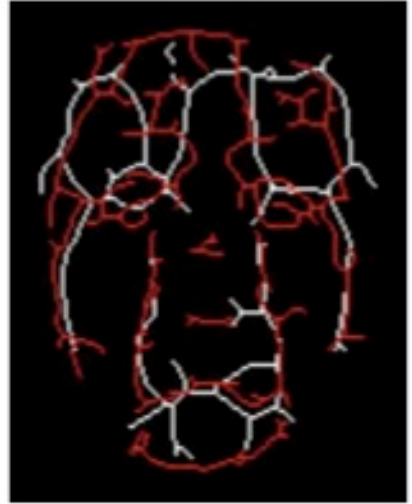

(b) 


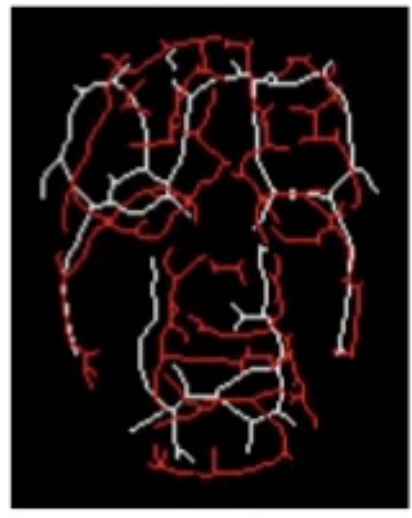

(c)

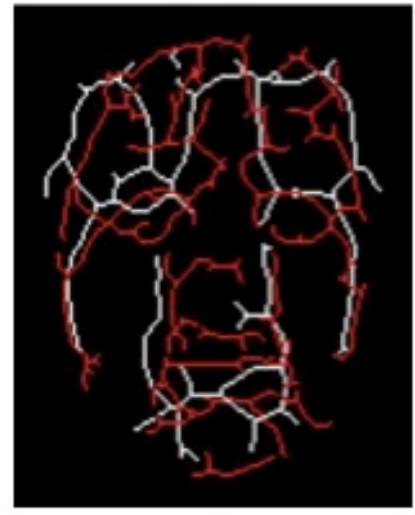

(d)

Fig. 3.14 Overlay of thermal signature template (white) on four single thermal signatures (red) belonging to subject in figure 3.13

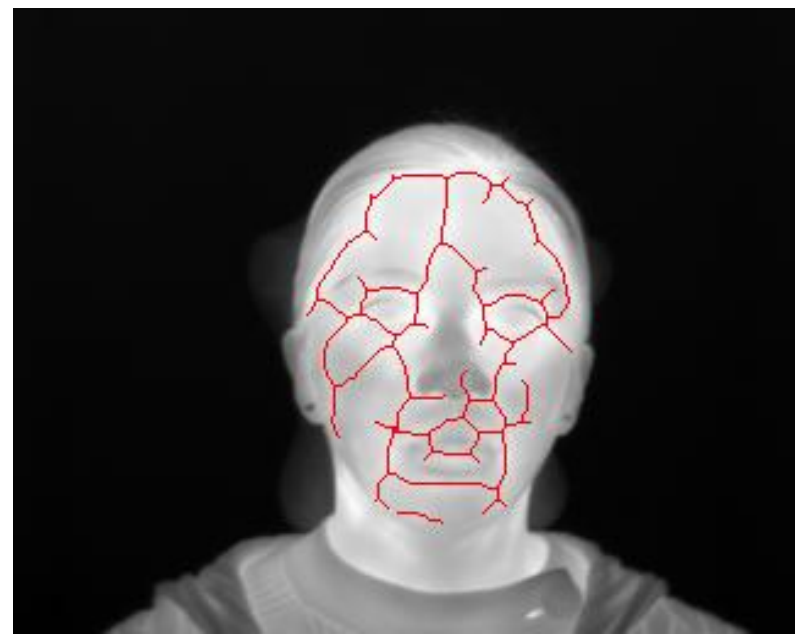

Fig. 3.15 Overlay of signature thermal signature on a subject's thermal image

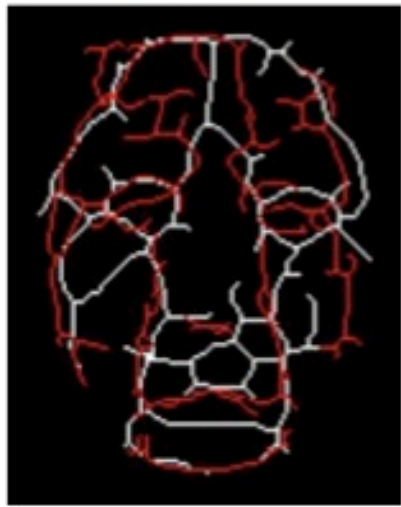

(a)

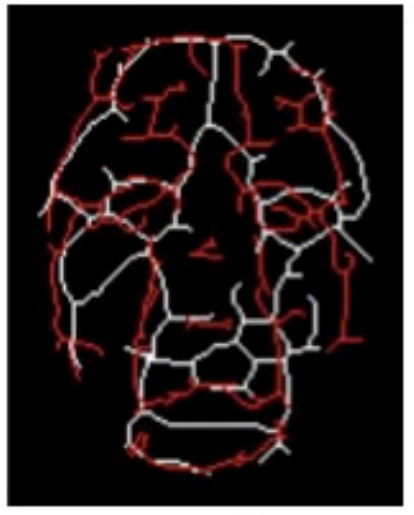

(b) 


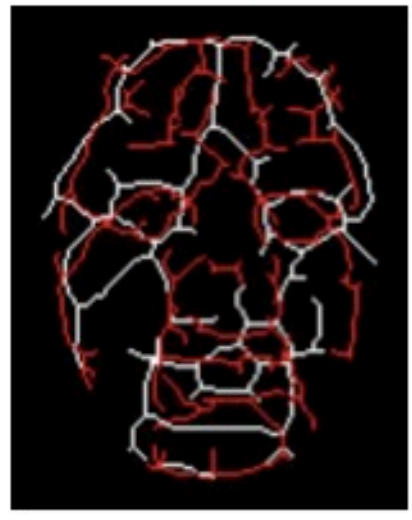

(c)

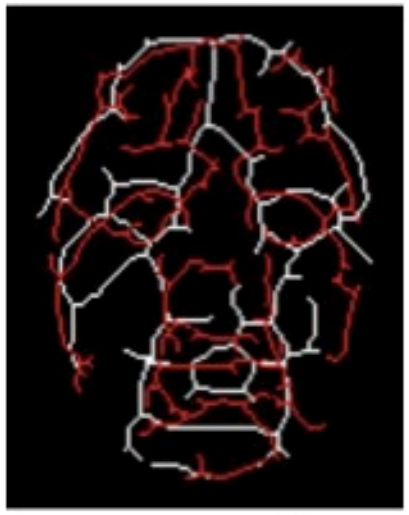

(d)

Fig. 3.16 Overlay of signature thermal signature (red) on individual thermal template (white) of subject in figure 3.15

The presented examples show that thermal infrared images allow us to extract thermal facial features through a set of integrated image processing techniques. The results also show that the thermal facial signature among individuals is unique and there is little change on its structure when the images are taken at different times, and through the generation of a signature thermal signature we can observe that there are thermal features that remain constant. These features that remain constant will allow us to match the thermal signature to a specific individual. The implementation will then extend to a robust biometric system. 


\section{CHAPTER 4}

Similarity Measures

\subsection{Introduction to Biometrics}

\subsubsection{Introduction}

There has always been a need to identify individual biometrics; however the means of identification have changed drastically as populations have grown and as individuals have become more geographically mobile. Biometric technologies have emerged as tools to help identification in a global and mobile society. The term biometrics in the information technology field refers to the distinctive use of identifiers or anatomical and behavioral characteristics for authentication or recognition purposes.

Many identification systems comprehend three elements: 1) attribute identifiers (e.g. Social Security Number, driver's license number, account number), 2) biographical identifiers (e.g. address, profession, education, marital status), and 3) biometric identifiers (fingerprint, voice, gait). Traditionally the use of attribute and biographical identifiers to verify a person's identity was sufficient but with population growth and individuals becoming more geographically mobile the need for a robust and flexible identity management system has led to the proliferation of biometric systems. Current biometric identification technology promises to strengthen the relationship between attribute and biographical identifiers. It is rather easy for an individual to falsify attribute and biographical attributes, however biometric identifiers are generally understood as more 
secure because it is assumed that a body identifier (fingerprint, iris, face, voice) is difficult to falsify.

\subsubsection{Biometric Recognition}

A biometric identifier is the measurement of an anatomical or behavioral characteristic taken from a living human body. Business and government institutions often rely on answers to questions about a person's identity to either provide or deny a service. These and other institutions need reliable ways or methods for identification. Because biometric identifiers cannot be easily displaced, forged, or shared, they are considered more reliable than traditional methods. The objectives of biometric recognition are: user convenience, better accountability, and better security. The fingerprint is the most widely known biometric for identity identification, the technology, algorithms, and modalities in this area are well explained in [Maltoni et al., 2009]. Advancements in other technologies and modality areas have given way to the use of other traits as means of biometric authentication, these traits include: face, iris, hand geometry, voice, signature, handwriting, hand vein, DNA, ear shape, fingernail bed, palmprint, keystroke dynamics, and retina geometry [Bhattacharyya et al. 2009, Delac and Grgic 2004]. In [Ratha and Govindaraju 2008, Jain et al. 2008, Wayman et al. 2005] a comprehensive and detailed review is given on the current state of the listed biometric traits, algorithms and systems.

\subsubsection{Biometric Systems}

A biometric system consists of four modules: sensor module, feature extraction module, 
matching module, and the decision making module. Figure 4.1 presents a visual representation of the four modules.
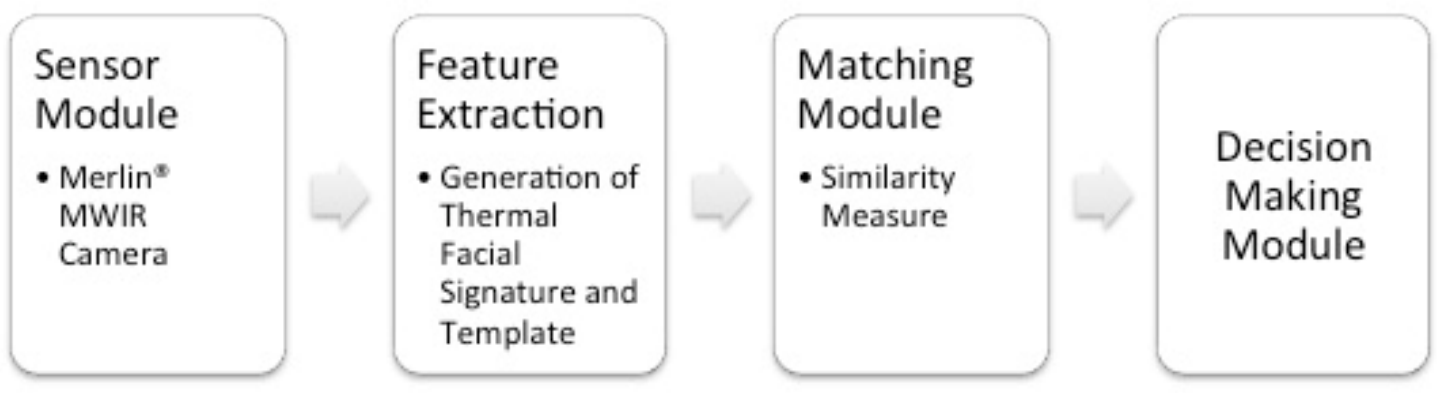

Fig. 4.1 Basic modules of a simple biometric system

In the sensor module, biometric data is acquired; in this study we acquired thermal infrared images of 13 volunteer subjects using the Merlin ${ }^{\mathrm{TM}}$ MWIR camera, the process was described in Chapter 2. The feature extraction module is where the acquired data is processed to extract feature vectors; in our study we extracted thermal facial signatures and created templates, the process was described in chapter 3. The matching module is where the feature vectors are compared against those in a template; this module is explained in section 4.3 of this chapter. First, we introduce the concept of similarity measures in section 4.2.

\subsection{Similarity Measures}

\subsubsection{Introduction}

Similarity measures are basically functions that quantify the degree to which two or more objects are similar to each other. The concept of similarity is very important in almost every scientific field. In the field of mathematics studies of congruence make use of 
geometric methods to assess similarity in studies of congruence. Fuzzy set theory has also developed its own measures of similarity, with applications on management, medicine and meteorology. In molecular biology it is of great importance to measure the sequence similarity of protein pairs [Ashby and Ennis 2007]. In image processing similarity measures have been used for image storage and retrieval in databases [Jain et al. 1995, Aksoy and Haralick 2001]. In this study we present a different approach to match a thermal facial signature with its corresponding thermal facial template stored in the database. We propose the use of a similarity measure based on the well-known Euclidean distance to match a thermal facial signature to its corresponding thermal facial template in the database.

\subsubsection{General definition of Similarity Measures}

Similarity measures are used in different domains and as a consequence their terminology varies (coefficients of association, resemblance or matching), and some authors might propose, independently, a similarity measure with different names. However, some common properties are shared. In [Lesot and Rifqi 2009] three definitions of similarity measure are presented, we summarize these in the next subsections.

\subsubsection{Similarity Measure: Definition I}

Definition1: Defining $\chi$ as the data space or universe, the similarity measure $\mathrm{S}$ can be defined as a function $\chi \times \chi \rightarrow \mathbb{R}$ that verifies the following properties:

- Positivity: $\forall x, y \in \mathrm{X}, S(x, y) \geq 0$. 
- Symmetry: $\forall x, y \in \mathrm{X}, S(x, y)=S(y, x)$.

- Maximality: $\forall x, y \in \mathrm{X}, S(x, x) \geq S(x, y)$.

The first property establishes that a similarity measure is always positive for all $x$ and $y$ in $\chi$. The second property establishes symmetry when comparing $x$ and $y$, this means that the similarity measures have the same order whether one compares ' $x$ to $y$ ' or ' $y$ to $x$ '. The third property tells us that the comparison of ' $x$ to $x$ ' (i.e. identity) should have a higher or equal value to the similarity value of the comparison ' $x$ to $y$ '.

\subsubsection{Similarity Measure: Definition II}

The properties mentioned in section 4.2.2.1 are sometimes relaxed thus leading to a more general definition. In 1977 Amos Tversky proposed the rejection of the of the symmetry constraint. He argued on the directional nature of the similarity relation of the from ' $x$ is like $y^{\prime}$ [Lesot and Rifqi 2009]. Indeed this is true if for example $x$ and $y$ are two images where $x$ has more features than $y$, then the symmetry property for similarity measures does not hold. It is important to introduce some notations in order to present the second definition of similarity measures. Given two objects $x=\left(x_{1}, \ldots, x_{\mathrm{p}}\right)$ and $y=\left(y_{1}, \ldots, y_{\mathrm{p}}\right)$ both belonging to $\{0,1\} \mathrm{p}$, let $\mathrm{X}=\left\{\mathrm{i} x_{\mathrm{i}}=1\right\}$ and $\mathrm{Y}=\left\{\mathrm{i} y_{\mathrm{i}}=1\right\}$ be the set of attributes present in objects $x$ and $y$ respectively. Similarity measures are often expressed as functions of four quantities associated to the object couple $(x, y)$.

- a, denotes the number of attributes common to both objects: $|\mathrm{X} \cap \mathrm{Y}|$

- $\quad b$, denotes the number of attributes present in $\mathrm{x}$ but not in $\mathrm{y}:|\mathrm{X}-\mathrm{Y}|$ 
- $\quad \mathrm{c}$, denotes the number of attributes present in $\mathrm{y}$ but not in $\mathrm{x}:|\mathrm{Y}-\mathrm{X}|$

- $\quad \mathrm{d}$, denotes the number of attributes in neither $\mathrm{x}$ nor $\mathrm{y}:|\bar{X} \cap \bar{Y}|$

These characteristics help define similarity measures that depend only on characteristics that are either present in $x$ or in $y$, but are independent of the attributes absent in both objects. These kind of similarity measures are referred to as Type 1, Table 4.1 lists some Type 1 similarity measures.

Table 4.1 Type 1 similarity measures [Lesot and Rifqi 2009]

\begin{tabular}{lcc}
\hline Similarity measure & Notation & Definition \\
\hline Jaccard (1908) & $S_{\text {Jac }}$ & $\frac{a}{a+b+c}$ \\
Dice (1945) & $S_{\text {Dic }}$ & $\frac{2 a}{2 a+b+c}$ \\
Sorensen (1948) & $S_{\text {Sor }}$ & $\frac{4 a}{4 a+b+c}$ \\
Anderberg (1973) & $S_{A n d}$ & $\frac{8 a}{8 a+b+c}$ \\
Sneath and Sokal 2 (1973) & $S_{S S 2}$ & $\frac{a}{a+2(b+c)}$ \\
Ochiai (1957) & $S_{O c h}$ & $\frac{a}{\sqrt{a+b} \sqrt{a+c}}$ \\
Kulczynski 2 (1927) & $S_{K u 2}$ & $\frac{1}{2}\left(\frac{a}{a+b}+\frac{a}{a+c}\right)$ \\
Rifqi et al. (2000) & $S_{F D}$ & $\frac{F_{F D}(\phi)-F_{F D}\left(\frac{\pi}{2}\right)}{F_{F D}(0)-F_{F D}\left(\frac{\pi}{2}\right)}$
\end{tabular}

It can be seen from the table that the measures follow a pattern and are defined as fractions of a linear combination of $a, b, c$. They only differ through a multiplicative coefficient $k$ in the quotient $k a$ and in the sum $k a+b+c$, the multiplicative coefficient $k$ gives a variation to the importance of the common and distinctive characteristics of the two objects. 
Tversky generalized the similarity measures shown in Table 4.1 using his proposed contrast model [Lesot and Rifqui 2009] leading to the second definition of similarity measures.

Definition 2: Given two positive real numbers $\alpha$ and $\beta$ a Tversky similarity measure is of the form:

$$
\forall(x, y) \in \mathrm{X}^{2}, S_{T_{v e}}(x, y)=\frac{a}{a+\alpha \cdot b+\beta \cdot c}
$$

This contrast model defines similarity measures in a more general manner as it doesn't impose the symmetry property. The similarity measures in Table 4.1 can be obtained through $\alpha=\beta=1 / k=2^{-n}$.

A second type of similarity measures exists, these take into consideration all four quantities $(a, b, c, d)$ derived from the objects. Table 4.2 lists some examples of type 2 similarity measures.

Table 4.2 Type 2 similarity measures [Lesot and Rifqi 2009]

\begin{tabular}{lcc}
\hline Similarity measure & Notation & Definition \\
\hline Rogers and Tanimoto (1960) & $S_{R T}$ & $\frac{a+d}{a+2(b+c)+d}$ \\
Sokal and Michener (1958) & $S_{S M}$ & $\frac{a+d}{a+b+c+d}$ \\
Sokal and Sneath 1 (1963) & $S_{S S 1}$ & $\frac{a+d}{a+\frac{1}{2}(b+c)+d}$ \\
Russel and Rao (1940) & $S_{R R}$ & $\frac{a}{a+b+c+d}$ \\
Yule and Kendall (1950) & $S_{Y u Q}$ & $\frac{a d}{a d+b c}$ \\
& $S_{Y u Y}$ & $\frac{\sqrt{a d}}{\sqrt{a d}+\sqrt{b c}}$ \\
\hline
\end{tabular}


The major difference between type 1 and type 2 similarities is that for type 2 similarity measures the size of the universe influences the similarity. The reader is referred to [Lesot and Rifqi 2009] for a more detailed explanation on type 2 similarities and to [Choi et al. 2010] for a comprehensive list of similarity and distance measures.

\subsubsection{Similarity Measure: Definition III}

In some applications (e.g. image and document retrieval) the user is interested only on a list of objects that is similar to his request, ignoring the similarity score of each object.

It has been pointed out by [Santini and Jain 1999] that the relevant information is contained in the ranking created by the similarity values and not the values directly. In cases in which the ranking is more important than the actual similarity value the choice of two or more similarity measures is of little interest if they lead to the same ranking. In [Lesot and Rifqi 2009] they present studies that have studied the notion of order equivalence, they conclude by defining two measures as equivalent if the measures induce the same ranking, this leads to the third definition of similarity measures.

Definition 3: Two similarity measures $S_{1}$ and $S_{2}$ are equivalent if and only if $\forall x, y, z, t \in \mathrm{X}^{4}, S_{1}(x, y)<S_{1}(z, t) \Leftrightarrow S_{2}(x, y)<S_{2}(z, t)$.

Similarity measures are often applied to binary data and numerical data. The similarity measures presented in sections 4.2.3 and 4.2.4 deal with these two topics respectively.

\subsubsection{Similarity Measures on Binary Data}

The similarity definitions presented in section 4.2.2.1 - 4.2.2.3 are used in binary data. 
The binary feature vector is one of the most common representations of patterns, measuring similarity and distance measures are of high importance in problems such as classification and clustering. Binary similarity measures have been applied in biology, ethnology, taxonomy, image retrieval, geology, chemistry, and ecology [Choi et al., 2010]. The application of similarity measures in these fields is beyond the scope of this work however it was necessary to introduce the similarity definitions as they can be applied in numerical data or data represented as real vectors.

\subsubsection{Similarity Measures on Numerical Data}

Section 4.2.2 explained the similarity measures for binary data and presented in Table 4.1 and Table 4.2 some existing similarity measures. In this section similarity measures for numerical data are explained. The data space can be written as $\mathrm{X}=\square^{p}$ where $p$ is the number of characteristics. The main difference with binary data is the fact that the attributes take their values from a continuous domain, and not from a discrete one. With numerical data the position of two data cannot be characterized by their intersection, set differences, or the intersection of their complementary sets. The information is reduced and depends on a singular quantity that is expressed as a distance or a scalar product between two vectors. For numerical data two types of similarity measures can be defined, similarity measures deduced from dissimilarity and measures based in dot product. In the following section we will turn our focus to similarity measures based on dissimilarity, more specifically on distance based similarities. 


\subsubsection{Similarity Measures Based in Dissimilarity}

One of the classic definitions of similarity consists in deriving a measure from a dissimilarity measure through a decreasing function; which is similar to formulate it from a distance function. In many applications, e.g. stereo matching, not all points from object $x$ have a corresponding point in object $y$, due to occlusion and noise. Most often the two point sets differ in size so a one-to-one correspondence does not exist between all the points. In this case a dissimilarity measure is often recommended. The distances that are often used to compare numerical data are the $L_{\mathrm{p}}$ distances or also known as the Minkowski family. Table 4.3 lists the $L_{\mathrm{p}}$ distances.

Table 4.3 Distance measures $L_{\mathrm{p}}$ [Lesot and Rifqi 2009]

\begin{tabular}{lc}
\hline Name & \multicolumn{1}{c}{ Distance } \\
\hline Euclidean & $d(x, y)=\sqrt{\sum_{i=1}^{p}\left(x_{i}-y_{i}\right)^{2}}$ \\
Weighted Euclidean & $d(x, y)=\sqrt{\sum_{i=1}^{p} \alpha_{i}\left(x_{i}-y_{i}\right)^{2}}$ \\
Mahalanobis & $d(x, y)=\sqrt{(x-y)^{t} \Sigma^{-1}(x-y)}$ \\
Minkowski & $d_{\gamma}(x, y)=\left(\sum_{i=1}^{p}\left|x_{i}-y_{i}\right|^{\gamma}\right)^{\frac{1}{\gamma}}$ \\
\hline
\end{tabular}

The most often used $L_{\mathrm{p}}$ distance is the Euclidean distance $\left(L_{2}\right)$. In section 4.3 we introduce the distance-based similarity measure used for matching thermal infrared signatures to a thermal infrared template in the database. 


\subsection{Distance-Based Similarity Measure for Matching Thermal Signatures and}

\section{Templates}

\subsubsection{Introduction}

Matching and dissimilarity-based measures differ mostly in emphasis and applications. In brief, matching techniques are developed mostly for the recognition of objects under several conditions of distortion whereas similarity measures are used in applications such as image or document retrieval, for example a query image is a partial model of the user's desires and the user looks for images similar to the query image in the database. In our study we make use of similarity measures because we are attempting to find a thermal infrared template similar to the query thermal infrared signature. As it was shown in chapter 3 the thermal infrared template is composed from features present in four thermal infrared signatures, for simplicity from now on we refer only to template and signature as it is understood that they thermal infrared due to the modality used to acquire the images.

\subsubsection{Distance-Based Similarity Measure}

The distance-based similarity measure used in this study is based on the well-known Euclidean metric. In [Candocia and Adjouadi 1997] the authors established a matching strategy to evaluate and confirm stereo matching, we use their stereo vision concept and modify their algorithm for matching thermal facial signatures and templates. In [Candocia and Adjouadi 1997] the authors use stereo image pairs and match features by dividing the images into smaller windows, however in this study we apply the similarity measure to 
two different images and do not create smaller windows.

Given a signature, $A$ (non-reference image), and a template, $B$ (reference image), the similarity measure between $A$ and $B$ is denoted by $S(A \rightarrow B)$. The similarity measure is then computed by Eq. (4.1)

$$
S(A \rightarrow B)=\sum_{i=1}^{h} \frac{P}{\left(D_{i}+1\right)}
$$

where $P$ is the weight associated in matching a feature and is given by Eq. (4.2)

$$
P=\frac{1}{\min \left(N_{A}, N_{B}\right)}
$$

where $N_{\mathrm{A}}$ and $N_{\mathrm{B}}$ are the number of features i.e. white pixels in $A$ and $B$ respectively. Parameter $h$ is the minimum number of feature points found in either $A$ or $B$, i.e. $h=\min \left(N_{\mathrm{A}}, N_{B}\right)$ and finally $D_{i}$ is the minimum Euclidean distance between the $i$ th feature point in $B$ and its closest feature point in $A$. In computing $D_{\mathrm{i}}$ the distance to all features in $A$ to those in $B$ are computed, thus creating a vector of $h$ Euclidean distances for every feature point. The two features that correspond to the minimum distance are then matched, this process continues until all $h$ features are considered.

The authors in [Candocia and Malek 1997] point out that the form in Eq. (4.1) is preferred to other forms such as $1 /\left(D_{\mathrm{i}}+1\right)^{2}$ or $e^{-D}{ }_{i}$, however they fail to provide evidence for their choice. We provide a brief explanation for the reason that lead us to keep Eq. (4.1) in its simple form. The simple form is initially devised to quantify shape distance differences between potential feature matches, where $D_{\mathrm{i}}$ is the Euclidean distance between the feature 
points (pixels) being matched. The simple form is chosen such that this distance difference is inversely proportional to the similarity measure, i.e., the bigger the distance difference, the smaller the similarity measure. Beyond this, it is important to note that Eq. (4.1) takes into consideration that in real world images, small differences will always be there even under the most controlled environments (due to the common effects of noise and distortion), and thus such small differences are made to contribute to the evaluation of the similarity measure.

The following example illustrates that form $(4.1), 1 /\left(D_{\mathrm{i}}+1\right)^{2}$, and $e^{-D}{ }_{i}$ behave in a similar fashion, they only differ in the weight given to the distance differences reflected and quantified by $D_{\mathrm{i}}$.

$$
\begin{aligned}
& S(A \rightarrow B)=\sum_{i=1}^{h} \frac{P}{\left(D_{i}+1\right)^{2}} \\
& S(A \rightarrow B)=\sum_{i=1}^{h} \frac{P}{e^{D_{i}}}
\end{aligned}
$$

Assume that the minimum number of features, $h$, between two comparing windows is found to be 10. The first case in Table 4.4 shows that 5 of these features when superimposed show perfect match (i.e., $\mathrm{D}_{\mathrm{i}}=0, \mathrm{q}=1,2, \ldots, 5$ ), and that the other 5 features show the following pixel distances $\left(\mathrm{D}_{6}=2, \mathrm{D}_{7}=3, \mathrm{D}_{8}=4\right.$, and $\left.\mathrm{D}_{9}=\mathrm{D}_{10}=1\right)$. Three more examples are shown and their corresponding similarity values for Eq. (4.1), (4.3) and (4.4). In Table 4.5, the $D_{\mathrm{i}} \mathrm{s}$ take on other different values so as to get a feel on the different outcomes of these similarity measures. 
Table 4.4 Numerical examples for (4.1), (4.3), and (4.4).

\begin{tabular}{|l|l|l|l|l|l|l|l|l|l|l|l|l|}
\hline $\mathrm{D}_{1}$ & $\mathrm{D}_{2}$ & $\mathrm{D}_{3}$ & $\mathrm{D}_{4}$ & $\mathrm{D}_{5}$ & $\mathrm{D}_{6}$ & $\mathrm{D}_{7}$ & $\mathrm{D}_{8}$ & $\mathrm{D}_{9}$ & $\mathrm{D}_{10}$ & $\mathrm{P} /\left(D_{\mathrm{i}}+1\right)$ & $\mathrm{P} /\left(D_{\mathrm{i}}+1\right)^{2}$ & $\mathrm{P} /\left(\mathrm{e}^{D \mathrm{i}}\right)$ \\
\hline 0 & 0 & 0 & 0 & 0 & 2 & 3 & 4 & 1 & 1 & 0.6783 & 0.5713 & 0.5939 \\
\hline 0 & 0 & 0 & 0 & 0 & 4 & 6 & 8 & 2 & 2 & 0.6120 & 0.5294 & 0.5291 \\
\hline 0 & 0 & 0 & 0 & 0 & 8 & 12 & 16 & 4 & 4 & 0.5646 & 0.5101 & 0.5036 \\
\hline 0 & 0 & 0 & 0 & 0 & 16 & 24 & 32 & 8 & 8 & 0.5351 & 0.5030 & 0.5000 \\
\hline
\end{tabular}

Table 4.5 Numerical examples for (4.1), (4.3), and (4.4).

\begin{tabular}{|l|l|l|l|l|l|l|l|l|l|l|l|l|}
\hline $\mathrm{D}_{1}$ & $\mathrm{D}_{2}$ & $\mathrm{D}_{3}$ & $\mathrm{D}_{4}$ & $\mathrm{D}_{5}$ & $\mathrm{D}_{6}$ & $\mathrm{D}_{7}$ & $\mathrm{D}_{8}$ & $\mathrm{D}_{9}$ & $\mathrm{D}_{10}$ & $\mathrm{P} /\left(D_{\mathrm{i}}+1\right)$ & $\mathrm{P} /\left(D_{\mathrm{i}}+1\right)^{2}$ & $\mathrm{P} / e^{D \mathrm{i}}$ \\
\hline 1 & 1 & 1 & 1 & 1 & 1 & 1 & 1 & 1 & 1 & 0.5 & 0.25 & 0.3678 \\
\hline 2 & 2 & 2 & 2 & 2 & 2 & 2 & 2 & 2 & 2 & 0.3333 & 0.1111 & 0.1353 \\
\hline 4 & 4 & 4 & 4 & 4 & 4 & 4 & 4 & 4 & 4 & 0.2 & 0.04 & 0.0183 \\
\hline 8 & 8 & 8 & 8 & 8 & 8 & 8 & 8 & 8 & 8 & 0.1111 & 0.0123 & 0.0003 \\
\hline 16 & 16 & 16 & 16 & 16 & 16 & 16 & 16 & 16 & 16 & 0.0588 & 0.0035 & $1.125 \mathrm{E}-06$ \\
\hline
\end{tabular}

\subsubsection{Matching Thermal Facial Features}

In section 3.2 we outlined the process to obtain the signatures and templates. We proceeded to apply the similarity measure in the following order:

- $\quad S_{T T}(A \rightarrow B)$, where $A$ and $B$ are thermal facial templates.

- $\quad S_{S 1 T}(A \rightarrow B)$, where $A$ is the first signature set, and $B$ is a template.

- $\quad S_{S 2 T}(A \rightarrow B)$, where $A$ is the second signature set, and $B$ is a template.

- $\quad S_{S 3 T}(A \rightarrow B)$, where $A$ is the third signature set, and $B$ is a template.

- $S_{S 4 T}(A \rightarrow B)$, where $A$ is the fourth signature set, and $B$ is a template.

We remind the reader that each subject has four thermal images taken at different times from which we generated four unique signatures, so we have four signatures for each subject. In section 4.4 we present partial results for the computation of $S_{T T}(A \rightarrow B)$ when $B$ is the template of Subject 10 in the database and $A$ is the template for all subjects in the 
database.

The computation of $S_{T T}, S_{S 1 T}, S_{S 2 T}, S_{S 3 T}$, and $S_{S 4 T}$ was also performed on diffused thermal signatures and templates. The signatures and templates were diffused using the same anisotropic diffusion algorithm as described in chapter 3, the diffusion was done prior to applying the similarity measure in (4.1). The reason for using diffused templates and signatures was to create thicker feature vectors and test the performance of the similarity measure on these new signatures and templates. The results of this experiment yielded higher values of similarity, and the results are provided in chapter 5.

\subsection{Partial Results of Applied Similarity Measure}

This section presents partial results for the computation of $S_{T T}(A \rightarrow B)$ where $B$ is the template of subject 10 shown in figure 4.2, and $A$ is the template for all subjects in the database, shown in figures 4.3-4.15. The images in figures 4.3a-415a show the overlay of $B$ and $A$, the images have been resized to see more clearly the overlay of the templates.

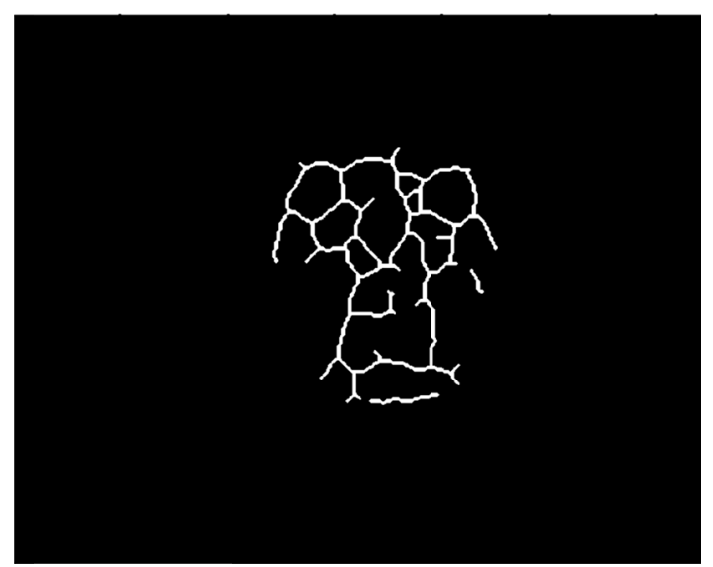

Fig. 4.2 Template of subject 10 


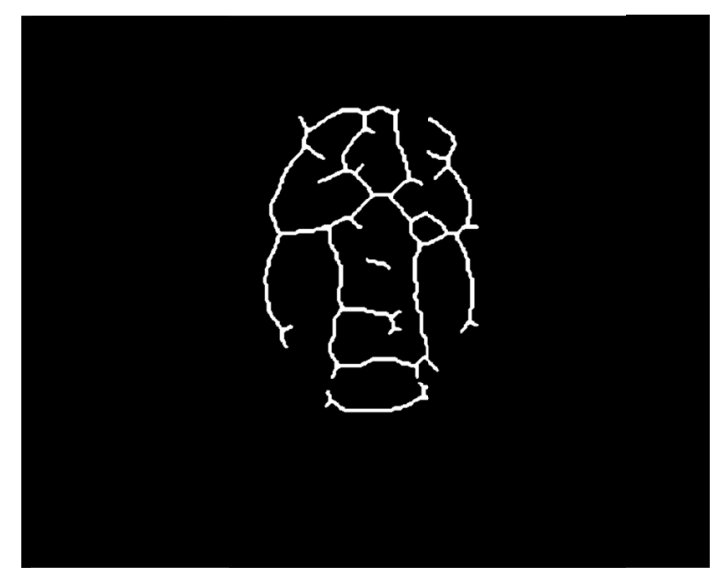

Fig. 4.3 Template of subject 1

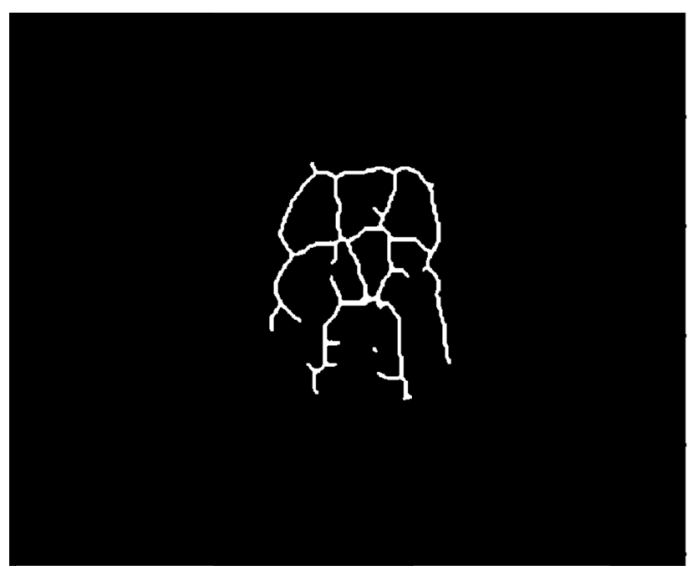

Fig. 4.4 Template of subject 2

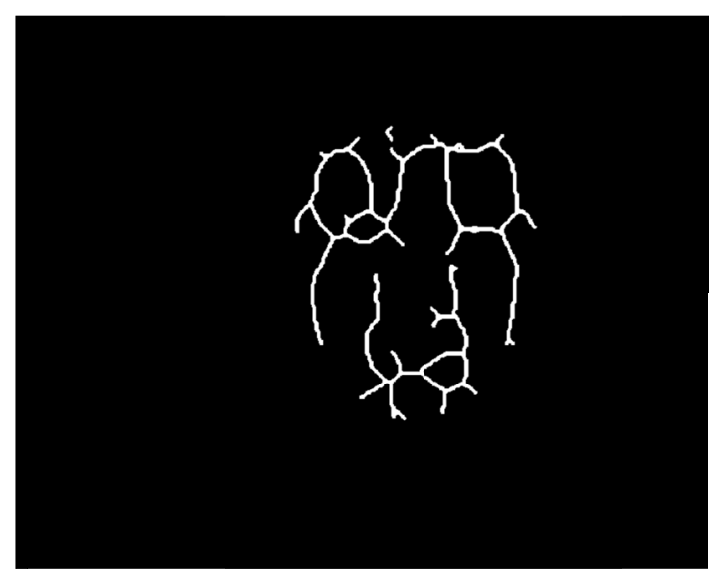

Fig. 4.5 Template of subject 3

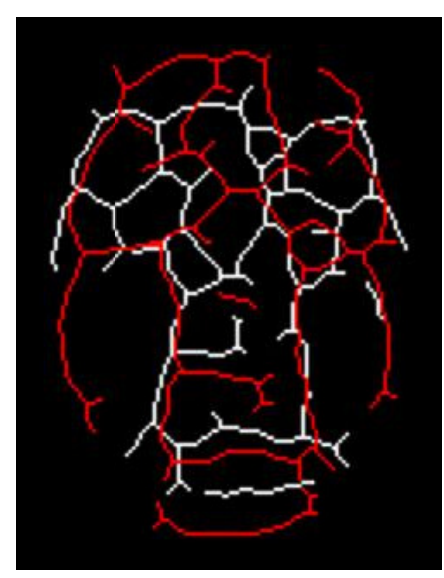

Fig. 4.3a Template of subject 1 (red) overlay with template of subject 10 (white)

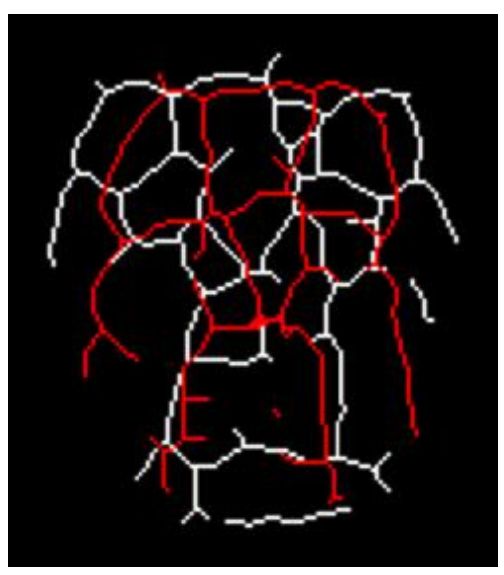

Fig. 4.4a Template of subject 2 (red) overlay with template of subject 10 (white)

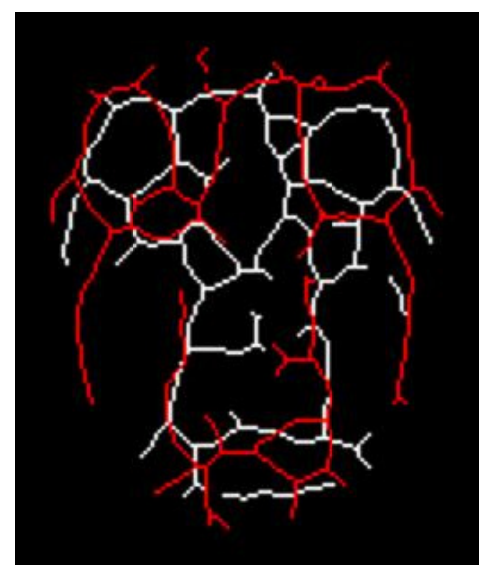

Fig. 4.5a Template of subject 3 (red) overlay with template of subject 10 (white) 


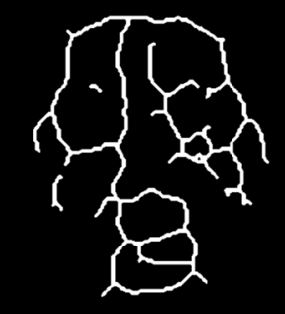

Fig. 4.6 Template of subject 4

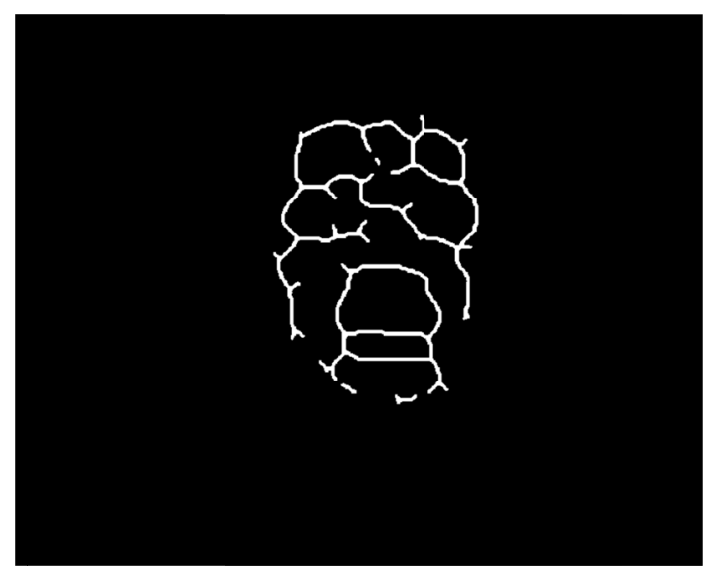

Fig. 4.7 Template of subject 5

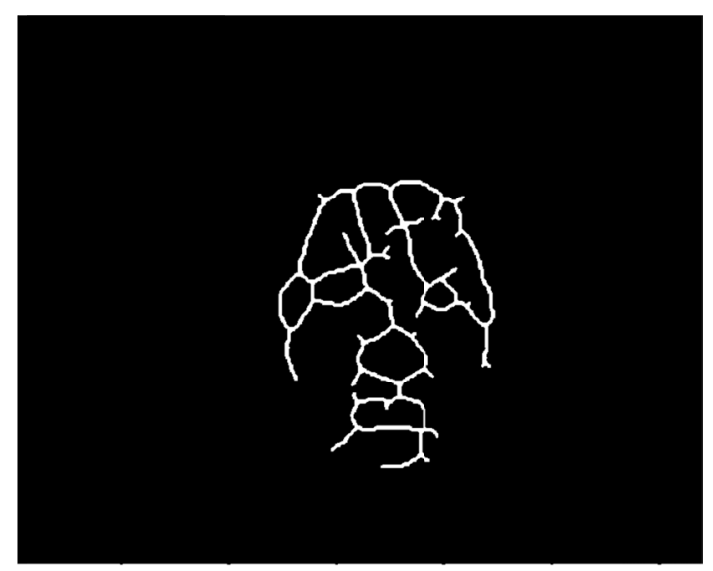

Fig. 4.8 Template of subject 6

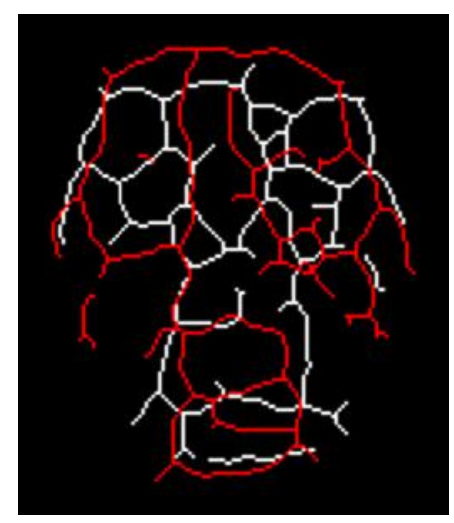

Fig. 4.6a Template of subject 4 (red) overlay with template of subject 10 (white)

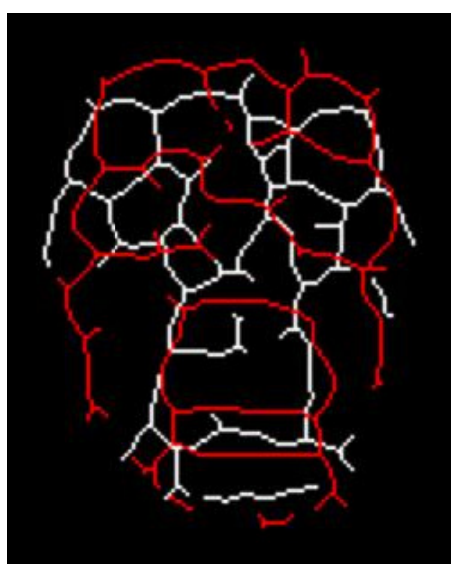

Fig. 4.7a Template of subject 5 (red) overlay with template of subject 10 (white)

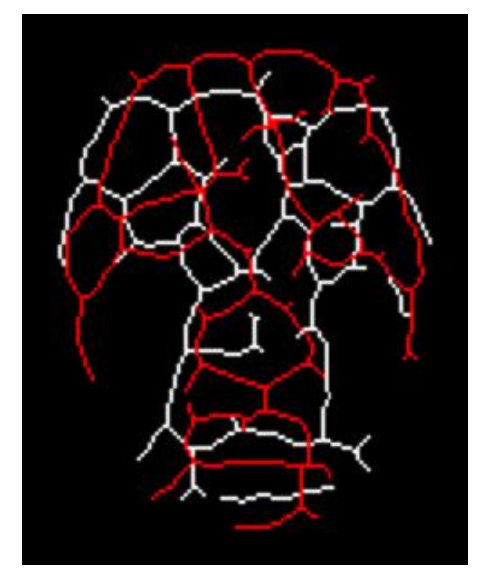

Fig. 4.8a Template of subject 6 (red) overlay with template of subject 10 (white) 


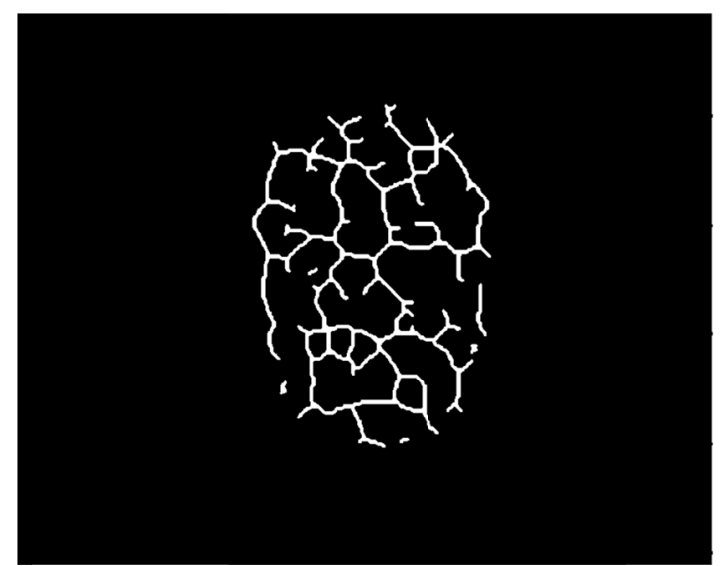

Fig. 4.9 Template of subject 7

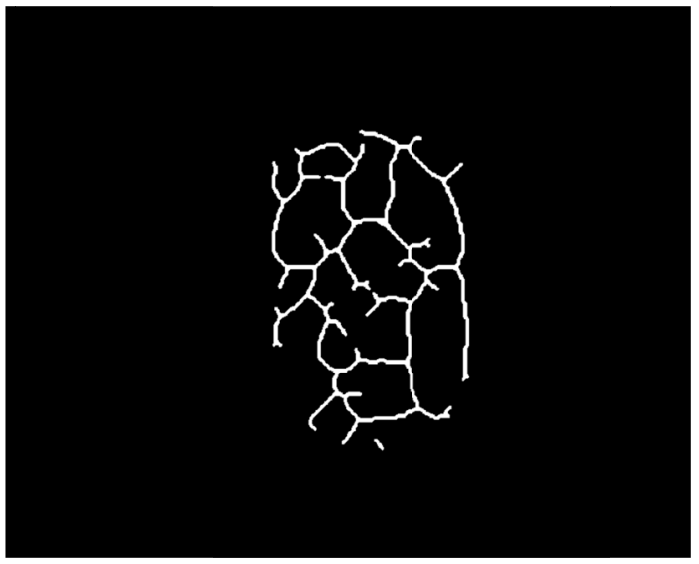

Fig. 4.10 Template of subject 8

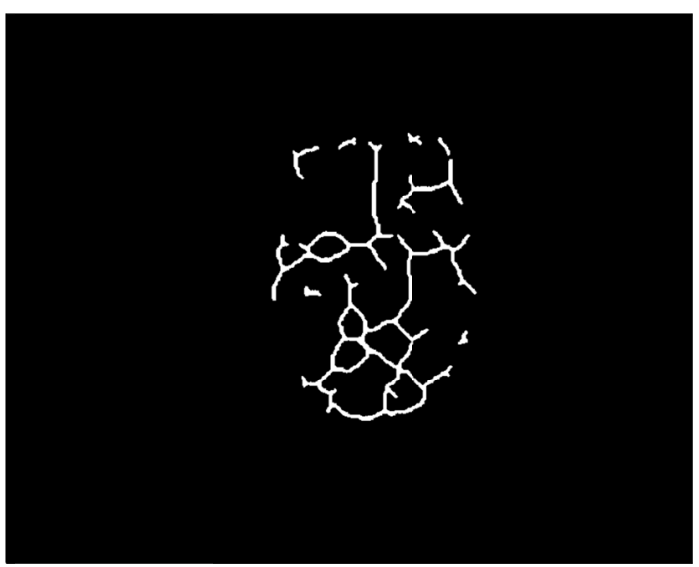

Fig. 4.11 Template of subject 9

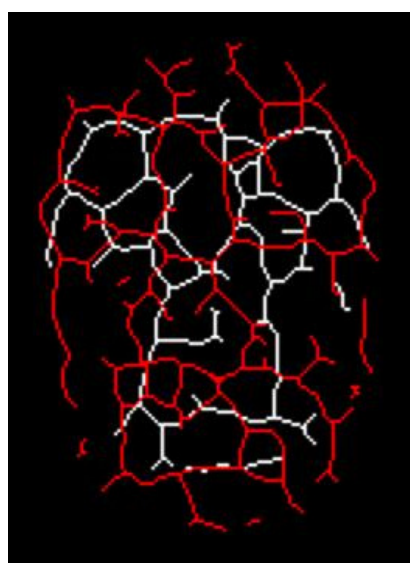

Fig. 4.9a Template of subject 7 (red) overlay with template of subject 10 (white)

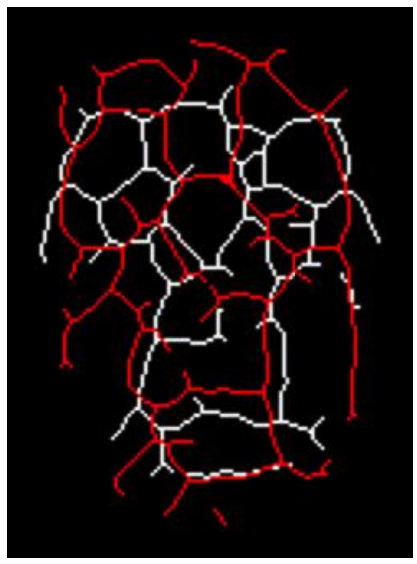

Fig. 4.10a Template of subject 8 (red) overlay with template of subject 10 (white)

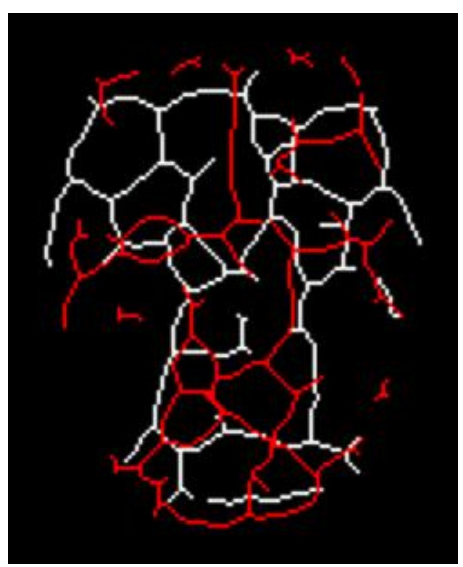

Fig. 4.11a Template of subject 9 (red) overlay with template of subject 10 (white) 


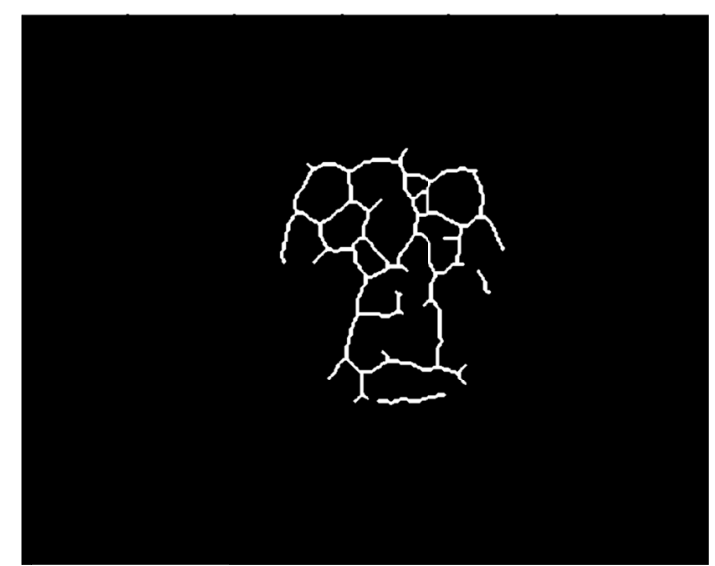

Fig. 4.12 Template of subject 10

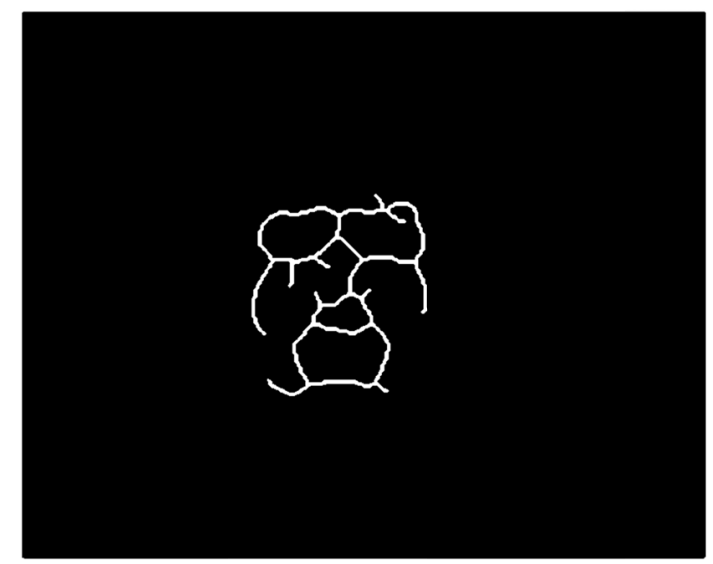

Fig. 4.13 Template of subject 11

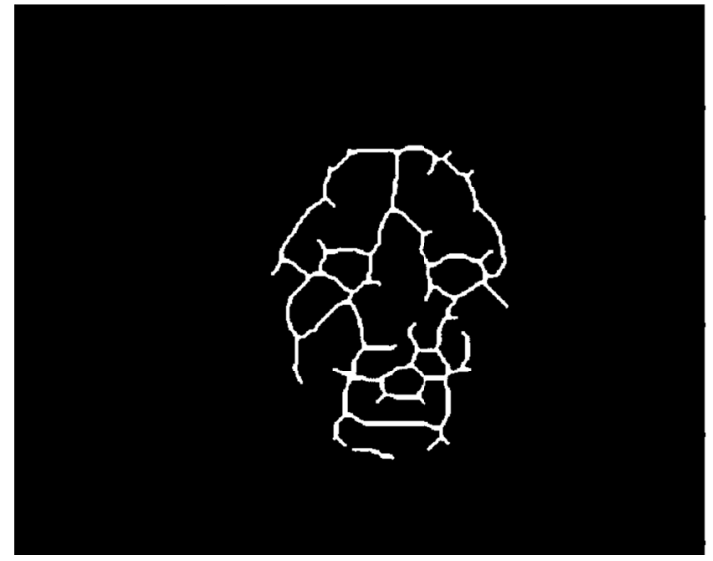

Fig. 4.14 Template of subject 12

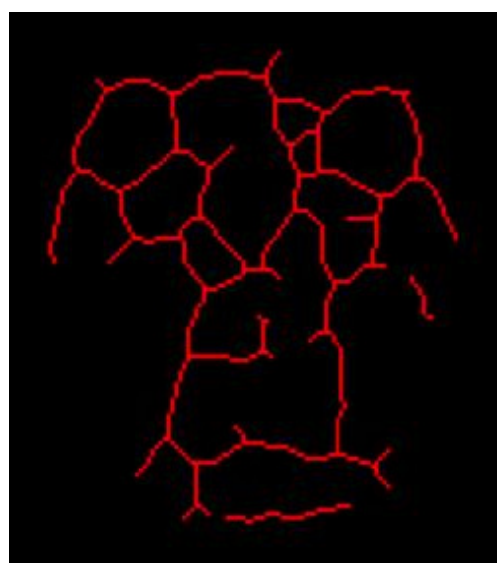

Fig. 4.12a Template of subject 10 (red) overlay with template of subject 10

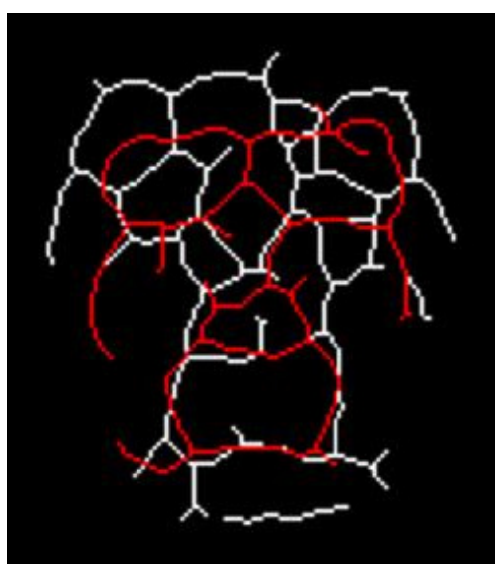

Fig. 4.13a Template of subject 11 (red) overlay with template of subject 10 (white)

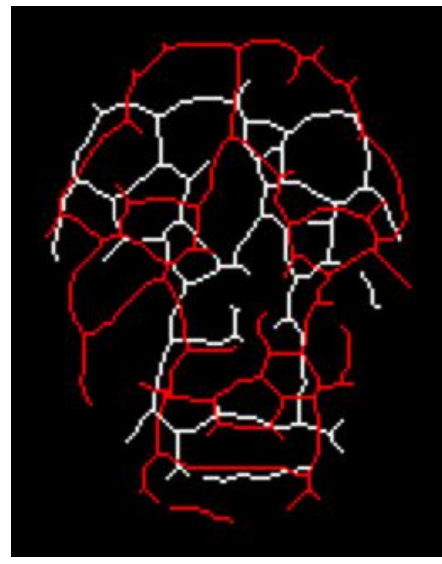

Fig. 4.14a Template of subject 12 (red) overlay with template of subject 10 (white) 


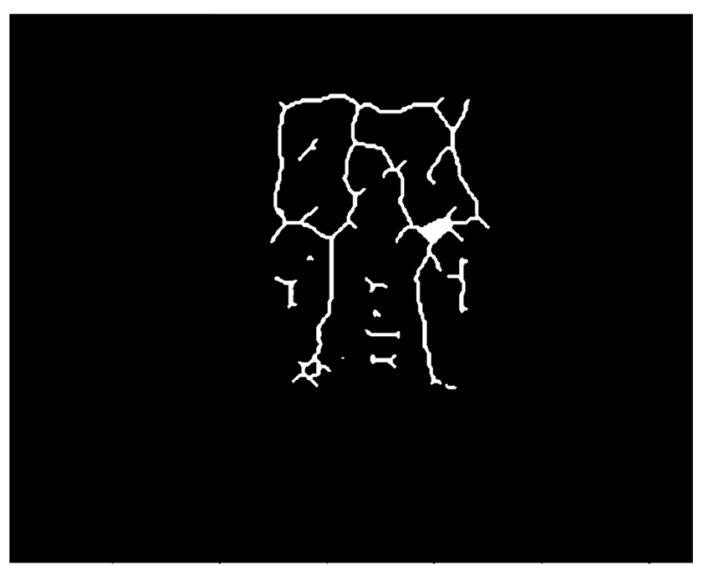

Fig. 4.15 Thermal template of subject 13 .

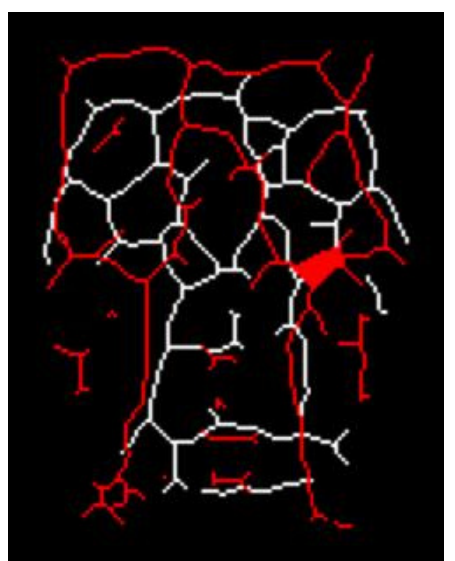

Fig. 4.15a Template of subject 13 (red) overlay with template of subject 10 (white)

In Table 4.4 we show the similarity values obtained from applying the distance-based similarity measure between the reference template of subject 10 and the templates for all subjects in the database. The values show that the template for subject 10 is correctly matched to its own template with a similarity value of 1 or $100 \%$. The next highest value is 0.3414 or $34.14 \%$ similarity between subject 10 and 11 .

Table 4.4 Similarity values for Template vs. Template

\begin{tabular}{|l|c|}
\hline \multicolumn{1}{|c|}{ Template } & $\begin{array}{c}\text { Template Subject 10 as reference. } \\
\text { Similarity Value }\end{array}$ \\
\hline Subject 10 & 1.0000 \\
\hline Subject 11 & 0.3414 \\
\hline Subject 2 & 0.3082 \\
\hline Subject 3 & 0.3057 \\
\hline Subject 9 & 0.2872 \\
\hline Subject 6 & 0.2841 \\
\hline Subject 8 & 0.2825 \\
\hline Subject 4 & 0.2815 \\
\hline Subject 13 & 0.2832 \\
\hline Subject 7 & 0.2649 \\
\hline Subject 12 & 0.2640 \\
\hline Subject 5 & 0.2609 \\
\hline
\end{tabular}

We also obtained the similarity values between templates and signatures from all the 
subjects in the database. In table 4.5 we provide the similarity values for a single subject using the template of subject 10 as the reference image, and thermal facial signatures from all subjects in the database as the non-reference images.

Table 4.5 Similarity values for Signature vs. Template

\begin{tabular}{|l|c|}
\hline \multicolumn{1}{|c|}{ Signature } & $\begin{array}{c}\text { Template Subject 10 as } \\
\text { reference. } \\
\text { Similarity Value }\end{array}$ \\
\hline Subject 10 & 0.4354 \\
\hline Subject 4 & 0.3111 \\
\hline Subject 2 & 0.3053 \\
\hline Subject 5 & 0.2920 \\
\hline Subject 6 & 0.2906 \\
\hline Subject 12 & 0.2876 \\
\hline Subject 10 & 0.2843 \\
\hline Subject 11 & 0.2732 \\
\hline Subject 1 & 0.2706 \\
\hline Subject 3 & 0.2599 \\
\hline Subject 13 & 0.2466 \\
\hline Subject 8 & 0.2417 \\
\hline Subject 7 & 0.2282 \\
\hline
\end{tabular}

The results show that the highest similarity value, 0.4354 or $43.54 \%$, is obtained between the signature and the template of subject 10 , this is a positive match. The similarity value for the positive match between template and signature for subject 10 is less than the positive match between the templates for subject 10 , this is expected as the template vs. template similarity is a one-to-one match and thus the resulting distance between features is zero. However when the template and the signature are compared the one-to-one correspondence no longer exists. In chapter 5 we present and discuss the similarity values obtained for all subjects in the database. 


\section{Chapter 5}

Results and Discussion

\subsection{Introduction}

In chapter 4 the procedure for feature matching using the similarity measure (4.1) on templates and signatures was presented. In this chapter we present and discuss our results. In section 5.2 the similarity values for skeletonized templates and signatures are presented. In section 5.3 we present the similarity results for diffused templates and signatures. In section 5.4 we present intra-subject similarity results.

\subsection{Inter-Subject Results for Skeletonized Templates and Signatures}

\subsubsection{Similarity Results for Matching Template to Templates}

The similarity values for this section are given in tables $5.1-5.5$, the values in the tables are arranged by subject number. In Table 5.1 we present the similarity values for matching template to template for all subjects, we note that when each subject's template is compared to itself the similarity value is 1 , in this case we obtained a positive match for all subjects, thus attaining an accuracy rate of $100 \%$.

Table 5.1 Similarity values for $S_{T T}(A \rightarrow B)$, where $A$ and $B$ are templates. Accuracy $100 \%$

\begin{tabular}{|l|l|l|l|l|l|l|}
\hline & Subj01 & Subj02 & Subj03 & Subj04 & Subj05 & Subj06 \\
\hline T-Subj01 & 1.0000 & 0.3052 & 0.3378 & 0.2899 & 0.3468 & 0.3614 \\
\hline T-Subj02 & 0.2488 & 1.0000 & 0.2248 & 0.2331 & 0.2587 & 0.2538 \\
\hline T-Subj03 & 0.3114 & 0.2355 & 1.0000 & 0.2615 & 0.2834 & 0.2933 \\
\hline T-Subj04 & 0.2840 & 0.2752 & 0.2657 & 1.0000 & 0.3020 & 0.3239 \\
\hline T-Subj05 & 0.3219 & 0.2837 & 0.2857 & 0.2954 & 1.0000 & 0.3236 \\
\hline T-Subj06 & 0.3659 & 0.3149 & 0.3114 & 0.3325 & 0.3423 & 1.0000 \\
\hline T-Subj07 & 0.3117 & 0.3079 & 0.3280 & 0.3194 & 0.3118 & 0.3248 \\
\hline
\end{tabular}




\begin{tabular}{|l|l|l|l|l|l|l|}
\hline & Subj01 & Subj02 & Subj03 & Subj04 & Subj05 & Subj06 \\
\hline T-Subj08 & 0.3686 & 0.3193 & 0.3133 & 0.3300 & 0.3149 & 0.3542 \\
\hline T-Subj09 & 0.2787 & 0.2978 & 0.2673 & 0.3188 & 0.3196 & 0.3232 \\
\hline T-Subj10 & 0.2724 & 0.3082 & 0.3057 & 0.2815 & 0.2609 & 0.2841 \\
\hline T-Subj11 & 0.2045 & 0.3107 & 0.1933 & 0.2226 & 0.2630 & 0.2250 \\
\hline T-Subj12 & 0.3554 & 0.2653 & 0.3310 & 0.2906 & 0.3098 & 0.3096 \\
\hline T-Subj13 & 0.2725 & 0.2800 & 0.2461 & 0.3247 & 0.2541 & 0.2792 \\
\hline
\end{tabular}

\begin{tabular}{|l|l|l|l|l|l|l|l|}
\hline & Subj07 & Subj08 & Subj09 & Subj10 & Subj11 & Subj12 & Subj13 \\
\hline T-Subj01 & 0.2456 & 0.3563 & 0.3041 & 0.3090 & 0.2876 & 0.3469 & 0.2782 \\
\hline T-Subj02 & 0.2037 & 0.2389 & 0.2747 & 0.2780 & 0.3480 & 0.2209 & 0.2165 \\
\hline T-Subj03 & 0.2642 & 0.2764 & 0.2670 & 0.3087 & 0.2421 & 0.2895 & 0.2241 \\
\hline T-Subj04 & 0.2758 & 0.3111 & 0.3220 & 0.2882 & 0.2904 & 0.2682 & 0.3147 \\
\hline T-Subj05 & 0.2294 & 0.2893 & 0.3239 & 0.2665 & 0.3113 & 0.2834 & 0.2361 \\
\hline T-Subj06 & 0.2814 & 0.3440 & 0.3546 & 0.3058 & 0.3122 & 0.3034 & 0.2857 \\
\hline T-Subj07 & 1.0000 & 0.3255 & 0.3213 & 0.3201 & 0.3266 & 0.3249 & 0.3235 \\
\hline T-Subj08 & 0.2972 & 1.0000 & 0.3015 & 0.3061 & 0.2460 & 0.2864 & 0.3228 \\
\hline T-Subj09 & 0.2318 & 0.2592 & 1.0000 & 0.2847 & 0.3513 & 0.2967 & 0.2379 \\
\hline T-Subj10 & 0.2649 & 0.2825 & 0.2872 & 1.0000 & 0.3414 & 0.2640 & 0.2832 \\
\hline T-Subj11 & 0.1962 & 0.1791 & 0.2627 & 0.2806 & 1.0000 & 0.1929 & 0.1709 \\
\hline T-Subj12 & 0.2817 & 0.2849 & 0.3116 & 0.2937 & 0.2602 & 1.0000 & 0.3006 \\
\hline T-Subj13 & 0.2850 & 0.2984 & 0.2800 & 0.2788 & 0.2671 & 0.2915 & 1.0000 \\
\hline
\end{tabular}

In figure 5.1a we show the overlay for the template of subject 1 to its own template and in figure $5.1 \mathrm{~b}$ we show the overlay for the template of subject 1 to the template of subject 11 . Note that the overlay of templates of the same subject are a perfect match thus the overlay shows only one color, when two different templates are overlaid then the reference template is shown in white and the non-reference template is shown in red. A similarity value of 1 or $100 \%$ means that the distances between corresponding features is zero, this is illustrated by figure 5.1a. We note that the number of features is also the same. As it will be shown in the following sections of this chapter the challenge lays on matching the signatures to their corresponding templates as the number and location of features differ 
slightly.

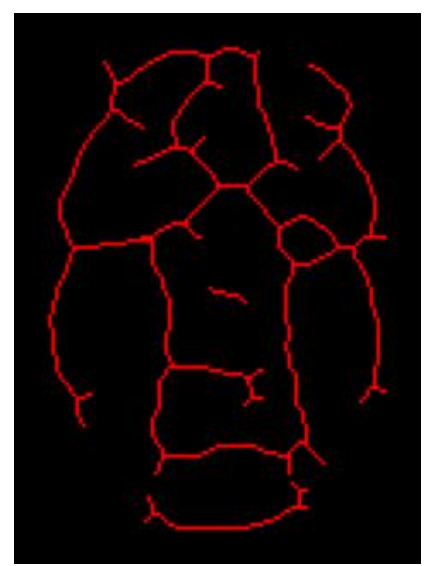

(a)

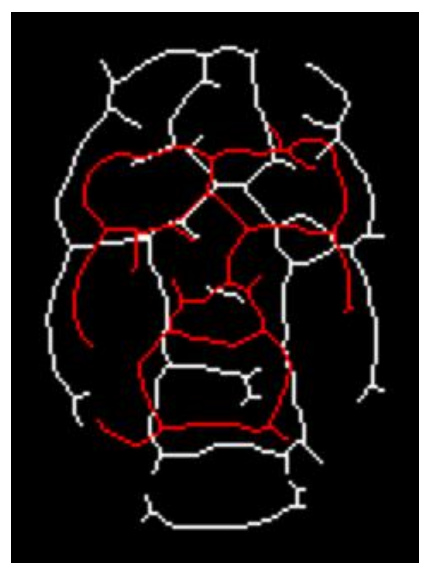

(b)

Fig. 5.1 Overlay of templates of (a) subject 1 to its own template and (b) subject 1 template (white) to the template of subject 11 (red)

Tables 5.2-5.5 present the similarity values for matching signatures to templates, the values in the tables are arranged by subject number, we kept this arrangement to be consistent with Table 5.1 and for simplicity in presenting our results. The diagonal values have been highlighted in yellow, when the value in the diagonal is not the highest for a given subject then the highest value is shown in red and the match is referred to as a negative match. We also provide, for illustrative purposes, images for positive matches and negative matches.

\subsubsection{Similarity Results for Matching the First Signature Set to Templates}

In table 5.2 we have listed the similarity values for $S_{S 1 T}(A \rightarrow B)$, matching the first signature set to the templates. The values in the diagonal for this case are not 1 , the diagonal values range from 0.3097 to 0.6158 , we observe that the diagonal values are the 
highest values for subject 1 through subject 12 thus leading to a positive match between the signature and templates of these subjects. The similarity value for subject 13 is 0.3097 , in the corresponding column we find that the highest value is 0.3108 this means that the first signature of subject 13 was negatively matched to the template of subject 12 . In this case 12 subjects were positively matched, thus obtaining an accuracy rate of $92.31 \%$.

Table 5.2 Similarity values for $S_{S 1 T}(A \rightarrow B)$, where $A$ is the first signature set, and $B$ is a thermal facial template. Accuracy $92.31 \%$

\begin{tabular}{|l|l|l|l|l|l|l|}
\hline & Subj01 & Subj02 & Subj03 & Subj04 & Subj05 & Subj06 \\
\hline T-Subj01 & 0.4957 & 0.3024 & 0.3500 & 0.2999 & 0.3256 & 0.3387 \\
\hline T-Subj02 & 0.2089 & 0.4115 & 0.2138 & 0.2263 & 0.2644 & 0.2244 \\
\hline T-Subj03 & 0.3231 & 0.2823 & 0.4635 & 0.2738 & 0.3016 & 0.2764 \\
\hline T-Subj04 & 0.2642 & 0.2858 & 0.2845 & 0.4916 & 0.3312 & 0.3061 \\
\hline T-Subj05 & 0.2828 & 0.2637 & 0.2610 & 0.2986 & 0.3740 & 0.3343 \\
\hline T-Subj06 & 0.3221 & 0.3174 & 0.3207 & 0.3293 & 0.3510 & 0.5043 \\
\hline T-Subj07 & 0.3199 & 0.2964 & 0.3026 & 0.2990 & 0.3095 & 0.3281 \\
\hline T-Subj08 & 0.3149 & 0.2921 & 0.3000 & 0.3334 & 0.3180 & 0.3350 \\
\hline T-Subj09 & 0.2706 & 0.3053 & 0.2599 & 0.3111 & 0.2920 & 0.2906 \\
\hline T-Subj10 & 0.2773 & 0.3184 & 0.2974 & 0.2715 & 0.2824 & 0.2746 \\
\hline T-Subj11 & 0.2184 & 0.2990 & 0.2040 & 0.2039 & 0.2464 & 0.2311 \\
\hline T-Subj12 & 0.3536 & 0.2851 & 0.2845 & 0.3122 & 0.3072 & 0.3218 \\
\hline T-Subj13 & 0.2696 & 0.2952 & 0.2833 & 0.2888 & 0.2936 & 0.2547 \\
\hline
\end{tabular}

\begin{tabular}{|l|l|l|l|l|l|l|l|}
\hline & Subj07 & Subj08 & Subj09 & Subj10 & Subj11 & Subj12 & Subj13 \\
\hline T-Subj01 & 0.2261 & 0.2852 & 0.2993 & 0.2940 & 0.2528 & 0.3420 & 0.2951 \\
\hline T-Subj02 & 0.1779 & 0.2138 & 0.2144 & 0.2718 & 0.3181 & 0.1975 & 0.2087 \\
\hline T-Subj03 & 0.2617 & 0.2489 & 0.2834 & 0.3075 & 0.2600 & 0.2859 & 0.2550 \\
\hline T-Subj04 & 0.2483 & 0.2880 & 0.3246 & 0.2917 & 0.2728 & 0.2806 & 0.2620 \\
\hline T-Subj05 & 0.2065 & 0.2817 & 0.3240 & 0.2682 & 0.2898 & 0.2880 & 0.2682 \\
\hline T-Subj06 & 0.2422 & 0.3022 & 0.3391 & 0.2956 & 0.3174 & 0.3306 & 0.3029 \\
\hline T-Subj07 & 0.4178 & 0.3349 & 0.3228 & 0.3258 & 0.3154 & 0.3110 & 0.2947 \\
\hline T-Subj08 & 0.2769 & 0.4648 & 0.2822 & 0.2776 & 0.2893 & 0.3050 & 0.3096 \\
\hline T-Subj09 & 0.2282 & 0.2417 & 0.4354 & 0.2843 & 0.2732 & 0.2876 & 0.2466 \\
\hline T-Subj10 & 0.2449 & 0.2505 & 0.2797 & 0.4416 & 0.2851 & 0.2597 & 0.2699 \\
\hline
\end{tabular}




\begin{tabular}{|l|l|l|l|l|l|l|l|}
\hline & Subj07 & Subj08 & Subj09 & Subj10 & Subj11 & Subj12 & Subj13 \\
\hline T-Subj11 & 0.1668 & 0.1730 & 0.2651 & 0.2659 & 0.3376 & 0.1983 & 0.1959 \\
\hline T-Subj12 & 0.2470 & 0.3249 & 0.3169 & 0.2993 & 0.2702 & 0.6158 & 0.3108 \\
\hline T-Subj13 & 0.2703 & 0.3050 & 0.2868 & 0.2923 & 0.2648 & 0.2698 & 0.3097 \\
\hline
\end{tabular}

In figure 5.2 we show the overlay of the template and signature corresponding to a positive match for subjects 12,6 and 1, these subjects had the highest similarity values in table 5.2, $[0.6158,0.5043,0.4957]$ respectively. The templates are shown in white and the signatures are shown in red.

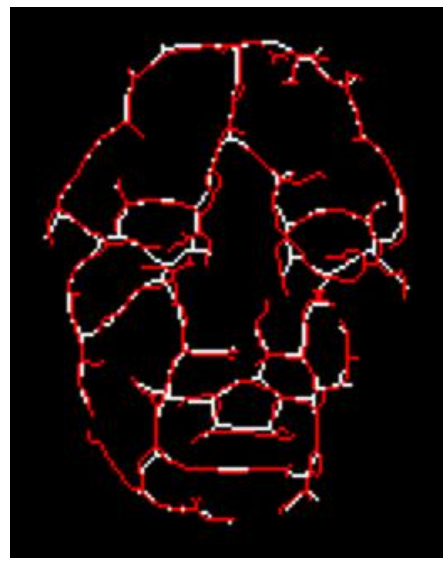

(a)

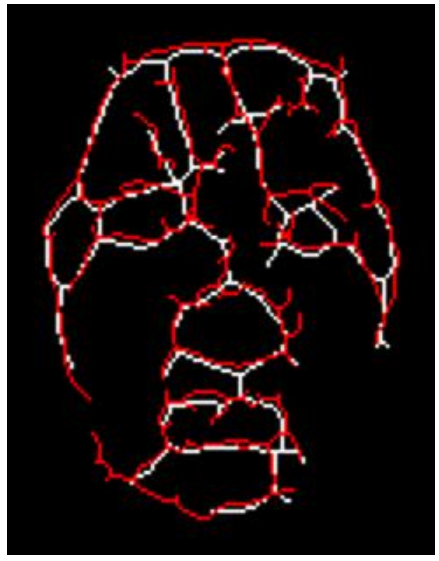

(b)

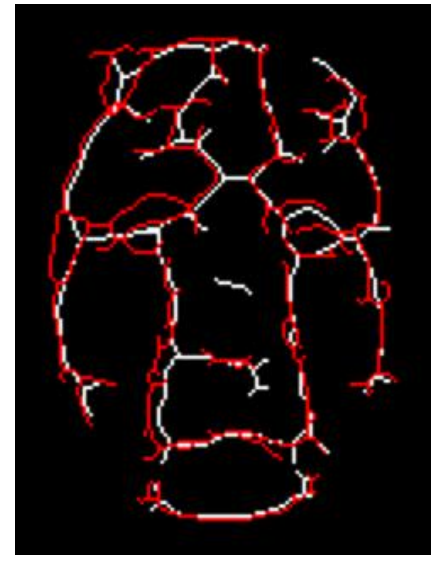

(c)

Fig. 5.2 Overlay of template (white) and signature (red) for positive match of (a) subject 12 , (b) subject 6 , and (c) subject 1 , with similarity values of [0.6158, 0.5043, 0.4957], respectively

In figure 5.3 we present the overlay of the template and signature for the negative match of subject 13. In this case figure 5.3a shows the overlay of the template and signature of subject 13 , similarity value is 0.3097 . Figure $5.3 \mathrm{~b}$ shows the overlay of the template for subject 12 to which the signature of subject 13 was negatively matched with a similarity value of 0.3108 . The templates are shown in white and the signatures are shown in red. 


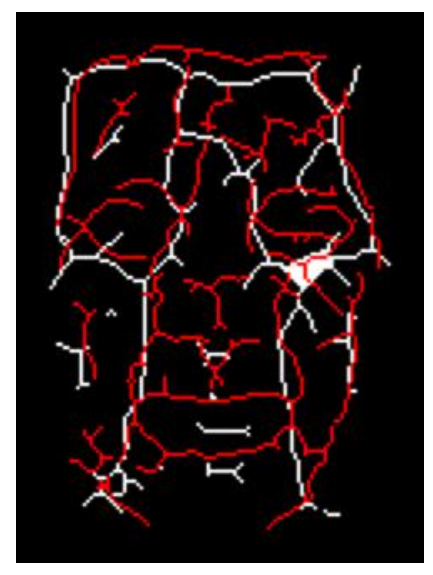

(a)

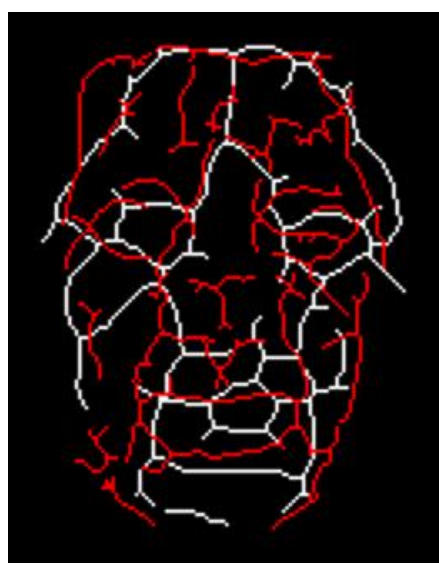

(b)

Fig. 5.3 (a) Subject 13's overlay of its own template (white) and signature (red), similarity value 0.3097 , (b) negative match of the signature of subject 13 to the template of subject 12 with a similarity value of 0.3108

\subsubsection{Similarity Results for Matching the Second Signature Set to Templates}

In table 5.3 we have listed the similarity values for $S_{S 2 T}(A \rightarrow B)$, matching the second set of signatures to the templates. The range of the similarity values in the diagonal is 0.2972-0.5806, we observe that these values are the highest for ten subjects: $1,2,4-8$, 10-12. Subjects 3, 9 and 13 were negatively matched to the templates of subjects 12,2 and 7 respectively. We obtained an accuracy rate of $76.92 \%$ for this case.

Table 5.3 Similarity values for $S_{S 2 T}(A \rightarrow B), A$ is the second signature set, and $B$ is a template. Accuracy $76.92 \%$

\begin{tabular}{|l|l|l|l|l|l|l|}
\hline & Subj01 & Subj02 & Subj03 & Subj04 & Subj05 & Subj06 \\
\hline T-Subj01 & 0.4064 & 0.2640 & 0.3304 & 0.3043 & 0.3263 & 0.3269 \\
\hline T-Subj02 & 0.1997 & 0.3644 & 0.2248 & 0.2457 & 0.2358 & 0.2292 \\
\hline T-Subj03 & 0.3141 & 0.2370 & 0.3001 & 0.2593 & 0.2859 & 0.2883 \\
\hline T-Subj04 & 0.2602 & 0.2933 & 0.2920 & 0.4161 & 0.3154 & 0.3191 \\
\hline T-Subj05 & 0.2766 & 0.2871 & 0.2768 & 0.2909 & 0.3784 & 0.3396 \\
\hline T-Subj06 & 0.3037 & 0.3136 & 0.3135 & 0.3410 & 0.3509 & 0.5806 \\
\hline T-Subj07 & 0.3391 & 0.2843 & 0.3168 & 0.3050 & 0.3103 & 0.3377 \\
\hline T-Subj08 & 0.3158 & 0.2769 & 0.3066 & 0.3506 & 0.3028 & 0.3340 \\
\hline T-Subj09 & 0.2505 & 0.2862 & 0.2792 & 0.2741 & 0.3074 & 0.3135 \\
\hline
\end{tabular}




\begin{tabular}{|l|l|l|l|l|l|l|}
\hline & Subj01 & Subj02 & Subj03 & Subj04 & Subj05 & Subj06 \\
\hline T-Subj10 & 0.2861 & 0.3100 & 0.2729 & 0.2775 & 0.2778 & 0.2658 \\
\hline T-Subj11 & 0.2094 & 0.2908 & 0.2154 & 0.1894 & 0.2446 & 0.2341 \\
\hline T-Subj12 & 0.3457 & 0.2777 & $\mathbf{0 . 3 5 4 1}$ & 0.3128 & 0.3027 & 0.3318 \\
\hline T-Subj13 & 0.2964 & 0.2661 & 0.2572 & 0.2952 & 0.2721 & 0.2541 \\
\hline
\end{tabular}

\begin{tabular}{|l|l|l|l|l|l|l|l|}
\hline & Subj07 & Subj08 & Subj09 & Subj10 & Subj11 & Subj12 & Subj13 \\
\hline T-Subj01 & 0.2321 & 0.2771 & 0.2564 & 0.2885 & 0.2715 & 0.3506 & 0.2837 \\
\hline T-Subj02 & 0.1931 & 0.2102 & 0.3233 & 0.2509 & 0.3172 & 0.2067 & 0.2521 \\
\hline T-Subj03 & 0.2631 & 0.2655 & 0.2321 & 0.3093 & 0.2767 & 0.2753 & 0.2864 \\
\hline T-Subj04 & 0.2462 & 0.2864 & 0.2747 & 0.2799 & 0.2550 & 0.2792 & 0.2763 \\
\hline T-Subj05 & 0.2077 & 0.2803 & 0.2611 & 0.2608 & 0.2965 & 0.2878 & 0.2970 \\
\hline T-Subj06 & 0.2562 & 0.3031 & 0.3114 & 0.3075 & 0.3111 & 0.3253 & 0.2592 \\
\hline T-Subj07 & 0.4345 & 0.3387 & 0.3118 & 0.3295 & 0.3129 & 0.3042 & 0.3356 \\
\hline T-Subj08 & 0.2832 & 0.4351 & 0.2575 & 0.2883 & 0.2785 & 0.2987 & 0.3252 \\
\hline T-Subj09 & 0.2270 & 0.2450 & 0.2972 & 0.2870 & 0.2847 & 0.2805 & 0.2775 \\
\hline T-Subj10 & 0.2600 & 0.2454 & 0.3069 & 0.4389 & 0.3076 & 0.2655 & 0.2766 \\
\hline T-Subj11 & 0.1727 & 0.1794 & 0.2564 & 0.2626 & 0.3314 & 0.2106 & 0.2115 \\
\hline T-Subj12 & 0.2629 & 0.3321 & 0.2774 & 0.3129 & 0.2728 & 0.5661 & 0.2785 \\
\hline T-Subj13 & 0.2806 & 0.3076 & 0.2755 & 0.2727 & 0.2812 & 0.2679 & 0.3311 \\
\hline
\end{tabular}

In figure 5.4 we show the overlay of the template and signature corresponding to a positive match for subjects 12,6 and 8 , these subjects had the highest similarity values in table $5.3,[0.56610 .5806,0.4351]$ respectively. The templates are shown in white and the signatures are shown in red. 


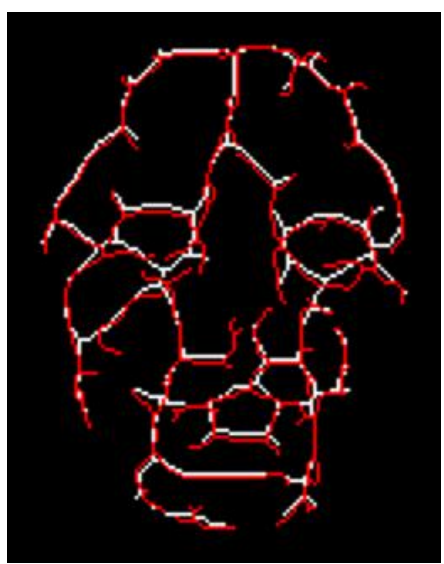

(a)

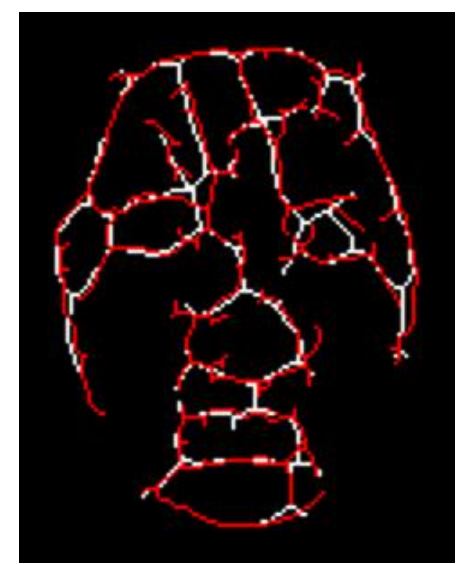

(b)

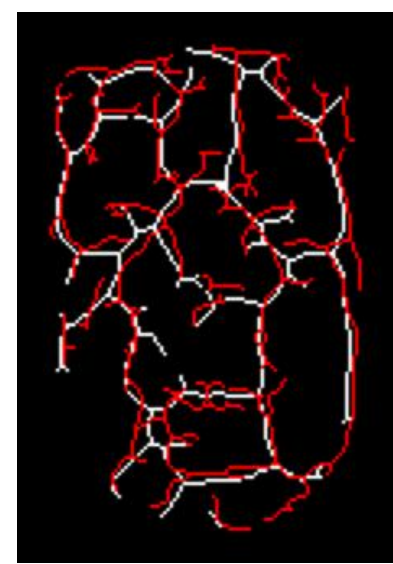

(c)

Fig. 5.4 Overlay of template (white) and signature (red) for positive match of (a) subject 12 , (b) subject 6 , and (c) subject 8 , with similarity values of [0.5661 $0.5806,0.4351]$ respectively

In figure 5.5 we present the overlay of the template and signature for the negative match of subject 3 . In this case figure 5.5a shows the overlay of the template and signature of subject 3 , similarity value is 0.3001 . Figure $5.5 \mathrm{~b}$ shows the overlay of the template for subject 12 to which the signature of subject 3 was negatively matched with a similarity value of 0.3541 . The templates are shown in white and the signatures are shown in red.

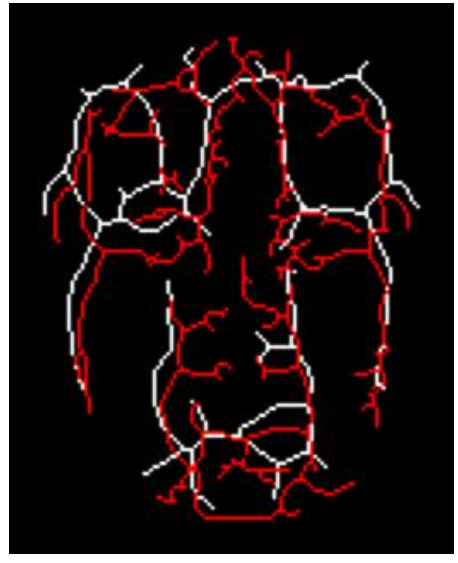

(a)

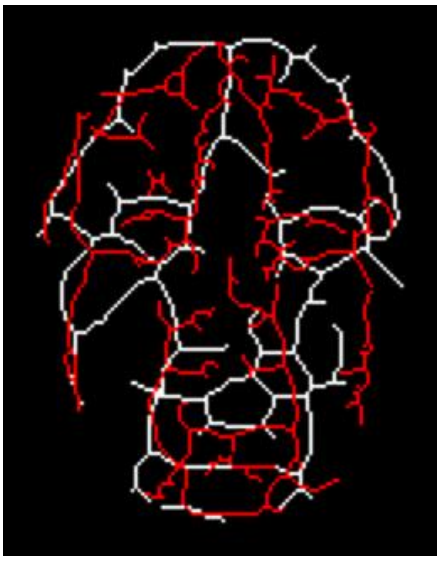

(b)

Fig. 5.5 (a) Subject 3's overlay of its own template and signature, similarity value 0.3001 .

(b) negative match of the signature of subject 3 to the template of subject 12 with a similarity value of 0.3541 . 


\subsubsection{Similarity Results for Matching the Third Signature Set to Templates}

In table 5.4 we have listed the similarity values for $S_{S 3 T}(A \rightarrow B)$, matching the third set of signatures to the templates. The diagonal values in this table ranges from 0.2936 to 0.5132 , in this case we notice that there is only one negative match, the third signature of subject 9 is matched to the template of subject 7 . The signatures of the other twelve subjects are positively matched to their corresponding template, thus obtaining an accuracy rate of $92.31 \%$.

In figure 5.6 we show the overlay of the template and signature corresponding to a positive match for subjects 12,4 and 6 , these subjects had the highest similarity values in table $5.4,[0.5132,0.4606,0.4556]$ respectively. The templates are shown in white and the signatures are shown in red.

Table 5.4 Similarity values for $S_{S 3 T}(A \rightarrow B)$, where $A$ is the third signature set, and $B$ is a thermal facial template. Accuracy $92.31 \%$

\begin{tabular}{|l|l|l|l|l|l|l|}
\hline & Subj01 & Subj02 & Subj03 & Subj04 & Subj05 & Subj06 \\
\hline T-Subj01 & 0.4190 & 0.2724 & 0.3080 & 0.2822 & 0.3119 & 0.2942 \\
\hline T-Subj02 & 0.2464 & 0.3898 & 0.2068 & 0.2147 & 0.2785 & 0.2978 \\
\hline T-Subj03 & 0.2583 & 0.2427 & 0.3883 & 0.2765 & 0.2702 & 0.2647 \\
\hline T-Subj04 & 0.2962 & 0.2828 & 0.2877 & 0.4606 & 0.2924 & 0.3159 \\
\hline T-Subj05 & 0.3465 & 0.2583 & 0.2619 & 0.2746 & 0.4502 & 0.3015 \\
\hline T-Subj06 & 0.3702 & 0.3110 & 0.2974 & 0.3080 & 0.3507 & 0.4556 \\
\hline T-Subj07 & 0.3168 & 0.2783 & 0.3324 & 0.3127 & 0.3218 & 0.3329 \\
\hline T-Subj08 & 0.3338 & 0.2974 & 0.2820 & 0.3211 & 0.2961 & 0.3406 \\
\hline T-Subj09 & 0.2917 & 0.2802 & 0.2749 & 0.3144 & 0.2961 & 0.3162 \\
\hline T-Subj10 & 0.2175 & 0.3095 & 0.2770 & 0.2864 & 0.2622 & 0.2710 \\
\hline T-Subj11 & 0.2232 & 0.2657 & 0.1830 & 0.2124 & 0.2736 & 0.2433 \\
\hline T-Subj12 & 0.3430 & 0.2729 & 0.3487 & 0.2876 & 0.3016 & 0.3046 \\
\hline T-Subj13 & 0.2457 & 0.2707 & 0.2452 & 0.3071 & 0.2613 & 0.3040 \\
\hline
\end{tabular}




\begin{tabular}{|l|l|l|l|l|l|l|l|}
\hline & Subj07 & Subj08 & Subj09 & Subj10 & Subj11 & Subj12 & Subj13 \\
\hline T-Subj01 & 0.2651 & 0.2741 & 0.2465 & 0.2549 & 0.2722 & 0.3389 & 0.2331 \\
\hline T-Subj02 & 0.2088 & 0.2095 & 0.1860 & 0.2693 & 0.2949 & 0.2292 & 0.1707 \\
\hline T-Subj03 & 0.2910 & 0.2643 & 0.2234 & 0.2855 & 0.2625 & 0.2811 & 0.2257 \\
\hline T-Subj04 & 0.2752 & 0.2715 & 0.2535 & 0.2692 & 0.2624 & 0.2827 & 0.2843 \\
\hline T-Subj05 & 0.2471 & 0.2545 & 0.2322 & 0.2612 & 0.2988 & 0.2986 & 0.2167 \\
\hline T-Subj06 & 0.2579 & 0.2773 & 0.2560 & 0.2968 & 0.2998 & 0.3337 & 0.2602 \\
\hline T-Subj07 & 0.3836 & 0.3163 & 0.3346 & 0.3077 & 0.3039 & 0.3168 & 0.3347 \\
\hline T-Subj08 & 0.2917 & 0.3771 & 0.2871 & 0.3079 & 0.2611 & 0.2956 & 0.2884 \\
\hline T-Subj09 & 0.2408 & 0.2528 & 0.2936 & 0.2442 & 0.3075 & 0.2892 & 0.2454 \\
\hline T-Subj10 & 0.2640 & 0.2674 & 0.2158 & 0.3565 & 0.3110 & 0.2720 & 0.2553 \\
\hline T-Subj11 & 0.1930 & 0.1873 & 0.1632 & 0.2771 & 0.3810 & 0.2138 & 0.1703 \\
\hline T-Subj12 & 0.2894 & 0.3287 & 0.2980 & 0.3150 & 0.2701 & 0.5132 & 0.2982 \\
\hline T-Subj13 & 0.2901 & 0.2623 & 0.2435 & 0.2557 & 0.2701 & 0.2803 & 0.3711 \\
\hline
\end{tabular}

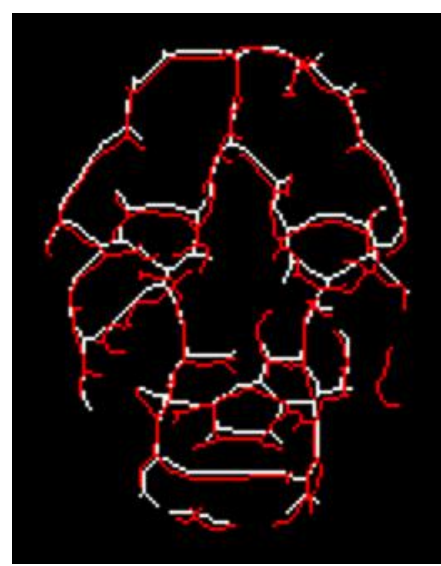

(a)

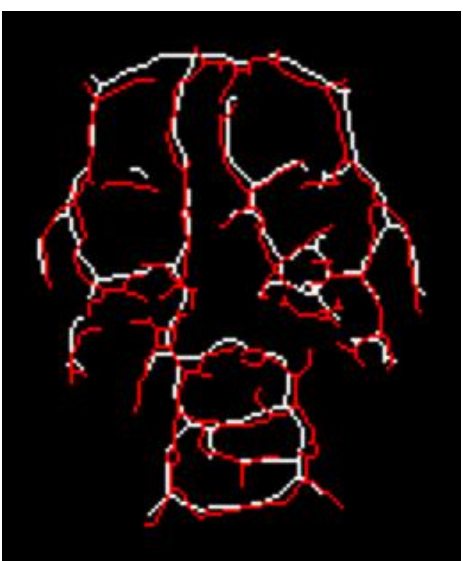

(b)

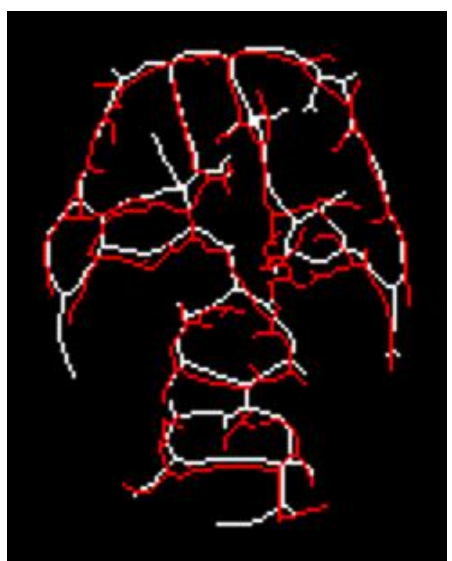

(c)

Fig. 5.6 Overlay of template (white) and signature (red) for positive match of (a) subject

12 , (b) subject 4 , and (c) subject 6 , with similarity values of [0.5132, $0.4606,0.4556]$ respectively

In figure 5.7 we present the overlay of the template and signature for the negative match of subject 9. In this case figure 5.7 a shows the overlay of the template and signature of subject 9 , similarity value is 0.2936 . Figure $5.7 \mathrm{~b}$ shows the overlay for the template of subject 7 to which the signature of subject 9 was negatively matched with a similarity value of 0.3346 . The templates are shown in white and the signatures are shown in red. 


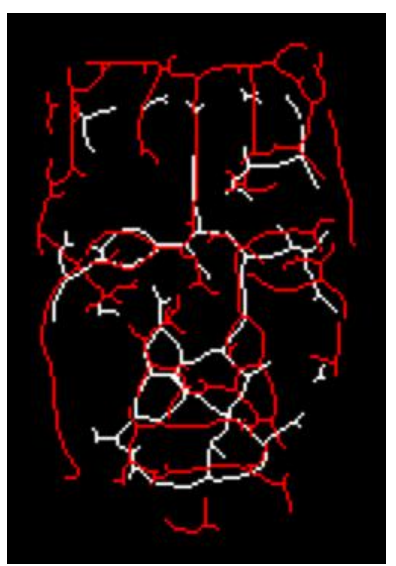

(a)

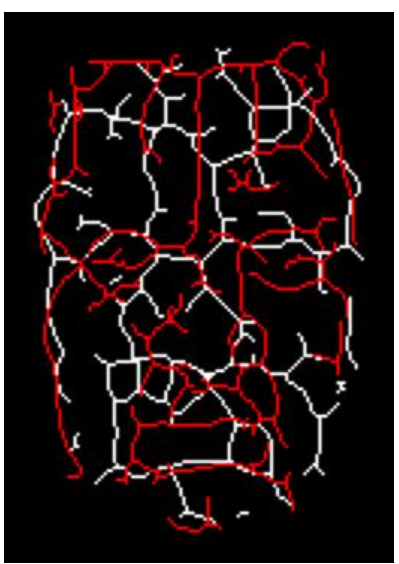

(b)

Fig. 5.7 (a) Subject 9's overlay of its own template and signature, similarity value 0.2936,

(b) negative match of the signature of subject 9 to the template of subject 7 with a similarity value of 0.3346

\subsubsection{Similarity Results for Matching the Fourth Signature Set to Templates}

In table 5.5 we have listed the similarity values for $S_{S 4 T}(A \rightarrow B)$, matching the fourth set of signatures to the templates. The diagonal values in this table range from 0.3179 to 0.5252. in this case we notice that there is only one negative match, the third signature of subject 4 was matched to subject 12, and subjects 1-3, 5-12 were positively matched.

In figure 5.8 we show the overlay of the template and signature corresponding to a positive match for subjects 6,3 , and 12; these subjects had the highest similarity values in table $5.5,[0.5252,0.4986,0.4955]$ respectively. The templates are shown in white and the signatures are shown in red. 
Table 5.5 Similarity values for $S_{S 4 T}(A \rightarrow B)$, where $A$ is the fourth signature set, and $B$ is a template. Accuracy $92.31 \%$.

\begin{tabular}{|l|l|l|l|l|l|l|}
\hline & Subj01 & Subj02 & Subj03 & Subj04 & Subj05 & Subj06 \\
\hline T-Subj01 & 0.4280 & 0.2845 & 0.3364 & 0.2871 & 0.3195 & 0.3061 \\
\hline T-Subj02 & 0.2234 & 0.4113 & 0.2059 & 0.2284 & 0.2698 & 0.2492 \\
\hline T-Subj03 & 0.2555 & 0.2648 & 0.4986 & 0.2651 & 0.2619 & 0.2693 \\
\hline T-Subj04 & 0.3002 & 0.2800 & 0.2954 & 0.3179 & 0.2923 & 0.3247 \\
\hline T-Subj05 & 0.3443 & 0.2824 & 0.2821 & 0.3231 & 0.4437 & 0.2977 \\
\hline T-Subj06 & 0.3777 & 0.3364 & 0.3114 & 0.3319 & 0.3621 & 0.5252 \\
\hline T-Subj07 & 0.3246 & 0.2887 & 0.3090 & 0.3033 & 0.3021 & 0.3339 \\
\hline T-Subj08 & 0.3360 & 0.2933 & 0.2844 & 0.2899 & 0.2862 & 0.3375 \\
\hline T-Subj09 & 0.2913 & 0.3075 & 0.2615 & 0.3059 & 0.2997 & 0.3060 \\
\hline T-Subj10 & 0.2318 & 0.3132 & 0.3120 & 0.3089 & 0.2502 & 0.2551 \\
\hline T-Subj11 & 0.2157 & 0.2983 & 0.1925 & 0.2387 & 0.2616 & 0.2346 \\
\hline T-Subj12 & 0.3344 & 0.2654 & 0.3170 & 0.3348 & 0.3011 & 0.3092 \\
\hline T-Subj13 & 0.2491 & 0.2847 & 0.2469 & 0.2889 & 0.2401 & 0.2579 \\
\hline
\end{tabular}

\begin{tabular}{|l|l|l|l|l|l|l|l|}
\hline & Subj07 & Subj08 & Subj09 & Subj10 & Subj11 & Subj12 & Subj13 \\
\hline T-Subj01 & 0.2660 & 0.2925 & 0.3144 & 0.2737 & 0.2747 & 0.3222 & 0.2321 \\
\hline T-Subj02 & 0.2043 & 0.2268 & 0.2805 & 0.2506 & 0.3192 & 0.2038 & 0.1694 \\
\hline T-Subj03 & 0.2809 & 0.2536 & 0.2701 & 0.2892 & 0.2468 & 0.2668 & 0.2151 \\
\hline T-Subj04 & 0.2867 & 0.3037 & 0.2991 & 0.3095 & 0.3003 & 0.2605 & 0.2962 \\
\hline T-Subj05 & 0.2481 & 0.3061 & 0.3245 & 0.2486 & 0.2983 & 0.2804 & 0.2101 \\
\hline T-Subj06 & 0.2658 & 0.3070 & 0.3142 & 0.3135 & 0.3406 & 0.2943 & 0.2748 \\
\hline T-Subj07 & 0.3828 & 0.3180 & 0.3251 & 0.3181 & 0.3484 & 0.3162 & 0.3454 \\
\hline T-Subj08 & 0.2871 & 0.3695 & 0.2867 & 0.3013 & 0.2729 & 0.3023 & 0.2895 \\
\hline T-Subj09 & 0.2275 & 0.2648 & 0.4330 & 0.2723 & 0.3133 & 0.2773 & 0.2375 \\
\hline T-Subj10 & 0.2308 & 0.2392 & 0.2891 & 0.3849 & 0.3330 & 0.2703 & 0.2435 \\
\hline T-Subj11 & 0.1631 & 0.2008 & 0.2723 & 0.2553 & 0.4568 & 0.1963 & 0.1652 \\
\hline T-Subj12 & 0.2798 & 0.2965 & 0.3229 & 0.3030 & 0.2939 & 0.4955 & 0.2897 \\
\hline T-Subj13 & 0.2790 & 0.2526 & 0.2927 & 0.3046 & 0.2726 & 0.2566 & 0.3757 \\
\hline
\end{tabular}




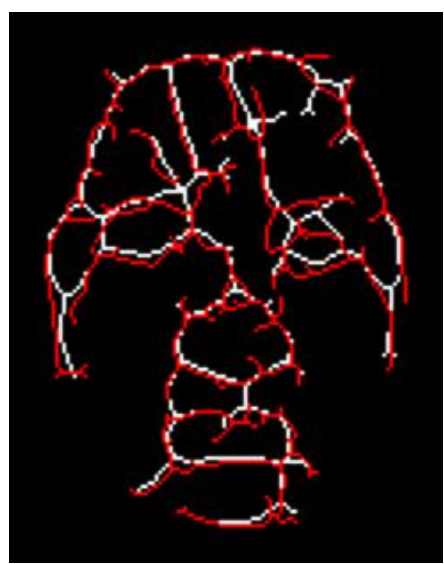

(a)

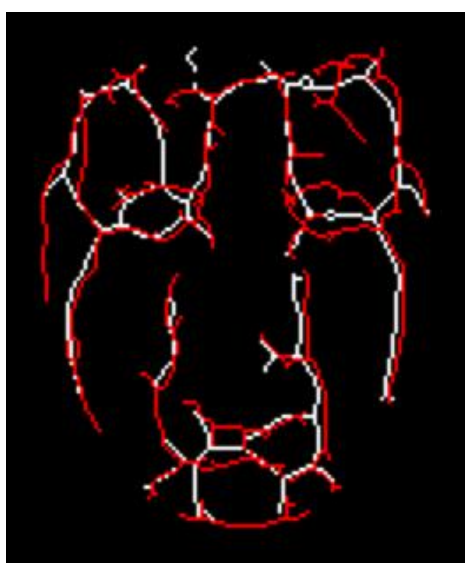

(b)

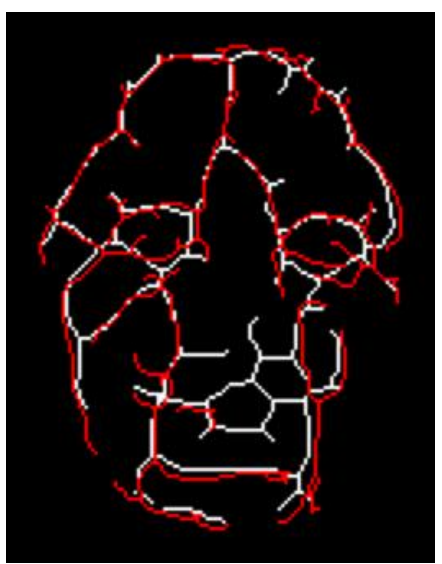

(c)

Fig. 5.8 Overlay of template (white) and signature (red) for positive match (a) subject 12, (b) subject 4 , and (c) subject 6 , similarity values of [0.5252, $0.4986,0.4955]$, respectively

In figure 5.9 we present the overlay of the template and signature for the negative match of subject 4 . In this case figure 5.9a shows the overlay of the template and signature of subject 4 , similarity value is 0.3179 . Figure $5.9 \mathrm{~b}$ shows the overlay for the template of subject 12 to which the signature of subject 4 was negatively matched with a similarity value of 0.3348 . The templates are shown in white and the signatures are shown in red.

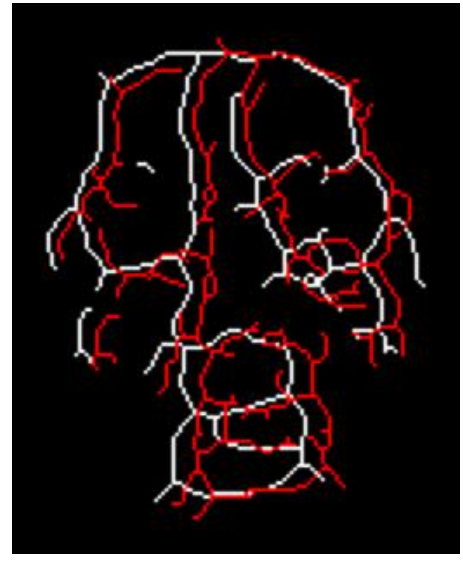

(a)

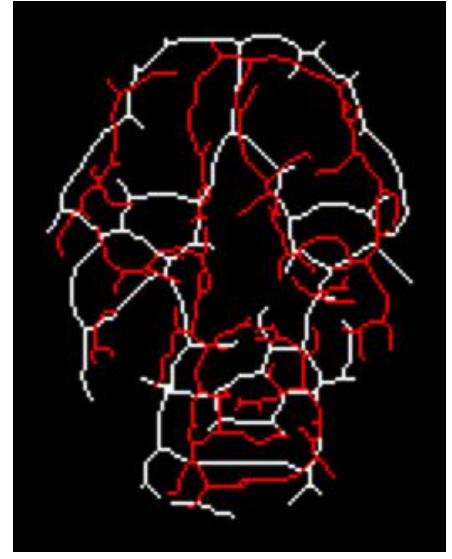

(b)

Fig. 5.9 (a) Subject 4's overlay of its own template and signature, similarity value 0.3179 ,

(b) negative match of the signature of subject 9 to the template of subject 12 with a similarity value of 0.3348 


\subsection{Inter-Subject Results for Diffused Template and Signature}

The similarity measure values presented in this section are given in tables 5.6 -5.10 . The similarity values in these tables were obtained by using diffused templates and diffused signatures. Tables 5.6-5-10 are arranged in the same fashion as the tables in section 5.2.

\subsubsection{Similarity Results for Matching Diffused Templates}

Table 5.6 shows the similarity values $S_{T T}(A \rightarrow B)$, all the diagonal values are equal to one, the thirteen subjects were positively matched using templates with thicker features thus attaining an accuracy rate of $100 \%$.

In figure $5.10 \mathrm{a}$ we present the overlay of diffused template for subject 1 with its own template, and in figure $5.10 \mathrm{~b}$ we present the overlay of the diffused template for subject 1 in white and the diffused template for subject 2 in red.

Table 5.6 Similarity values for $S_{T T}(A \rightarrow B)$, where $A$ and $B$ are diffused templates. Accuracy $100 \%$

\begin{tabular}{|l|l|l|l|l|l|l|}
\hline & Subj01 & Subj02 & Subj03 & Subj04 & Subj05 & Subj06 \\
\hline T-Subj01 & 1.0000 & 0.3532 & 0.4230 & 0.3506 & 0.3952 & 0.4204 \\
\hline T-Subj02 & 0.2562 & 1.0000 & 0.2498 & 0.2821 & 0.3150 & 0.2943 \\
\hline T-Subj03 & 0.4169 & 0.2865 & 1.0000 & 0.3052 & 0.3008 & 0.3407 \\
\hline T-Subj04 & 0.3514 & 0.3411 & 0.3135 & 1.0000 & 0.3698 & 0.3982 \\
\hline T-Subj05 & 0.3694 & 0.3490 & 0.3196 & 0.3664 & 1.0000 & 0.4154 \\
\hline T-Subj06 & 0.4412 & 0.3911 & 0.3791 & 0.4069 & 0.4400 & 1.0000 \\
\hline T-Subj07 & 0.3723 & 0.3504 & 0.3938 & 0.3666 & 0.3529 & 0.3877 \\
\hline T-Subj08 & 0.4411 & 0.3844 & 0.3630 & 0.3881 & 0.3689 & 0.4154 \\
\hline T-Subj09 & 0.3146 & 0.3422 & 0.2890 & 0.3840 & 0.3510 & 0.3329 \\
\hline T-Subj10 & 0.3376 & 0.3629 & 0.3764 & 0.3233 & 0.2903 & 0.3255 \\
\hline T-Subj11 & 0.2642 & 0.3795 & 0.2280 & 0.2281 & 0.2942 & 0.2593 \\
\hline T-Subj12 & 0.4240 & 0.3140 & 0.3902 & 0.3325 & 0.3590 & 0.3697 \\
\hline T-Subj13 & 0.3180 & 0.3457 & 0.2824 & 0.3884 & 0.2920 & 0.3182 \\
\hline
\end{tabular}




\begin{tabular}{|l|l|l|l|l|l|l|l|}
\hline & Subj07 & Subj08 & Subj09 & Subj10 & Subj11 & Subj12 & Subj13 \\
\hline T-Subj01 & 0.2914 & 0.4354 & 0.3522 & 0.3812 & 0.3412 & 0.4019 & 0.3281 \\
\hline T-Subj02 & 0.2342 & 0.3182 & 0.3163 & 0.3572 & 0.4319 & 0.2585 & 0.3067 \\
\hline T-Subj03 & 0.3198 & 0.3150 & 0.2998 & 0.3860 & 0.2894 & 0.3421 & 0.2648 \\
\hline T-Subj04 & 0.3014 & 0.3899 & 0.4014 & 0.3346 & 0.3184 & 0.3152 & 0.3845 \\
\hline T-Subj05 & 0.2464 & 0.3546 & 0.3827 & 0.3041 & 0.3546 & 0.3320 & 0.2879 \\
\hline T-Subj06 & 0.3167 & 0.4213 & 0.4056 & 0.3727 & 0.3554 & 0.3690 & 0.3293 \\
\hline T-Subj07 & 1.0000 & 0.3915 & 0.3867 & 0.3827 & 0.3939 & 0.3866 & 0.3940 \\
\hline T-Subj08 & 0.3472 & 1.0000 & 0.3560 & 0.3590 & 0.3004 & 0.3208 & 0.4042 \\
\hline T-Subj09 & 0.2836 & 0.2983 & 1.0000 & 0.3107 & 0.4108 & 0.3400 & 0.2870 \\
\hline T-Subj10 & 0.3109 & 0.3346 & 0.3139 & 1.0000 & 0.4003 & 0.3268 & 0.3008 \\
\hline T-Subj11 & 0.2299 & 0.2179 & 0.2762 & 0.3111 & 1.0000 & 0.2197 & 0.1767 \\
\hline T-Subj12 & 0.3191 & 0.3204 & 0.3571 & 0.3585 & 0.3040 & 1.0000 & 0.3657 \\
\hline T-Subj13 & 0.3414 & 0.3780 & 0.3190 & 0.3097 & 0.2769 & 0.3567 & 1.0000 \\
\hline
\end{tabular}

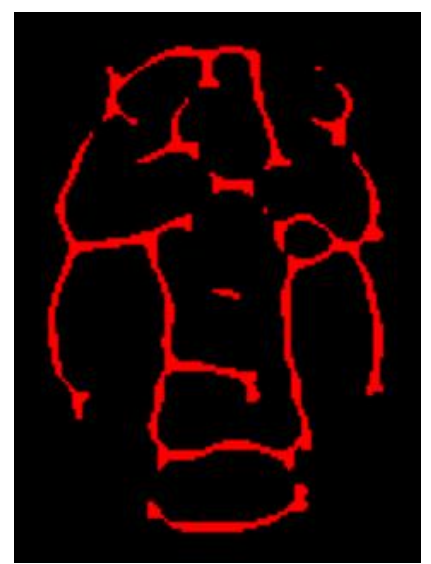

(a)

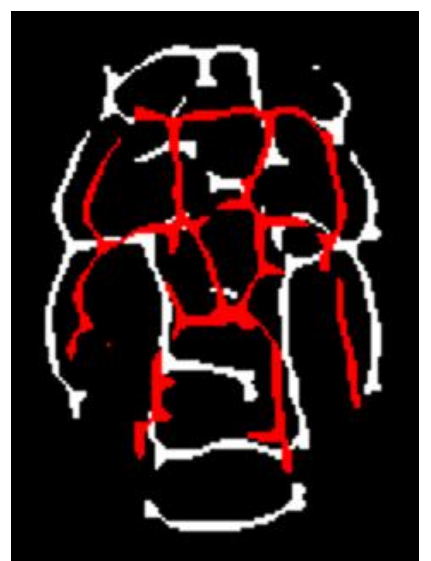

(b)

Fig. 5.10 Overlay of diffused template of (a) subject 1 to its own template and (b) subject 1 template (white) to the template of subject 2 (red)

\subsubsection{Similarity Results for Matching Diffused First Signature Set to Templates}

Table 5.7 we have listed the similarity values for $S_{S 1 T}(A \rightarrow B)$, matching the first set of diffused signatures to the diffused templates. The diagonal values in this table range from 0.3527 to 0.7569 . Twelve subjects were correctly matched, subjects $1-12$; subject 13 was negatively matched to subject 12 . We obtained an accuracy rate of $92.31 \%$. 
Table 5.7 Similarity values $S_{S 1 T}(A \rightarrow B)$, where $A$ is a diffused signature, and $B$ is a diffused template. Accuracy $92.31 \%$

\begin{tabular}{|l|l|l|l|l|l|l|}
\hline & Subj01 & Subj02 & Subj03 & Subj04 & Subj05 & Subj06 \\
\hline T-Subj01 & 0.6006 & 0.3467 & 0.4189 & 0.3641 & 0.3924 & 0.3801 \\
\hline T-Subj02 & 0.2601 & 0.5091 & 0.2446 & 0.2461 & 0.2752 & 0.2643 \\
\hline T-Subj03 & 0.3764 & 0.3331 & 0.5997 & 0.3190 & 0.3383 & 0.3379 \\
\hline T-Subj04 & 0.3214 & 0.3302 & 0.3385 & 0.6466 & 0.4121 & 0.3711 \\
\hline T-Subj05 & 0.3334 & 0.3287 & 0.2875 & 0.3721 & 0.5044 & 0.4134 \\
\hline T-Subj06 & 0.3727 & 0.3776 & 0.3646 & 0.4263 & 0.4567 & 0.6407 \\
\hline T-Subj07 & 0.3742 & 0.3390 & 0.3696 & 0.3598 & 0.3625 & 0.3863 \\
\hline T-Subj08 & 0.3839 & 0.3473 & 0.3489 & 0.4132 & 0.3675 & 0.4100 \\
\hline T-Subj09 & 0.2659 & 0.3433 & 0.2726 & 0.3512 & 0.2974 & 0.3361 \\
\hline T-Subj10 & 0.3333 & 0.3534 & 0.3796 & 0.3166 & 0.3293 & 0.3121 \\
\hline T-Subj11 & 0.2264 & 0.3551 & 0.2436 & 0.1887 & 0.2748 & 0.2533 \\
\hline T-Subj12 & 0.4331 & 0.3391 & 0.3286 & 0.3737 & 0.3694 & 0.3763 \\
\hline T-Subj13 & 0.3047 & 0.3310 & 0.3288 & 0.3375 & 0.3584 & 0.3280 \\
\hline
\end{tabular}

\begin{tabular}{|l|l|l|l|l|l|l|l|}
\hline & Subj07 & Subj08 & Subj09 & Subj10 & Subj11 & Subj12 & Subj13 \\
\hline T-Subj01 & 0.2786 & 0.3354 & 0.3528 & 0.3322 & 0.2944 & 0.3915 & 0.3422 \\
\hline T-Subj02 & 0.1959 & 0.2466 & 0.2357 & 0.3650 & 0.4263 & 0.2320 & 0.1922 \\
\hline T-Subj03 & 0.3128 & 0.3109 & 0.2950 & 0.3467 & 0.3141 & 0.3176 & 0.2972 \\
\hline T-Subj04 & 0.3076 & 0.3553 & 0.3793 & 0.2982 & 0.3157 & 0.3023 & 0.2843 \\
\hline T-Subj05 & 0.2339 & 0.3423 & 0.3857 & 0.3193 & 0.3165 & 0.3335 & 0.3196 \\
\hline T-Subj06 & 0.2990 & 0.3437 & 0.4243 & 0.3664 & 0.3739 & 0.3462 & 0.3242 \\
\hline T-Subj07 & 0.5144 & 0.3980 & 0.3780 & 0.3827 & 0.3805 & 0.3783 & 0.3544 \\
\hline T-Subj08 & 0.3073 & 0.5807 & 0.3407 & 0.3379 & 0.3360 & 0.3143 & 0.3667 \\
\hline T-Subj09 & 0.2393 & 0.2914 & 0.5295 & 0.2905 & 0.3104 & 0.3507 & 0.2688 \\
\hline T-Subj10 & 0.2888 & 0.3036 & 0.2752 & 0.5319 & 0.3463 & 0.2994 & 0.3060 \\
\hline T-Subj11 & 0.1536 & 0.2242 & 0.2930 & 0.2315 & 0.4332 & 0.2032 & 0.2166 \\
\hline T-Subj12 & 0.2853 & 0.3983 & 0.3721 & 0.3723 & 0.3126 & 0.7569 & 0.3764 \\
\hline T-Subj13 & 0.3286 & 0.3646 & 0.3453 & 0.3050 & 0.2886 & 0.3107 & 0.3527 \\
\hline
\end{tabular}

In figure 5.11 we show the overlay of the diffused template and signature corresponding to a positive match for subjects 12,4 , and 6 ; these subjects had the highest similarity values in table $5.7,[0.7569,0.6466,0.6407]$ respectively. The templates are shown in white and the signatures are shown in red. 


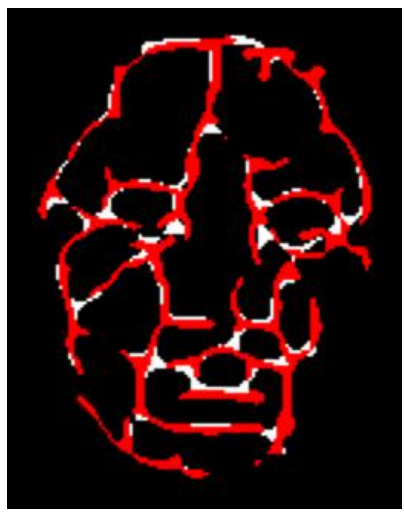

(a)

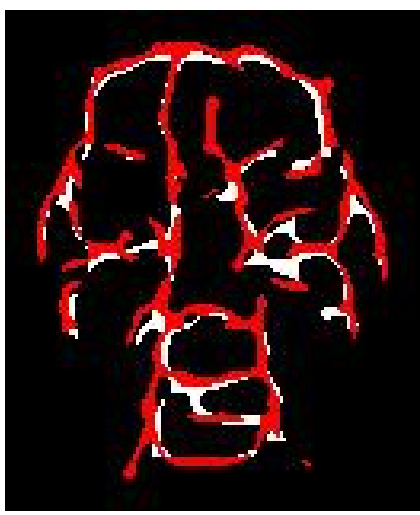

(b)

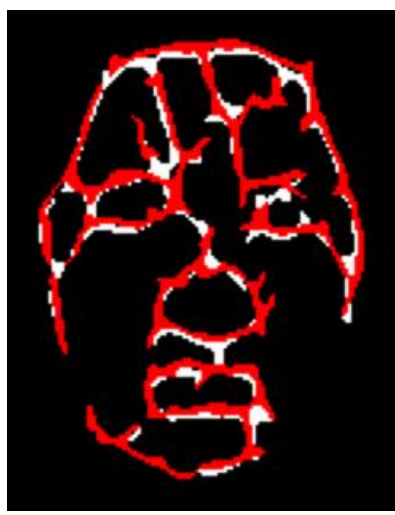

(c)

Fig. 5.11 Overlay of diffused template (white) and signature (red) for positive match (a) subject 12 , (b) subject 4 , and (c) subject 6 , similarity values [0.7569, 0.6466, 0.6407] respectively

In figure 5.12 we present the overlay of the diffused template and signature for the negative match of subject 13 . Figure 5.12a shows the overlay of the diffused template and signature of subject 13 , similarity value is 0.3527 . Figure $5.12 \mathrm{~b}$ shows the overlay for the diffused template of subject 12 to which the diffused signature of subject 4 was negatively matched, similarity value of 0.3764 . The templates are shown in white and the signatures are shown in red.

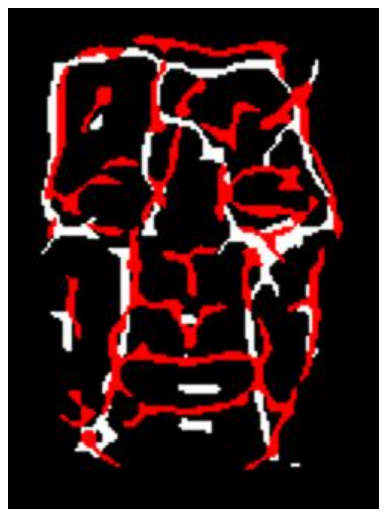

(a)

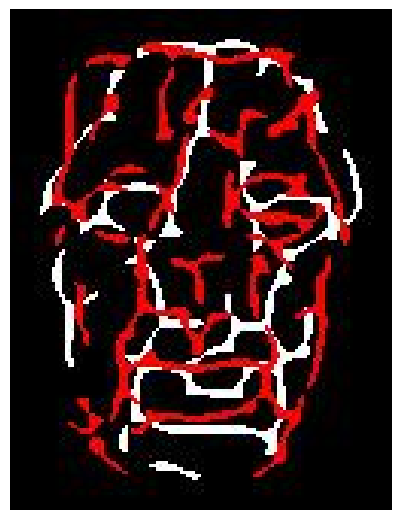

(b)

Fig. 5.12 (a) Subject 13 overlay of its own diffused template and signature, similarity value 0.3527 , (b) negative match subject 13 to the template of subject 12 , similarity value of 0.3764 . 


\subsubsection{Similarity Results for Matching Diffused Second Signature Set to Templates}

In table 5.8 we have listed the similarity values for $S_{S 2 T}(A \rightarrow B)$, matching the second set of diffused signatures to the diffused templates. The diagonal values in this table range from 0.3081 to 0.7356 . In this case subjects $1,2,4-8$, and $10-13$ are positively matched, whereas subjects 3 and 9 are matched to subjects 12 and 6 respectively. We obtained an accuracy rate of $84.61 \%$.

In figure 5.13 we show the overlay of the diffused template and signature corresponding to a positive match for subjects 12,6 , and 1; these subjects had the highest similarity values in table 5.8, $[0.7356,0.7129,0.5479]$ respectively. The templates are shown in white and the signatures are shown in red.

Table 5.8 Similarity values for $S_{S 2 T}(A \rightarrow B)$, where $A$ is the diffused signature, and $B$ is a diffused template. Accuracy $84.61 \%$

\begin{tabular}{|l|l|l|l|l|l|l|}
\hline & Subj01 & Subj02 & Subj03 & Subj04 & Subj05 & Subj06 \\
\hline T-Subj01 & 0.5479 & 0.3037 & 0.3967 & 0.3602 & 0.4016 & 0.3771 \\
\hline T-Subj02 & 0.2910 & 0.4615 & 0.2569 & 0.2362 & 0.2670 & 0.2838 \\
\hline T-Subj03 & 0.3497 & 0.2813 & 0.3163 & 0.2828 & 0.3175 & 0.3456 \\
\hline T-Subj04 & 0.2919 & 0.3537 & 0.3535 & 0.5368 & 0.3788 & 0.3930 \\
\hline T-Subj05 & 0.3236 & 0.3517 & 0.3217 & 0.3596 & 0.5320 & 0.4322 \\
\hline T-Subj06 & 0.4042 & 0.3837 & 0.3622 & 0.4235 & 0.4024 & 0.7129 \\
\hline T-Subj07 & 0.3986 & 0.3333 & 0.3937 & 0.3682 & 0.3598 & 0.4061 \\
\hline T-Subj08 & 0.4395 & 0.3290 & 0.3677 & 0.4286 & 0.3453 & 0.4008 \\
\hline T-Subj09 & 0.3129 & 0.3270 & 0.3215 & 0.3338 & 0.3329 & 0.3226 \\
\hline T-Subj10 & 0.3652 & 0.3815 & 0.3010 & 0.3108 & 0.3065 & 0.2958 \\
\hline T-Subj11 & 0.2182 & 0.3181 & 0.2449 & 0.1851 & 0.2702 & 0.2251 \\
\hline T-Subj12 & 0.3927 & 0.3301 & $\mathbf{0 . 4 2 4 9}$ & 0.3714 & 0.3749 & 0.3889 \\
\hline T-Subj13 & 0.3373 & 0.3269 & 0.3227 & 0.3227 & 0.2978 & 0.3015 \\
\hline
\end{tabular}




\begin{tabular}{|l|l|l|l|l|l|l|l|}
\hline & Subj07 & Subj08 & Subj09 & Subj10 & Subj11 & Subj12 & Subj13 \\
\hline T-Subj01 & 0.2746 & 0.3270 & 0.2714 & 0.3011 & 0.3188 & 0.4101 & 0.3288 \\
\hline T-Subj02 & 0.2439 & 0.2379 & 0.3624 & 0.3391 & 0.4078 & 0.2381 & 0.2617 \\
\hline T-Subj03 & 0.3151 & 0.3280 & 0.2416 & 0.3507 & 0.3329 & 0.3075 & 0.3453 \\
\hline T-Subj04 & 0.3115 & 0.3617 & 0.3005 & 0.3147 & 0.2986 & 0.3187 & 0.3388 \\
\hline T-Subj05 & 0.2492 & 0.3454 & 0.3129 & 0.3183 & 0.3440 & 0.3472 & 0.3700 \\
\hline T-Subj06 & 0.3181 & 0.3418 & 0.3776 & 0.3586 & 0.3706 & 0.3684 & 0.2996 \\
\hline T-Subj07 & 0.5363 & 0.4032 & 0.3421 & 0.3940 & 0.3812 & 0.3714 & 0.3960 \\
\hline T-Subj08 & 0.3321 & 0.5358 & 0.3013 & 0.3402 & 0.3210 & 0.3255 & 0.4041 \\
\hline T-Subj09 & 0.2642 & 0.2815 & 0.3081 & 0.2925 & 0.3163 & 0.3379 & 0.3253 \\
\hline T-Subj10 & 0.3181 & 0.2916 & 0.2980 & 0.5357 & 0.3857 & 0.2819 & 0.3001 \\
\hline T-Subj11 & 0.1778 & 0.2224 & 0.2904 & 0.2375 & 0.4328 & 0.2250 & 0.2124 \\
\hline T-Subj12 & 0.3025 & 0.3899 & 0.3237 & 0.3919 & 0.3244 & 0.7356 & 0.3459 \\
\hline T-Subj13 & 0.3333 & 0.3686 & 0.2901 & 0.2800 & 0.3044 & 0.3218 & 0.4311 \\
\hline
\end{tabular}

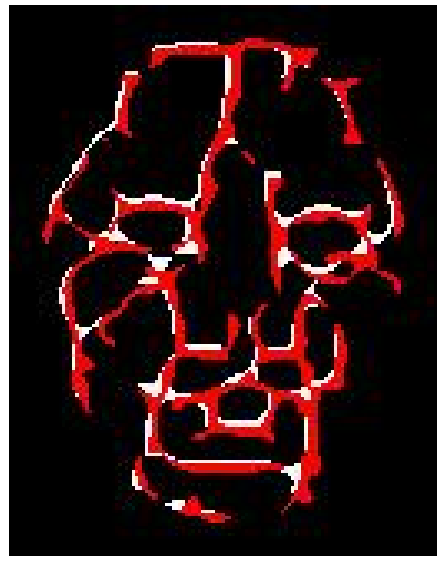

(a)

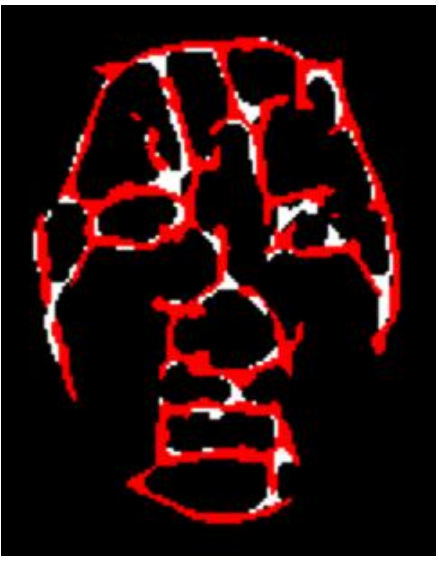

(b)

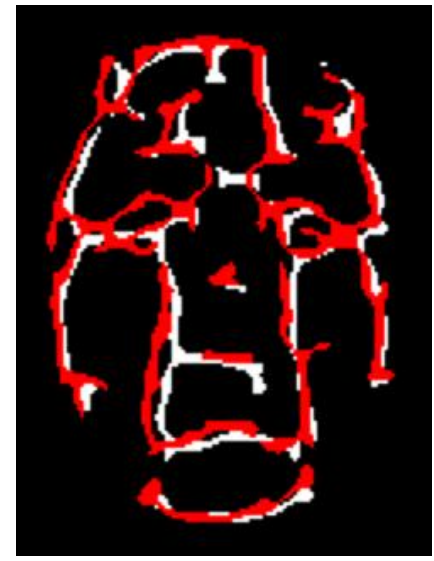

(c)

Fig. 5.13 Overlay of diffused template (white) and signature (red) for positive match (a) subject 12 , (b) subject 6 , and (c) subject 1 , with similarity values [0.7356, 0.7129, 0.5479] respectively

In figure 5.14 we present the overlay of the diffused template and signature for the negative match of subject 3 . Figure 5.14 a shows the overlay of the diffused template and signature of subject 3 , similarity value is 0.3163 . Figure $5.14 \mathrm{~b}$ shows the overlay for the diffused template of subject 12 to which the diffused signature of subject 3 was negatively 
matched with similarity value of 0.4249 . The templates are shown in white and the signatures are shown in red.

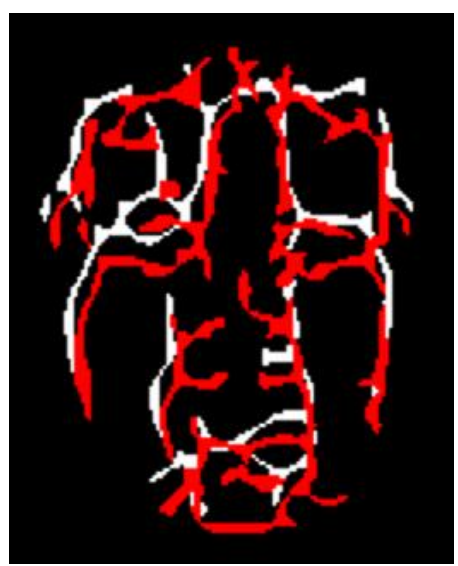

(a)

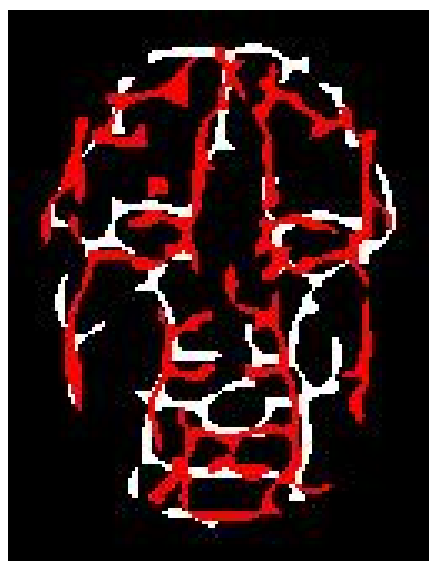

(b)

Fig. 5.14 (a) Subject 3 overlay of its own diffused template and signature, similarity value 0.3163 , (b) negative match subject 3 to the template of subject 12 , similarity value of 0.4249

\subsubsection{Similarity Results for Matching Diffused Third Signature Set to Templates}

In table 5.9 we have listed the similarity values for $S_{S 3 T}(A \rightarrow B)$, matching the third set of diffused signatures to the diffused templates. The diagonal values range from 0.3026-0.6799. In this case subject 9 was matched to subject 7, the other 12 subjects were matched correctly. We obtained an accuracy rate of $92.31 \%$.

In figure 5.15 we show the overlay of the diffused template and signature corresponding to a positive match for subjects 12,5 , and 6 ; these subjects had the highest similarity values in table $5.9,[0.6799,0.5787,0.5733]$ respectively. The templates are shown in white and the signatures are shown in red. 
Table 5.9 Similarity values $S_{S 3 T}(A \rightarrow B)$, where $A$ is a diffused signature, and $B$ is a diffused template. Accuracy $92.31 \%$.

\begin{tabular}{|l|l|l|l|l|l|l|}
\hline & Subj01 & Subj02 & Subj03 & Subj04 & Subj05 & Subj06 \\
\hline T-Subj01 & 0.5273 & 0.3080 & 0.3751 & 0.3343 & 0.3552 & 0.3440 \\
\hline T-Subj02 & 0.3162 & 0.5159 & 0.2539 & 0.2770 & 0.3247 & 0.3410 \\
\hline T-Subj03 & 0.3035 & 0.2741 & 0.4769 & 0.3426 & 0.2942 & 0.3028 \\
\hline T-Subj04 & 0.3409 & 0.3278 & 0.3407 & 0.5922 & 0.3240 & 0.3797 \\
\hline T-Subj05 & 0.4136 & 0.3134 & 0.2931 & 0.3269 & 0.5787 & 0.3579 \\
\hline T-Subj06 & 0.4330 & 0.3669 & 0.3524 & 0.3783 & 0.3960 & 0.5733 \\
\hline T-Subj07 & 0.3690 & 0.3313 & 0.4063 & 0.3687 & 0.3796 & 0.3795 \\
\hline T-Subj08 & 0.3703 & 0.3452 & 0.3316 & 0.4115 & 0.3305 & 0.3860 \\
\hline T-Subj09 & 0.3641 & 0.3033 & 0.3044 & 0.3397 & 0.3526 & 0.3383 \\
\hline T-Subj10 & 0.3037 & 0.3598 & 0.3185 & 0.3626 & 0.2726 & 0.3181 \\
\hline T-Subj11 & 0.2499 & 0.3403 & 0.1983 & 0.2494 & 0.3011 & 0.2722 \\
\hline T-Subj12 & 0.4248 & 0.3295 & 0.4136 & 0.3155 & 0.3390 & 0.3634 \\
\hline T-Subj13 & 0.2911 & 0.3203 & 0.2822 & 0.3691 & 0.2890 & 0.3307 \\
\hline
\end{tabular}

\begin{tabular}{|l|l|l|l|l|l|l|l|}
\hline & Subj07 & Subj08 & Subj09 & Subj10 & Subj11 & Subj12 & Subj13 \\
\hline T-Subj01 & 0.2791 & 0.3264 & 0.2749 & 0.2835 & 0.3088 & 0.3643 & 0.2720 \\
\hline T-Subj02 & 0.2131 & 0.2538 & 0.1914 & 0.2730 & 0.3541 & 0.2576 & 0.2249 \\
\hline T-Subj03 & 0.3055 & 0.3086 & 0.2381 & 0.3717 & 0.3052 & 0.3091 & 0.2913 \\
\hline T-Subj04 & 0.2771 & 0.3454 & 0.2815 & 0.3031 & 0.3000 & 0.3280 & 0.3138 \\
\hline T-Subj05 & 0.2351 & 0.2831 & 0.2603 & 0.2962 & 0.3354 & 0.3674 & 0.2506 \\
\hline T-Subj06 & 0.2525 & 0.3475 & 0.2720 & 0.3399 & 0.3320 & 0.3759 & 0.2821 \\
\hline T-Subj07 & 0.4874 & 0.3745 & $\mathbf{0 . 4 0 7 2}$ & 0.3672 & 0.3601 & 0.3766 & 0.4055 \\
\hline T-Subj08 & 0.3209 & 0.4684 & 0.2750 & 0.3348 & 0.3032 & 0.3110 & 0.3241 \\
\hline T-Subj09 & 0.2934 & 0.3126 & 0.3026 & 0.2748 & 0.3246 & 0.3391 & 0.2597 \\
\hline T-Subj10 & 0.2821 & 0.2983 & 0.2196 & 0.4373 & 0.3735 & 0.2896 & 0.2876 \\
\hline T-Subj11 & 0.1873 & 0.2742 & 0.1685 & 0.2985 & 0.4449 & 0.1854 & 0.2032 \\
\hline T-Subj12 & 0.3529 & 0.3834 & 0.3322 & 0.3713 & 0.3185 & 0.6799 & 0.3330 \\
\hline T-Subj13 & 0.3438 & 0.3118 & 0.2644 & 0.2823 & 0.2862 & 0.3271 & 0.4331 \\
\hline
\end{tabular}




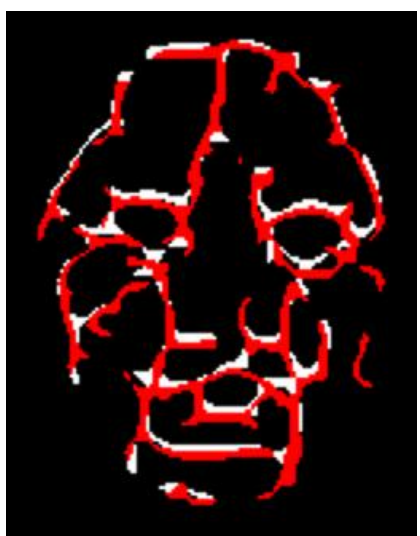

(a)

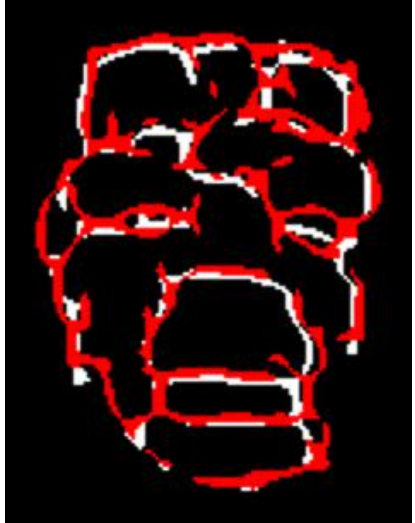

(b)

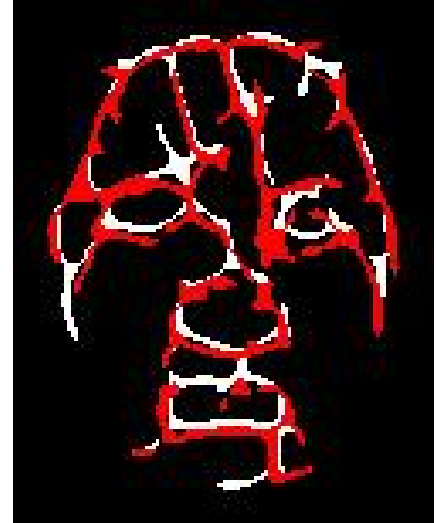

(c)

Fig. 5.15 Overlay of diffused template (white) and signature (red) for positive match (a) subject 12 , (b) subject 5 , and (c) subject 6 , with similarity values [0.6799, 0.5787, 0.5733] respectively

In figure 5.16 we present the overlay of the diffused template and signature for the negative match of subject 9. Figure 5.16a shows the overlay of the diffused template and signature of subject 9 , similarity value is 0.3026 . Figure $5.16 \mathrm{~b}$ shows the overlay for the diffused template of subject 7 to which the diffused signature of subject 9 was negatively matched with similarity value of 0.4072 . The templates are shown in white and the signatures are shown in red.

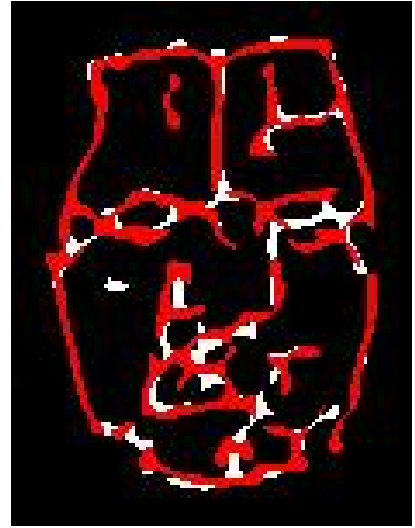

(a)

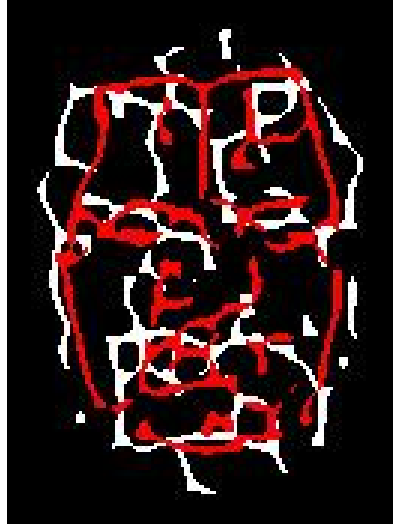

(b)

Fig. 5.16 (a) Subject 9 overlay of its own diffused template and signature, similarity value $0.3026,(\mathrm{~b})$ negative match subject 9 to the template of subject 7 , similarity value of 0.4072 


\subsubsection{Similarity Results for Matching Diffused Fourth Signature Set to Templates}

In table 5.10 the similarity values for $S_{S 4 T}(A \rightarrow B)$ are given. The diagonal values range from $0.3895-0.6240$. In this case subject 4 was matched to subject 6 ; the other 12 subjects were positively matched. We obtained an accuracy rate of $92.31 \%$.

Table 5.10 Similarity values $S_{S 4 T}(A \rightarrow B), A$ is diffused signature, and $B$ is a diffused template. Accuracy $92.31 \%$

\begin{tabular}{|l|l|l|l|l|l|l|}
\hline & Subj01 & Subj02 & Subj03 & Subj04 & Subj05 & Subj06 \\
\hline T-Subj01 & 0.5436 & 0.3528 & 0.3920 & 0.3359 & 0.3789 & 0.3931 \\
\hline T-Subj02 & 0.2715 & 0.5279 & 0.2620 & 0.2445 & 0.2570 & 0.3338 \\
\hline T-Subj03 & 0.3469 & 0.3170 & 0.5691 & 0.3157 & 0.3016 & 0.2760 \\
\hline T-Subj04 & 0.3631 & 0.3380 & 0.3060 & 0.3895 & 0.3469 & 0.3445 \\
\hline T-Subj05 & 0.4479 & 0.3510 & 0.2899 & 0.3982 & 0.5674 & 0.3589 \\
\hline T-Subj06 & 0.4403 & 0.4075 & 0.3687 & 0.4389 & 0.4519 & 0.6362 \\
\hline T-Subj07 & 0.3750 & 0.3325 & 0.3705 & 0.3417 & 0.3390 & 0.3898 \\
\hline T-Subj08 & 0.4009 & 0.3545 & 0.3387 & 0.3571 & 0.3357 & 0.3989 \\
\hline T-Subj09 & 0.3238 & 0.3383 & 0.2336 & 0.3332 & 0.3155 & 0.3500 \\
\hline T-Subj10 & 0.3243 & 0.3628 & 0.3097 & 0.3406 & 0.2624 & 0.3146 \\
\hline T-Subj11 & 0.2404 & 0.3506 & 0.2245 & 0.2825 & 0.2646 & 0.2228 \\
\hline T-Subj12 & 0.4138 & 0.3274 & 0.3602 & 0.4146 & 0.3419 & 0.3737 \\
\hline T-Subj13 & 0.2821 & 0.3161 & 0.2784 & 0.3247 & 0.2616 & 0.3346 \\
\hline
\end{tabular}

\begin{tabular}{|l|l|l|l|l|l|l|l|}
\hline & Subj07 & Subj08 & Subj09 & Subj10 & Subj11 & Subj12 & Subj13 \\
\hline T-Subj01 & 0.3697 & 0.3691 & 0.3896 & 0.3053 & 0.3302 & 0.3936 & 0.2750 \\
\hline T-Subj02 & 0.2981 & 0.2316 & 0.2860 & 0.3011 & 0.4065 & 0.2359 & 0.2154 \\
\hline T-Subj03 & 0.2817 & 0.3423 & 0.2938 & 0.3600 & 0.2827 & 0.3236 & 0.2894 \\
\hline T-Subj04 & 0.3010 & 0.3705 & 0.3576 & 0.3627 & 0.3436 & 0.3369 & 0.3236 \\
\hline T-Subj05 & 0.3619 & 0.3759 & 0.4230 & 0.2732 & 0.3623 & 0.3176 & 0.2340 \\
\hline T-Subj06 & 0.3478 & 0.3455 & 0.3952 & 0.3716 & 0.3979 & 0.3824 & 0.2975 \\
\hline T-Subj07 & 0.4542 & 0.3669 & 0.3783 & 0.3768 & 0.4036 & 0.3777 & 0.4139 \\
\hline T-Subj08 & 0.3240 & 0.4504 & 0.3373 & 0.3571 & 0.3293 & 0.3542 & 0.3224 \\
\hline T-Subj09 & 0.3520 & 0.3198 & 0.5058 & 0.2960 & 0.3700 & 0.3454 & 0.2519 \\
\hline T-Subj10 & 0.3122 & 0.2686 & 0.3060 & 0.4933 & 0.3930 & 0.3433 & 0.2709 \\
\hline T-Subj11 & 0.2792 & 0.2000 & 0.2845 & 0.2612 & 0.5327 & 0.2089 & 0.2035 \\
\hline T-Subj12 & 0.3895 & 0.3761 & 0.3769 & 0.3762 & 0.3487 & 0.6240 & 0.3228 \\
\hline T-Subj13 & 0.3354 & 0.3095 & 0.3457 & 0.3200 & 0.2942 & 0.2928 & 0.4338 \\
\hline
\end{tabular}


In figure 5.17 we show the overlay of the diffused template and signature corresponding to a positive match for subjects 6,12 , and 3; these subjects had the highest similarity values in table $5.10,[0.6362,0.6240,0.5691]$ respectively. The templates are shown in white and the signatures are shown in red.

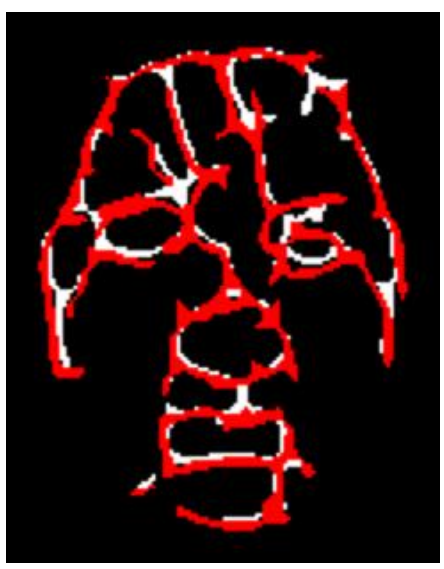

(a)

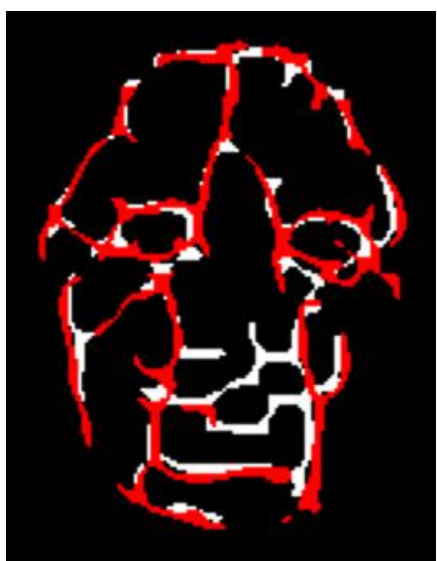

(b)

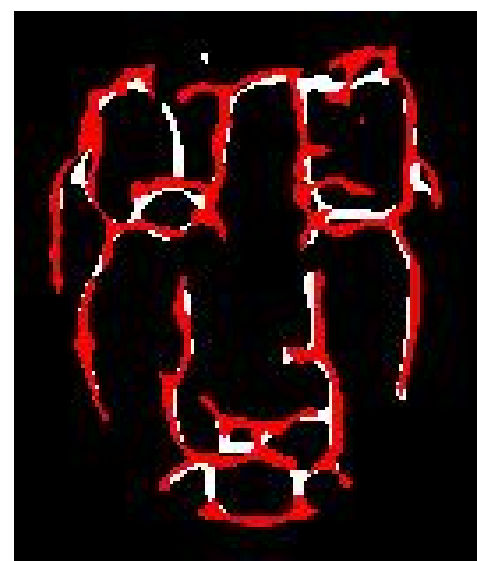

(c)

Fig. 5.17 Overlay of diffused template (white) and signature (red) for positive match (a) subject, (6b) subject 12 , and (c) subject 3 , with similarity values [0.6362, 0.6240, 0.5691] respectively.

In figure 5.18 we present the overlay of the diffused template and signature for the negative match of subject 4 . Figure 5.18 a shows the overlay of the diffused template and signature of subject 4 , similarity value is 0.3895 . Figure $5.18 \mathrm{~b}$ shows the overlay for the diffused template of subject 6 to which the diffused signature of subject 4 was negatively matched with similarity value of 0.4389 . The templates are shown in white and the signatures are shown in red. 


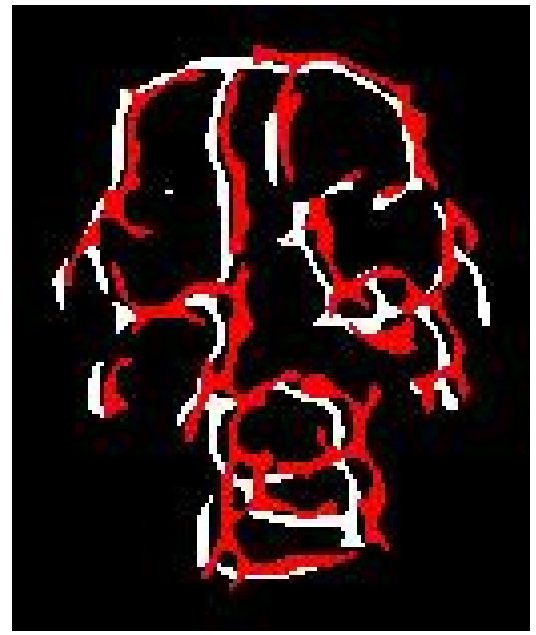

(a)

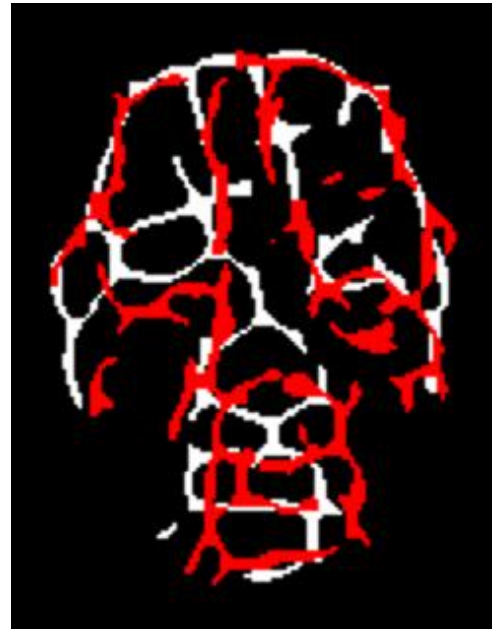

(b)

Fig. 5.18 (a) Subject 4 overlay of its own diffused template and signature, similarity value 0.3895 , (b) negative match of subject 4 signature to the template of subject 6 , similarity value of 0.4389

\subsubsection{Similarity Results for Matching Signatures to Modified Templates}

The results in this section present the similarity accuracies obtained from matching the skeletonized signatures to a modified template. In section 3.2.3 the methodology to obtain a template was explained in detail, in the modified method for this section we use three signatures per subject to obtain the template. In order to match a signature to a template the excluded signature from the template creation process is used as the testing signature, thus we obtained 4 different templates and 4 similarity measure value tables. The images in figure 5.19 show the overlay of the new template (white) and the testing signature (red) for one subject in our database. The label T123-S4 refers to the template being created with signatures 1,2 , and 3 while signature 4 is used as the non-reference image when applying the similarity measure, the rest of the images in figure 5.19 are labeled in a similar fashion. 


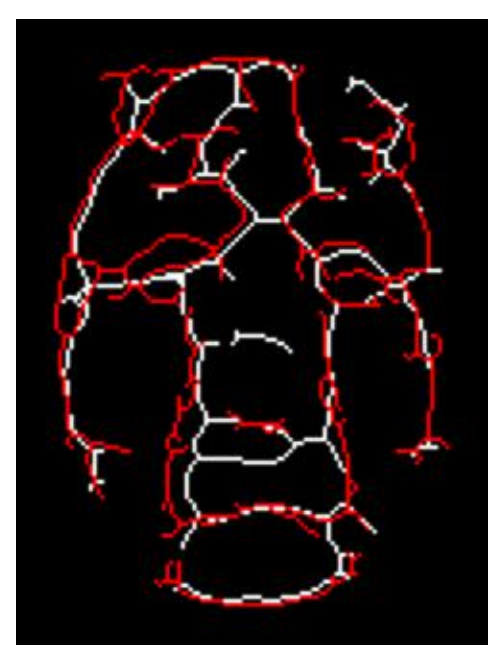

(a) T234-S1

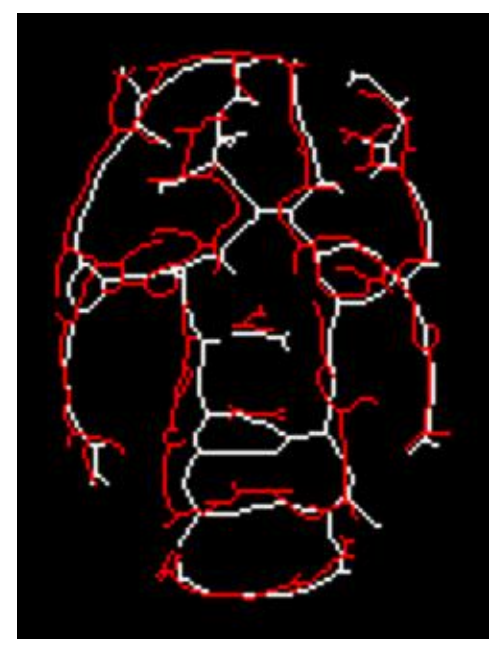

(c) T124-S3

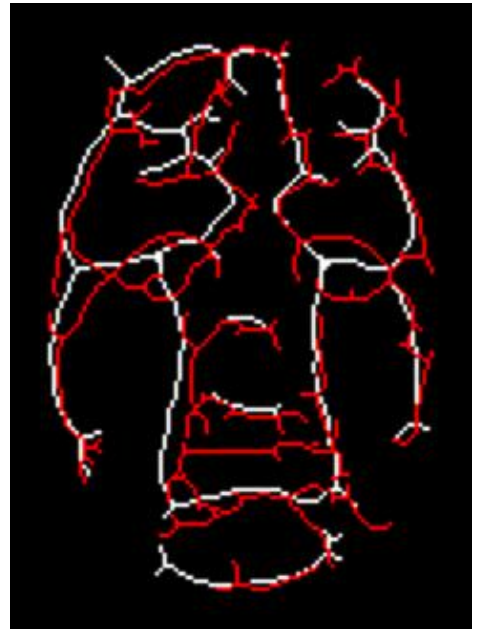

(b) T134-S2

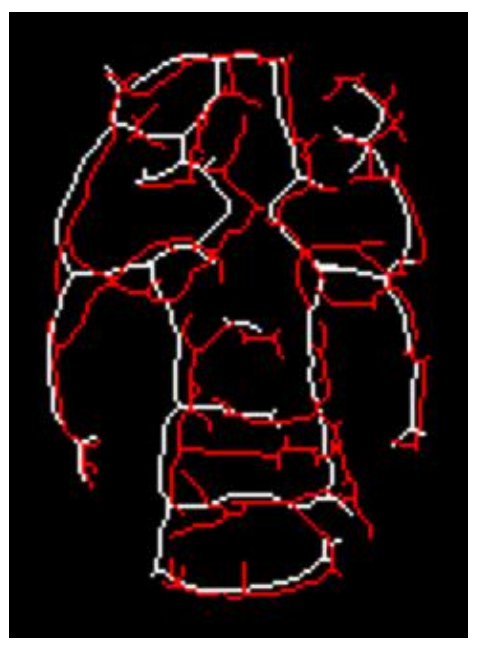

(d) T123-S4

Fig. 5.19 Overlay of signatures and modified templates of subject 1

Note how the modified templates change slightly depending on the signatures used to create it. The accuracy results for this experiment are shown in table 5.11 which also contains the results obtained previously with a template created using four signatures.

Table 5.11 Accuracy for matching signatures to templates created using 3 signatures (Template-3S) and 4 signatures (Template-4S). Templates and signatures are skeletonized.

\begin{tabular}{|l|l|l|l|l|l|}
\hline & S1 & S2 & S3 & S4 & Avg. \\
\hline Template-3S & $92.31 \%$ & $61.54 \%$ & $84.62 \%$ & $92.31 \%$ & $82.69 \%$ \\
\hline Template-4S & $92.31 \%$ & $76.92 \%$ & $92.31 \%$ & $92.31 \%$ & $88.46 \%$ \\
\hline
\end{tabular}


The accuracy values decreased on average by $5.77 \%$ when three signatures were used to create the template. The accuracy dropped most significantly when using signatures 1,3 , and 4 to create the template and the signature set 2 was used for matching. The drop in the accuracy was expected as the information of the testing signature is no longer contained in the template.

The similarity measure was also applied to templates thickened using a rotationally symmetric Gaussian low-pass filter of size 2 and with a 1.5 standard deviation. Using this filter allows us to thicken the template to a width of 2 pixels. The templates created using 3 and 4 signatures were thickened using this Gaussian filter, the testing signature sets were left in their skeletonized form. Figure 5.20 shows an example of the thicker template (white), using 3 signatures, overlaid with the testing signature (red).

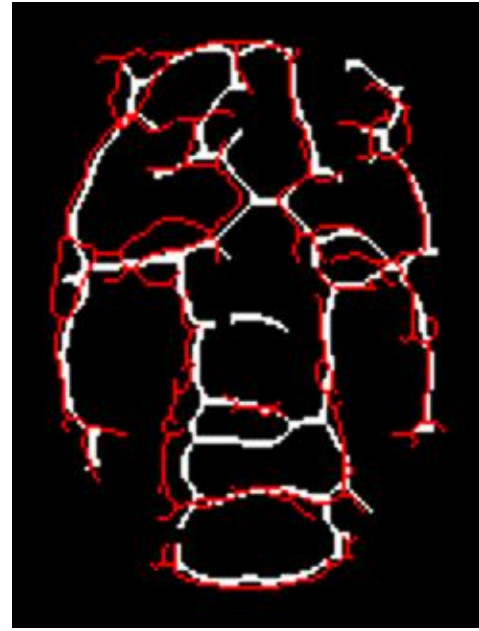

(a) T234-S1

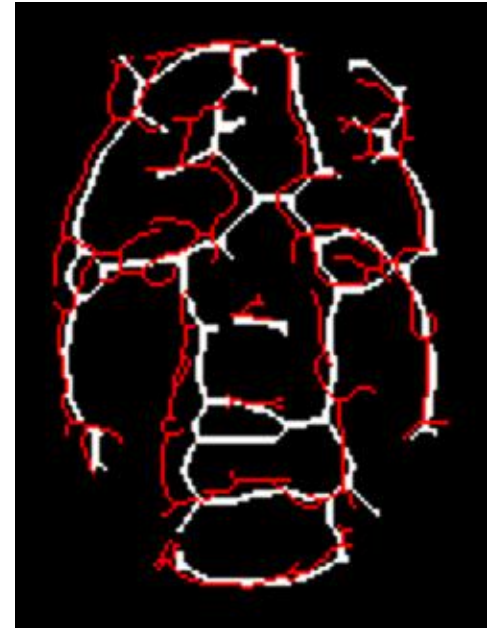

(b) T134-S2 


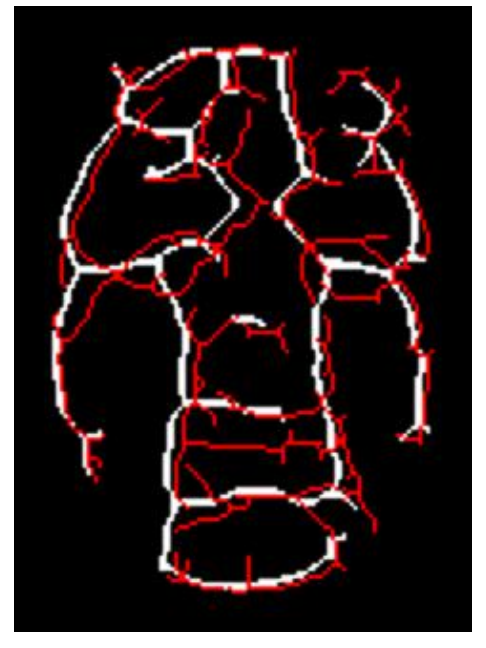

(c) T124-S3

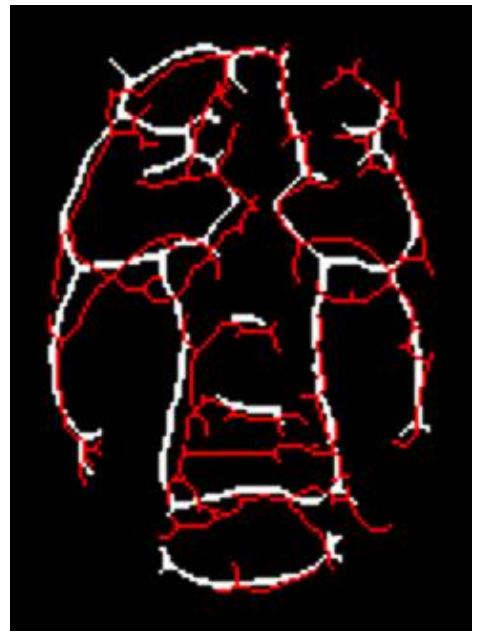

(d) T123-S4

Fig. 5.20 Overlay of two-pixels thick templates and skeletonized signatures for subject 1 .

In figure 5.21 we present an example of a two-pixel thick template (white), created using 4 signatures, overlaid with the testing signature (red). The naming of images 5.21 a-c follow the format $\mathrm{T} 4-\mathrm{S} 1$, in this case $\mathrm{T} 4$ means that the template was created using 4 signatures and S1 denotes that signature 1 was used for testing.

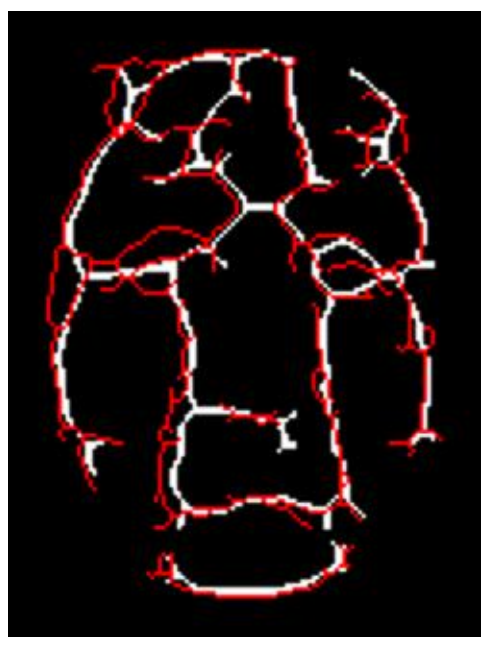

(a) T4-S1

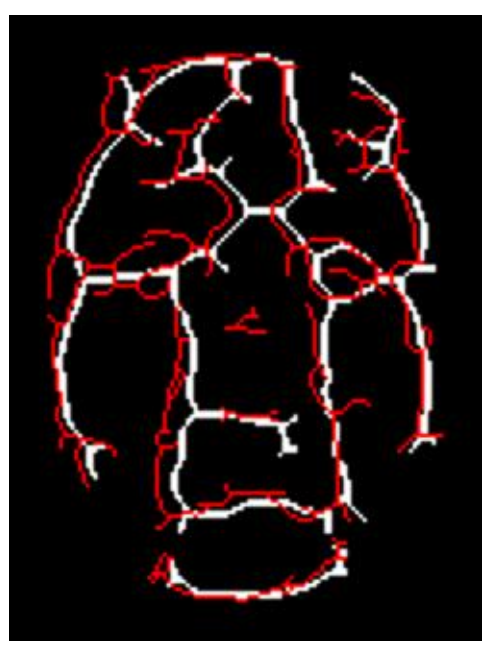

(b) T4-S2 


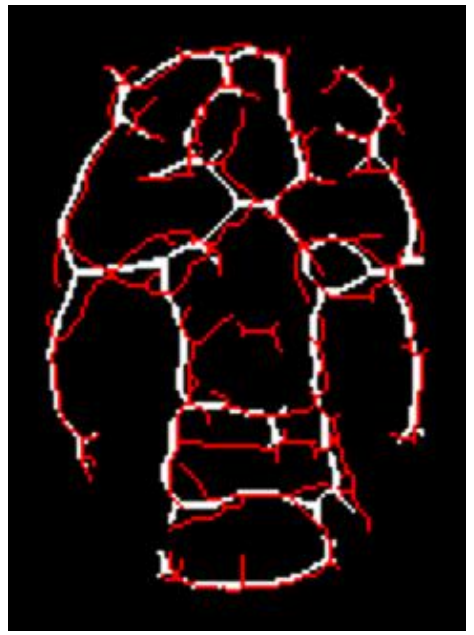

(c) T4-S3

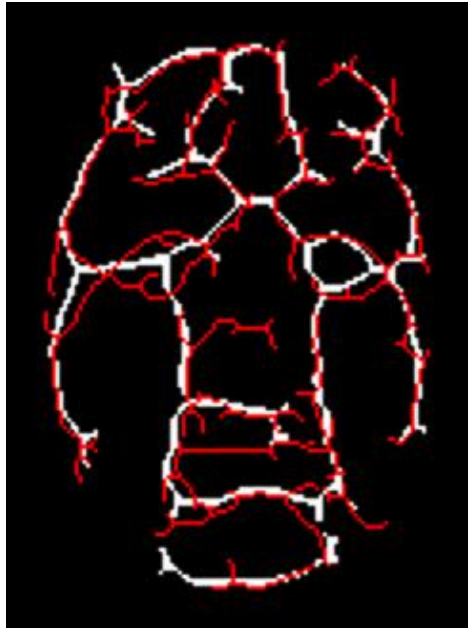

(d) T4-S4

Fig. 5.21 Overlay of two-pixels thick templates and skeletonized signatures for subject 1

The accuracy results obtained for matching each skeletonized signature set to the two-pixels thick templates are shown in table 5.12. The results show a small decrease in the accuracy when the similarity measure is applied to the template created using three signatures. The accuracy in this case was $82.69 \%$, the same accuracy was obtained when both the template and the signature are in their skeletonized form. However the accuracy obtained for the combination of the two-pixel thick template, created using 4 signatures, and the skeletonized signature is $84.62 \%$. There is only a $1.93 \%$ accuracy difference in the results obtained using a two-pixel thick template created with 4 signatures and a two-pixel thick template created with 3 signatures, as it is shown in table 5.12.

Table 5.12 Accuracy values for matching skeletonized signatures to templates that are 2 pixels thick

\begin{tabular}{|l|c|c|c|c|c|}
\hline & S1 & S2 & S3 & S4 & Avg. \\
\hline T-3 Signatures & $92.31 \%$ & $61.54 \%$ & $84.62 \%$ & $92.31 \%$ & $82.69 \%$ \\
\hline T-4 Signatures & $92.31 \%$ & $76.92 \%$ & $76.92 \%$ & $92.31 \%$ & $84.62 \%$ \\
\hline
\end{tabular}




\subsection{Intra-Subject Results}

Intra-subject similarity values are given in tables 5.13 and 5.14 intra-subject results correspond to the values found in the diagonal trace of tables 5.1-5.10. The similarity values in table 5.13 are for skeletonized templates and signatures; corresponding to the diagonal traces found in tables 5.1-5.5. The values shown in table 5.14 are for diffused templates and signatures; corresponding to the diagonal traces found in tables 5.6-5.10. For each signature set the diagonal trace is presented column-wise and ordered by subject number. The template was always the reference image and the signature was the non-reference image. The similarity values leading to a mismatch are in shown in red.

Table 5.13 Intra-subject similarity values. Skeletonized templates and signatures.

\begin{tabular}{|l|c|c|c|c|c|}
\hline & Template & $\begin{array}{c}\text { Signature } \\
\text { Set 1 }\end{array}$ & $\begin{array}{c}\text { Signature } \\
\text { Set 2 }\end{array}$ & $\begin{array}{c}\text { Signature } \\
\text { Set 3 }\end{array}$ & $\begin{array}{c}\text { Signature } \\
\text { Set 4 }\end{array}$ \\
\hline Subj01 & 1.0000 & 0.4957 & 0.4064 & 0.4190 & 0.4280 \\
\hline Subj02 & 1.0000 & 0.4115 & 0.3644 & 0.3898 & 0.4113 \\
\hline Subj03 & 1.0000 & 0.4635 & 0.3001 & 0.3883 & 0.4986 \\
\hline Subj04 & 1.0000 & 0.4916 & 0.4161 & 0.4606 & 0.3179 \\
\hline Subj05 & 1.0000 & 0.3740 & 0.3784 & 0.4502 & 0.4437 \\
\hline Subj06 & 1.0000 & 0.5043 & 0.5806 & 0.4556 & 0.5252 \\
\hline Subj07 & 1.0000 & 0.4178 & 0.4345 & 0.3836 & 0.3828 \\
\hline Subj08 & 1.0000 & 0.4648 & 0.4351 & 0.3771 & 0.3695 \\
\hline Subj09 & 1.0000 & 0.4354 & 0.2972 & 0.2936 & 0.4330 \\
\hline Subj10 & 1.0000 & 0.4416 & 0.4389 & 0.3565 & 0.3849 \\
\hline Subj11 & 1.0000 & 0.3376 & 0.3314 & 0.3810 & 0.4568 \\
\hline Subj12 & 1.0000 & 0.6158 & 0.5661 & 0.5132 & 0.4955 \\
\hline Subj13 & 1.0000 & 0.3097 & 0.3311 & 0.3711 & 0.3757 \\
\hline
\end{tabular}

For each negative match in table 5.13 the same subject signature is negatively matched in table 5.14, with the exception of subject 13 and his signature 2, this subject was correctly matched using the diffused template and signature. 
Table 5.14 Intra-subject similarity values. Diffused templates and signatures.

\begin{tabular}{|l|l|l|l|l|l|}
\hline & Template & Signature1 & Signature2 & Signature3 & Signature4 \\
\hline Subj01 & 1.0000 & 0.6006 & 0.5479 & 0.5273 & 0.5436 \\
\hline Subj02 & 1.0000 & 0.5091 & 0.4615 & 0.5159 & 0.5279 \\
\hline Subj03 & 1.0000 & 0.5997 & 0.3163 & 0.4769 & 0.5691 \\
\hline Subj04 & 1.0000 & 0.6466 & 0.5368 & 0.5922 & 0.3895 \\
\hline Subj05 & 1.0000 & 0.5044 & 0.5320 & 0.5787 & 0.5674 \\
\hline Subj06 & 1.0000 & 0.6407 & 0.7129 & 0.5733 & 0.6362 \\
\hline Subj07 & 1.0000 & 0.5144 & 0.5363 & 0.4874 & 0.4542 \\
\hline Subj08 & 1.0000 & 0.5807 & 0.5358 & 0.4684 & 0.4504 \\
\hline Subj09 & 1.0000 & 0.5295 & 0.3081 & 0.3026 & 0.5058 \\
\hline Subj10 & 1.0000 & 0.5319 & 0.5357 & 0.4373 & 0.4933 \\
\hline Subj11 & 1.0000 & 0.4332 & 0.4328 & 0.4449 & 0.5327 \\
\hline Subj12 & 1.0000 & 0.7569 & 0.7356 & 0.6799 & 0.6240 \\
\hline Subj13 & 1.0000 & 0.3527 & 0.4311 & 0.4331 & 0.4338 \\
\hline
\end{tabular}

In table 5.15 we present the percentage by which the similarity value increased by using diffused templates and signatures, negatively matched subjects are shown in red. An increase in the similarity value using diffused template and signatures does not translate in a positive match.

Table 5.15 Percentage increase on similarity values.

\begin{tabular}{|l|c|c|c|c|}
\hline & Signature & Signature2 & Signature3 & Signature4 \\
\hline T-Subj01 & $17.46 \%$ & $25.84 \%$ & $20.53 \%$ & $21.27 \%$ \\
\hline T-Subj02 & $19.17 \%$ & $21.04 \%$ & $24.44 \%$ & $22.09 \%$ \\
\hline T-Subj03 & $22.71 \%$ & $5.13 \%$ & $18.59 \%$ & $12.39 \%$ \\
\hline T-Subj04 & $23.98 \%$ & $22.47 \%$ & $22.22 \%$ & $18.39 \%$ \\
\hline T-Subj05 & $25.85 \%$ & $28.88 \%$ & $22.20 \%$ & $21.80 \%$ \\
\hline T-Subj06 & $21.29 \%$ & $18.56 \%$ & $20.53 \%$ & $17.45 \%$ \\
\hline T-Subj07 & $18.78 \%$ & $18.97 \%$ & $21.29 \%$ & $15.72 \%$ \\
\hline T-Subj08 & $19.96 \%$ & $18.79 \%$ & $19.49 \%$ & $17.96 \%$ \\
\hline T-Subj09 & $17.77 \%$ & $3.55 \%$ & $2.95 \%$ & $14.39 \%$ \\
\hline T-Subj10 & $16.97 \%$ & $18.06 \%$ & $18.48 \%$ & $21.96 \%$ \\
\hline T-Subj11 & $22.07 \%$ & $23.44 \%$ & $14.36 \%$ & $14.26 \%$ \\
\hline T-Subj12 & $18.63 \%$ & $23.04 \%$ & $24.51 \%$ & $20.58 \%$ \\
\hline T-Subj13 & $12.19 \%$ & $23.21 \%$ & $14.32 \%$ & $13.39 \%$ \\
\hline
\end{tabular}


Table 5.16 presents the overall results for the comparison of the signatures to the templates.

Four signatures for each subject were compared to the template and the average accuracy

of the match is reported for both the skeletonized and the diffused templates.

Table 5.16 Accuracy of matching for 4 distinct signatures taken at different time to the skeletonized and diffused templates in the database using Euclidean distances

\begin{tabular}{|l|l|l|l|l|l|l|}
\hline $\mathrm{D}_{i}$, Euclidean & $\mathrm{S}-1$ & $\mathrm{~S}-2$ & $\mathrm{~S}-3$ & $\mathrm{~S}-4$ & AVG. & $\sigma$ \\
\hline $\begin{array}{l}\text { Accuracy } \\
\text { (skeletonized) }\end{array}$ & $92.31 \%$ & $76.92 \%$ & $92.31 \%$ & $92.31 \%$ & $88.46 \%$ & 7.69 \\
\hline $\begin{array}{l}\text { Accuracy } \\
\text { (diffused) }\end{array}$ & $92.31 \%$ & $84.61 \%$ & $92.31 \%$ & $92.31 \%$ & $90.39 \%$ & 3.85 \\
\hline
\end{tabular}

Until now, the results presented were obtained using the Euclidean-based similarity measure in Eq. (4.1). We also calculated similarity values using the also well-known Manhattan metric, in Eq. (4.1) we simply substituted the Euclidean metric for the Manhattan metric, the procedure to calculate the similarity values was the same as described in chapter 4, similar tables as those presented in sections 5.2 and 5.3 were obtained. Table 5.17 presents the accuracy results obtained when comparing four signatures taken over time with the templates in our database using the Manhattan distance.

Table 5.17 Accuracy of matching for 4 distinct signatures taken at different time to the skeletonized and diffused templates in the database using Manhattan distances

\begin{tabular}{|l|c|c|c|c|c|c|}
\hline $\begin{array}{c}|c| \\
\mathrm{D}_{i}, \\
\text { Manhattan }\end{array}$ & S-1 & S-2 & S-3 & S-4 & AVG. & $\sigma$ \\
\hline $\begin{array}{l}\text { Accuracy } \\
\text { (skeletonized) }\end{array}$ & $100 \%$ & $76.92 \%$ & $92.31 \%$ & $92.31 \%$ & $90.39 \%$ & 9.68 \\
\hline $\begin{array}{l}\text { Accuracy } \\
\text { (diffused) }\end{array}$ & $100 \%$ & $76.92 \%$ & $92.31 \%$ & $92.31 \%$ & $90.39 \%$ & 9.68 \\
\hline
\end{tabular}

A paired two-tail student T-test was employed to determine if the accuracies obtained 
using the different distance methods are statistically different. It was found that the two distance measures do not yield statistically different results $(p=0.39)$.

In section 5.5 we present results obtained by performing experiments on the database C-X1obtained from the Computer Vision Research Laboratory at the University of Notre Dame [Flynn et al. 2003, Chen et al. 2003].

\subsection{Similarity Results for the C-X1 Database}

Once the merit of these similarity measures were confirmed within our database of subjects, other experiments were then conducted involving subjects in the C-X1 database.

The thermal images in this database, which consisted of 83 subjects each with four different poses, were collected using a Long-Wave Infrared (LWIR), non-cooled camera from Indigo systems. We could have used the entire data set, but for illustrative purposes, we first selected 6 different subjects and four thermal images for each. We applied the already described feature extraction algorithm to these new thermal images. We created a template from the facial signatures obtained for comparative purposes.

The challenge is viewed in this instance in the fact that the signatures obtained were in our opinion too noisy and may include features that are not necessarily part of the facial signature. Nonetheless our results are quite robust in including their database to prove the validity of the proposed approach, including the merit of the similarity measure. Using the Euclidean-based and Manhattan-based similarity measure we obtained results for comparing the templates and signatures from the $\mathrm{C}-\mathrm{X} 1$ data set to the templates in our 
database. When we compared template to template using the Euclidean- and Manhattan-based similarity measure the template matching was $100 \%$ accurate and none of the templates in our database were matched to the new templates from the $\mathrm{C}$-X1 database and vice versa. However when it came to match signatures to templates the new signatures were often mismatched with the templates in our database, the signatures in our database were never matched to the C-X1 templates. Based on these observations and results we chose randomly 25 subjects from the $\mathrm{C}-\mathrm{X} 1$ database and generated thermal signatures and templates for each subject, we proceeded to compute the similarity measure for skeletonized template and signature matching in the same fashion as with the subjects in our database. Table 5.18 presents the accuracy results obtained for skeletonized signatures and templates using the Euclidean- and Manhattan-based similarity measure.

Table 5.18 Accuracy matching for 4 distinct skeletonized signature sets and templates for 25 subjects in the $\mathrm{C}-\mathrm{X} 1$ database.

\begin{tabular}{|l|l|l|l|l|l|l|}
\hline & S-1 & S-2 & S-3 & S-4 & AVG. & $\sigma$ \\
\hline $\begin{array}{l}\text { Euclidean } \\
\text { (skeletonized) }\end{array}$ & $84 \%$ & $72 \%$ & $64 \%$ & $48 \%$ & $67 \%$ & 15.1 \\
\hline $\begin{array}{l}\text { Manhattan } \\
\text { (skeletonized) }\end{array}$ & $84 \%$ & $68 \%$ & $72 \%$ & $56 \%$ & $70 \%$ & 11.55 \\
\hline
\end{tabular}

The accuracy results for the subjects in the $\mathrm{C}-\mathrm{X} 1$ database are lower than the accuracy results obtained using our own database. The average accuracy for subjects in $\mathrm{C}-\mathrm{X} 1 \mathrm{is} 67 \%$ for the Euclidean-based similarity measure and 70\% for the Manhattan-based similarity measure, whereas the average accuracy for subjects in our database is $88.46 \%$ and $90.39 \%$ for the Euclidean- and Manhattan-based similarity measure respectively. The mismatch is 
inherent in the quality of the images. In communication with this research group we were informed that the images in $\mathrm{C}-\mathrm{X} 1$ contain raw measurements values from the sensor. Although the sensor is capable of being radio-metrically calibrated, the authors [Flynn et al. 2003, Chen et al. 2003] did not attempt to establish lookup tables that compute temperature from the sensor response, so the values provided in each LWIR thermograph is treated as an arbitrary temperature-correlated unit. The data-set in C-X1 also lacks non-uniformity correction (NUC), this is the correction of the non-uniform spread in gain and offset of the FPA detectors. Our method relies on the temperatures detected on the surface of the human skin so it is of great importance that calibration and NUC are performed in the infrared system. Typical thermal signatures of the C-X1 database are illustrated in figure 5.22 to demonstrate the complex nature of such signatures.
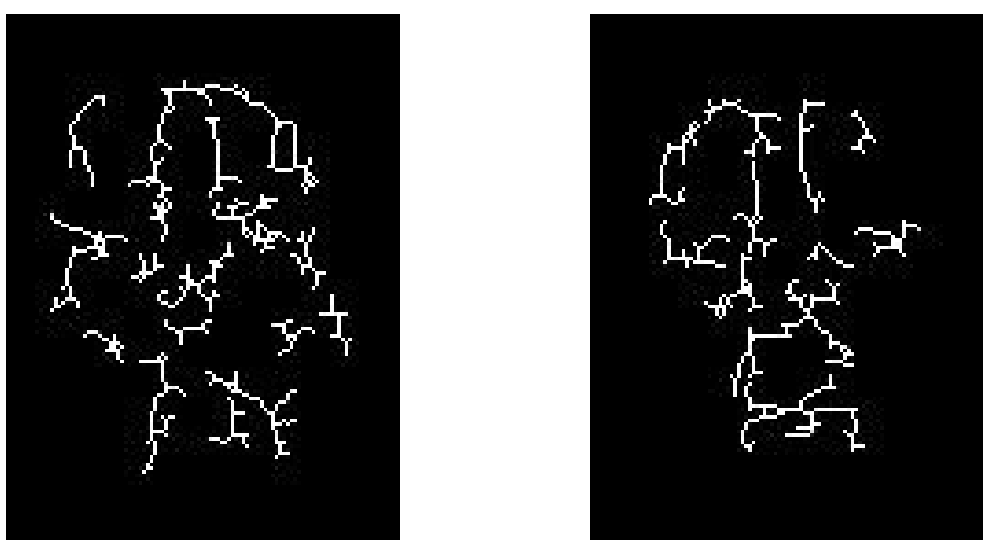

Fig. 5.22 Skeletonized signatures of two different subjects in C-X1.

In figure 5.23 we present two thermal signatures of subject 25 whose similarity results in a positive match to its template 

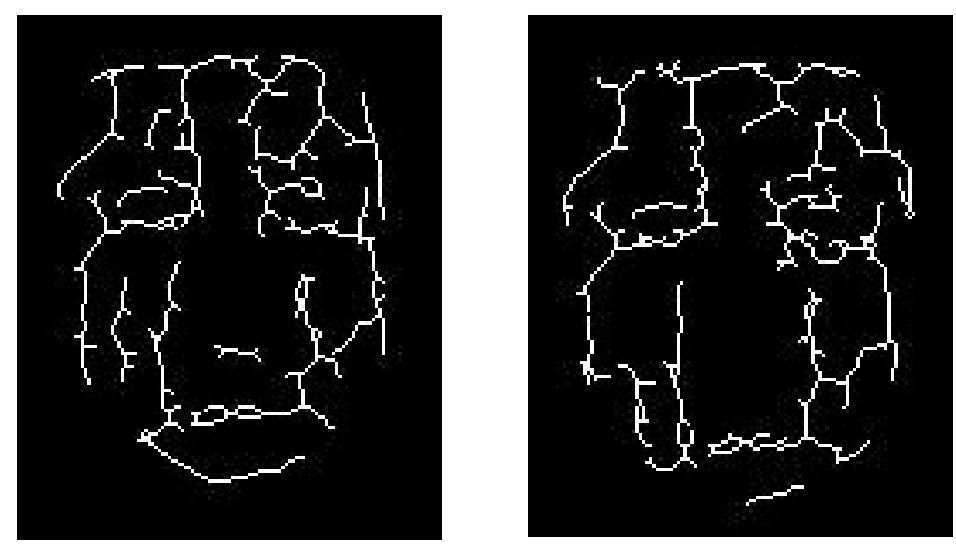

Fig. 5.23 Thermal signatures of a subject in dataset C-X1 and whose similarity values result in a positive match to its template

In figure 5.24 we present the overlay of the template and signature for the same subject as in figure 5.23, the template is shown in white and the signature is shown in red.
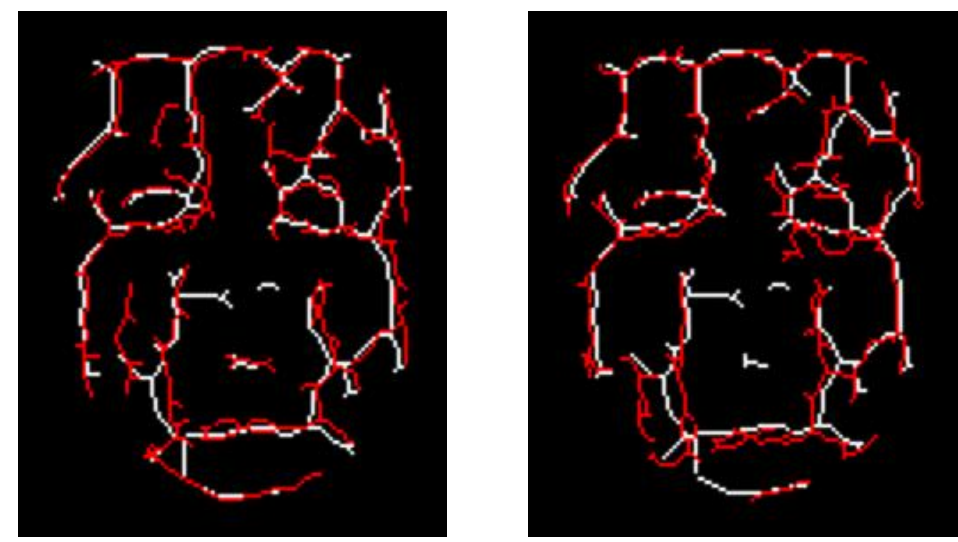

Fig. 5.24 Overlay of thermal signature (red) of a subject in dataset C-X1 and whose similarity values result in a positive match to its template (white)

Finally we present in figure 5.25 the overlay of the template and signature of subject 11 whose similarity value resulted in a negative match, in this case we present in figure $5.25 \mathrm{a}$ the overlay the template and signature subject 11 in this case the similarity value was not high enough to match the signature to its corresponding template, in figure $5.25 \mathrm{~b}$ we present the overlay of the signature of subject 11 and the template of subject 20 to which it was matched due to having the highest similarity between template and signature. 


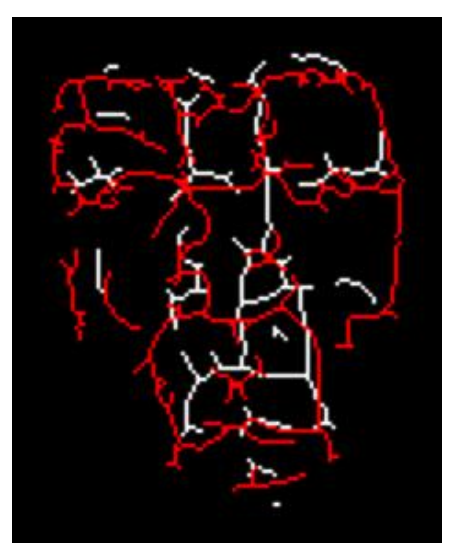

(a)

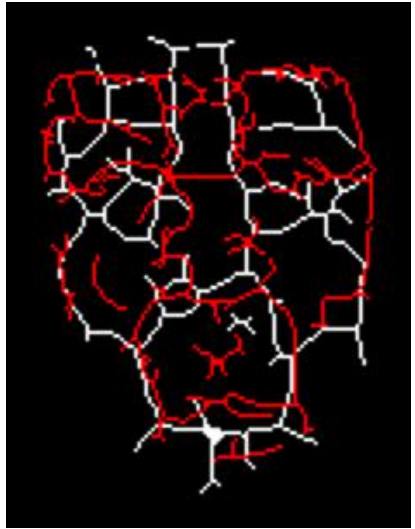

(b)

Fig. 5.25 Overlay of templates (white) and signatures (red) whose similarity values produced a negative match. The signature in $5.25 \mathrm{a}$ and $5.25 \mathrm{~b}$ is that of subject 11 .

\subsection{Validation of Similarity Values Using Principle Component Analysis}

\subsubsection{Introduction}

The principal component analysis (PCA) is a statistical technique widely used in the fields of pattern recognition, image compression, and decision making processes. In this study, it will be used as a validation technique to verify the results obtained by the similarity measure given in Eq. (4.1) but at the thermal images themselves rather than on the thermal signatures as was previously done with the C-X1 database.

Using our own database we have grouped the thermal IR images in 4 groups, each group is composed of 13 thermal IR images each one corresponding to a different subject. When validating the similarity results for group one, which corresponds to the signature set 1 , group 1 was considered the testing data set, and the other three groups are considered the training data set.

The main steps to perform the PCA are : 
1. Each thermal image was transformed into a $1 \mathrm{D}$ vector with $n$ pixels, where $n$ is determined by $M \times N$, and $M, N$ are the dimensions of the images (in our case, $M=256$, and $N=320$ ).

2. The $k$ vectors from the training images were organized into a 2D matrix $X$, where each vector constitutes a column $x_{i}$ in the matrix $\left(X=\left[x_{1}, x_{2}, x_{3}, \ldots, x_{k}\right]\right)$.

3. The mean vector $m$ across all training images were computed, where $=$ $1 / k \sum_{i=1}^{k} x_{i}$

4. The matrix $X$ was centered by subtracting the mean as $\bar{X}=\left[x_{1}-m, x_{2}-m, \ldots, x_{k}-m\right]$.

5. The covariance matrix $C_{X}$ of $X$ was calculated by $C_{X}=\bar{X} \bar{X}^{T}$.

6. Once the eigenvectors of the covariance matrix $C_{X}$ were calculated, the eigenvectors were sorted by the corresponding eigenvalues in a descending order to obtain the eigenvectors.

7. Select the first $q$ eigenvectors to form a feature basis $E=\left[e_{1}, e_{2}, e_{3}, \ldots, e_{q}\right]$.

8. Find the projections of each training image $p_{i}=E^{T}\left(x_{i}-m\right)$.

9. For the testing image, once it was transform to be a vector $y$, its projection is determined by $t=E^{T}\left(y_{i}-m\right)$.

10. Calculate the Euclidean distance by $d_{i}=\left|t-p_{i}\right|$.

11. Among the distance $\left[d_{1}, d_{2}, \ldots, d_{k}\right]$, find the minimum value $d_{s}$, and the $s^{\text {th }}$ image in the training set is determined as the recognition result for the testing image. 


\subsubsection{Results Obtained Using PCA}

The results obtained are shown in tables 5.19-5.22. The results for group 1 as the testing data set using PCA are shown in table 5.5.1, this group corresponds to the signature set 1.

Table 5.18 PCA results for group 1 as the testing data set, $\mathrm{q}=2$

\begin{tabular}{|l|l|l|l|l|l|l|l|l|l|l|l|l|}
\hline Subject ID & 1 & 2 & 3 & 4 & 5 & 7 & 8 & 9 & 10 & 11 & 12 & 13 \\
\hline $\begin{array}{l}\text { Recognized } \\
\text { subject ID }\end{array}$ & 1 & 2 & 3 & 4 & 5 & 7 & 8 & 9 & 10 & 11 & 12 & 13 \\
\hline
\end{tabular}

When using group 1 as the testing data set we found that the minimum distance between subject 13 and subject 12 is $0.12 \mathrm{E} 10+5$, which was not the minimum distance among all subjects. Using PCA we found that the overall minimum distance, $0.024 \mathrm{E} 10+5$, was obtained between thermal images of subject 13 . The similarity value between subject 13 and subject 12 has the highest value, 0.3108 and 0.3764 for skeletonized and diffused signature 1 (tables 5.2 and 5.7), thus incorrectly matching these two subjects.

The results for group 2 as the testing data set using PCA are shown in table 5.19, this group corresponds to thermal IR signatures 2 .

Table 5.19 PCA results for group 2 as the testing data set, $\mathrm{q}=6$

\begin{tabular}{|l|l|l|l|l|l|l|l|l|l|l|l|l|}
\hline Subject ID & 1 & 2 & 3 & 4 & 5 & 7 & 8 & 9 & 10 & 11 & 12 & 13 \\
\hline $\begin{array}{l}\text { Recognized } \\
\text { subject ID }\end{array}$ & 1 & 2 & 3 & 4 & 5 & 7 & 8 & 9 & 10 & 11 & 12 & 13 \\
\hline
\end{tabular}

Table 5.3 shows three cases in which subjects were incorrectly identified. The similarity value in table 5.3 between subject 3 and subject 12 has the highest value 0.3541 , using PCA subject 3 has a minimum distance of $3.14 \mathrm{E} 10+5$ to subject 12 , which was not the minimum distance among all subjects. The overall minimum PCA value $0.0078 \mathrm{E} 10+5$ was 
obtained between thermal images of subject 3 .

The similarity measure in table 5.3 between subject 9 and subject 2 has the highest value, 0.3233, using PCA subject 9 has a minimum distance $3.07 \mathrm{E} 10+5$ to subject 12 , which was not the minimum distance among all subjects. The overall minimum $0.0018 \mathrm{E} 10+5$ was obtained between thermal images of subject 9 .

The similarity value in table 5.3 between subject 13 and subject 7 has the highest value, 0.3356, using PCA subject 13 has a minimum distance $0.17 \mathrm{E} 10+6$ to subject 7 , which was not the minimum distance among all subjects. The overall minimum $0.018 \mathrm{E} 10+6$ was obtained between thermal images of subject 13 .

Table 5.8 shows two cases for which the thermal IR signature was incorrectly matched to its template, subject 3 and subject 9, these results cannot be confirmed through PCA validation. The PCA value for subject 3 , it is the same as the case described above.

The similarity value in table 5.8 between subject 9 and subject 6 has the highest value, 0.3776 , using PCA subject 9 has a minimum distance $1.66 \mathrm{E} 10+5$ to subject 6 , which was not the minimum distance among all subjects. The overall minimum $0.0018 \mathrm{E} 10+5$ was obtained between thermal images of subject 9 .

The results for group 3 as the testing data set using PCA are shown in table 5.20. Table 5.20 PCA results for group 3 as the testing data set, $\mathrm{q}=6$

\begin{tabular}{|l|l|l|l|l|l|l|l|l|l|l|l|l|}
\hline Subject ID & 1 & 2 & 3 & 4 & 5 & 7 & 8 & 9 & 10 & 11 & 12 & 13 \\
\hline $\begin{array}{l}\text { Recognized } \\
\text { subject ID }\end{array}$ & 1 & 2 & 3 & 4 & 5 & 7 & 8 & 9 & 10 & 11 & 12 & 13 \\
\hline
\end{tabular}

Using PCA subject 9 has a minimum distance value of $0.67 \mathrm{E} 10+6$ to subject 7 , which was 
not the minimum distance among all subjects. The overall minimum distance value of $0.0006 \mathrm{E} 10+6$ was obtained between thermal images of subject 9 . The similarity value between subject 9 and subject 7 is the highest in table 5.4 and 5.9, 0.3346 and 0.4072 respectively, these matching results cannot be replicated through PCA validation. The results for group 4 as the testing data set using PCA are shown in table 5.21. Table 5.21 PCA results for group 4 as the testing data set, $\mathrm{q}=8$

\begin{tabular}{|l|l|l|l|l|l|l|l|l|l|l|l|l|}
\hline Subject ID & 1 & 2 & 3 & 4 & 5 & 7 & 8 & 9 & 10 & 11 & 12 & 13 \\
\hline $\begin{array}{l}\text { Recognized } \\
\text { subject ID }\end{array}$ & 1 & 2 & 3 & 4 & 5 & 7 & 8 & 9 & 10 & 11 & 13 & 13 \\
\hline
\end{tabular}

Using similarity measure subject 4 is matched to subject 12 in table 5.5. Using PCA we find that subject 4 has a minimum distance $3.778 \mathrm{E} 10+5$ to subject 12 , which was not the minimum distance among all subjects. The overall minimum $0.0065 \mathrm{E} 10+5$ was obtained between thermal images of subject 4 . The similarity value in table 5.5 between subject 4 and subject 12 has the highest value, 0.3348 .

Using similarity measure subject 4 is matched to subject 6 in table 5.10. Using PCA we find that subject 4 has a minimum distance $1.405 \mathrm{E} 10+5$ to subject 6 , which was not the minimum distance among all subjects. The overall minimum $0.0065 \mathrm{E} 10+5$ was obtained between thermal images of subject 4 . The similarity value between subject 4 and subject 6 has the highest value, 0.4389 . When using group 4 as the testing data subject 12 was identified as subject 13 by PCA, while the similarity measure method correctly matched subject 12 in table 5.10. In cases in which PCA and the similarity measure method do not agree it is necessary to have other validation methods, such as manual inspection. 


\section{CHAPTER 6}

\section{Conclusion and Future Work}

The dissertation presented a novel approach for biometric facial recognition based on extracting features using thermal mid-wave infrared imaging. The approach used localized-contouring algorithms to segment the images of the face of the person and FSL for image registration. A morphological image processing technique was developed to extract the features from the thermal images, which were matched using a similarity measure based on the well known Euclidean and Manhattan metrics.

Thirteen subjects were used to create an in-house database and we successfully obtained the thermal infrared signatures and templates for the subjects using the proposed technique. The matching using the Euclidean-based similarity measure showed $88.46 \%$ accuracy for skeletonized feature signatures and $90.39 \%$ accuracy for diffused feature signatures. The matching using the Manhattan-based similarity measure showed $90.39 \%$ accuracy for both skeletonized and diffused feature signatures. Such high accuracies in the matching process clearly demonstrate the ability of the developed thermal infrared feature extraction and the distance-based similarity measure for accurate, low cost and effective subject matching. It has been demonstrated that by using diffused versions of the signatures and templates results yielded an $18.9 \%$ improvement in the similarity measures. This is a significant improvement in the similarities measures however a significant improvement on the detection accuracy was not achieved. Higher similarity measures are always essential in 
biometric matching processes and hence the diffused version of the matching process might be useful. However, if the system is aimed to be more restrictive and stringent the skeletonized version would be useful. The developed system creates highly distinct templates for the subject which contributes to the high accuracies in the results.

Other studies using thermal images have shown similar accuracies however they do not create time invariant templates as generated here. Our technique, which involved generation of a feature template by combining various images taken over time, ensures that minute changes in the vasculature over time may not impede in the matching process. The results section also demonstrated that the developed technique resulted in statistically indifferent results when different distance measures are used for the similarity technique. This shows that the signatures and the templates generated using the approach are robust enough so that any other similarity measures may also be employed.

The generalized structure of the proposed approach together with the uniqueness in the way thermal signature templates were generated and the similarity measure was formulated allows this approach to extend to other thermal images and databases. Caution should be taken however on what really constitutes a thermal pixel that is assumed to belong to the vasculature or at least be consistent through time using several thermal images. Thermal infrared image databases are available for research but the image quality in these databases is unsuitable for our purpose due to the lack of non-uniformity correction performed before gathering images which leads to erroneous feature extraction, 
other databases provide images of subjects in the outdoors and who are too far away from the camera to be able to extract a meaningful facial signature. Since these databases were not collected with the purpose of extracting features such as facial blood vessels patterns, future work would be to obtain a greater number of subjects to build a larger database for testing the algorithm.

The results also show that what is important in matching the thermal facial signature of a subject using a similarity measure is the ranking of the values. Also, PCA was used for validating the results of the similarity measure process. The validation provided good results with perfect matching in most of the cases. In cases where the validation provided results different from the ones obtained by the proposed approach, human intervention for the identification purpose is suggested.

Future work may also include the development of other image registration algorithms for improved and even more accurate registration than those acquired by FSL to increase the similarity values. Although finger print matching techniques have been used in the past for thermal feature matching, the deployment of such techniques on the present dataset must be accomplished to see if an improvement in the matching accuracy can be obtained. A graphical user interface (GUI) could also be developed in the future to assist in the thermal feature matching process. 


\section{LIST OF REFERENCES}

Adini, Y., Moses, Y., Ullman, S., "Face recognition: The Problem of Compensating for Changes In Illumination Direction", IEEE Transactions on Pattern Analysis and Machine Intelligence, Vol. 19, No. 7, pp. 721-732, July 1997.

Agarwal, K., Spyra, P.S., Beck, H., "Thermographic Imaging for Interventional Pain Management.” Proceedings Inframation, vol. 8, 2007, pp. 391-394.

Agarwal, K., Spyra, P.S., Beck, H., "Monitoring Effects of Sympathicolysis with Infrared-thermography in Patients with Complex Regional Pain Syndromes (CRPS)." Proceedings Inframation, vol. 9, 2008, pp. 7-10.

Aksoy, S., Haralick, R.M., "Feature Normalization and Likelihood-based Similarity Measures for Image Retrieval”, Pattern Recognition Letters, vol. 22, issue 5, 2001, pp. 563-582, ISSN 0167-8655, DOI: 10.1016/S0167-8655(00)00112-4.

Ashby,F.G., Ennis, D.M., Similarity measures. Scholarpedia, 2(12):4116, (2007), revision \#90369. doi:10.4249/scholarpedia.4116

Bebis, G., Gyaourova, A., Singh, S., and Pavlidis, I., "Face recognition by fusing thermal infrared and visible imagery", Image Vision Computing, vol. 24 , issue 7 (2006), pp. $727-$ 742.

Bhattacharyya, D., Rahul, R., Alisherov F. A., and Choi, M., "Biometric Authentication: A Review", International Journal of u- and e-Service, Science and Technology, vol. 2, No. 3, pp. 13-28, September, 2009.

Buddharaju, P., Pavlidis, I., Tsiamyrtzis, P., and Bazakos, M., "Physiology-based face recognition in the thermal infrared spectrum", IEEE Transactions on Pattern Analysis and Machine Intelligence, vol. 29, issue 4 (2007), pp. 613-626.

Candocia, F. M., Adjouadi, M., "A Similarity Measure For Stereo Feature Matching”, IEEE Transactions on Image Processing, vol.6, issue, 10, 1997, pp. 1460-1464.

Chekmenev, S., Farag, A., and Essock, E., "Thermal imaging of the superficial temporal artery: An arterial pulse recovery model", Proceedings of the 2007 IEEE Conference on Computer Vision and Pattern Recognition, Minneapolis, Minnesota, June 17-22 (2007).

Chen, X. , Flynn, P. J. , Bowyer, K. W. , "Visible -light and Infrared Face Recognition," 
ACM Workshop on Multimodal User Authentication, pp.48-55, December 2003.

Chen, Y; Adjouadi, M; Han, CA; Wang, J; Barreto, A; Rishe, N "A highly accurate and computationally efficient approach for unconstrained iris segmentation", Image and Vision Computing, 28 (2): 261-269, February 2010

Choi, S., S. Cha, Tappert, ,C. C., "A Survey of Binary Similarity and Distance Measures", Journal of Systemics, Cybernetics and Informatics, Vol. 8, No.1 2010, pp 43-48

Church, J.S., Cook, N. J., Schaefer, A.I., "Recent Applications of Infrared Thermography for Animal Welfare and Veterinary Research: Everything from Chicks to Elephants", Proceedings Inframation, vol. 10, 2009, pp. 215-224.

Delac, K., Grgic, M., "A survey of biometric recognition methods", Proceedings of 46 th International Symposium Electronics in Marine, 2004, pp. 184-193.

Flynn, P. J. , Bowyer, K. W. , Phillips, P. J., "Assessment of time dependency in face recognition: An initial study," Audio- and Video-Based Biometric Person Authentication, pp.44-51, 2003.

Friedrich, G., and Yeshurun, Y., "Seeing People in the Dark: Face Recognition in Infrared Images", Biologically Motivated Computer Vision, 2002: 348-359.

Guzman, A., Adjouadi,M., Goryawala,M., "Detecting the Human Face Thermal signature Using Thermal Infrared Imaging", CAHSI 4th. Annual Meeting, Redmond, WA.; April 5, 2010 .

Hernández, B., Olague, G., Hammoud, R., Trujillo, L., and Romero, E., "Visual learning of texture descriptors for facial expression recognition in thermal imagery", Computer Vision and Image Understanding, 106 2-3 (2007), pp. 258-269.

Hidalgo, Juan C., "Detecting H1N1 in Tocumen International Airport in Panama", Application Note for Research and Scienc, FLIR Technical Series.www.flir.com/WorkArea/DownloadAsset.aspx?id=50052

Hua Li; Yezzi, A.; , "Local or Global Minima: Flexible Dual-Front Active Contours," Pattern Analysis and Machine Intelligence, IEEE Transactions on, vol.29, no.1, pp.1-14, Jan. 2007 doi: 10.1109/TPAMI.2007.250595

Im, S.K. , Choi, H.S. , Kim, S.W. , "A Direction-based Vascular Pattern Extraction 
Algorithm for Hand Vascular Pattern Verification”, ETRI Journal, Volume 25, Number 2, pp. 101-108, April 2003.

Jain, A.K., Flynn,P., Ross,A.A., Handbook of Biometrics. New York: Springer, 2008.

Jain, R.; Murthy, S.N.J.; Chen, P.L.-J.; Chatterjee, S.; , "Similarity measures for image databases," ., Proceedings of 1995 Fourth IEEE International Conference on Fuzzy Systems and The Second International Fuzzy Engineering Symposium, vol.3, no., pp.1247-1254 vol.3, 20-24 Mar 1995

Jones, B. F., and Plassmann, P., "Digital Infrared Thermal Imaging of Human Skin Computational Approaches to Image Processing for Improved Interpretation and Analysis", IEEE ENGINEERING IN MEDICINE AND BIOLOGY, November/December 2002

Kruse, P.W., MxGlauchlin, L.D., MxQuistan, R.B., “Elements of Infrared Technology”, Wiley, New York, 1962.

Lankton, S. , Tannenbaum, A. , "Localizing Region-Based Active Contours", IEEE Transactions on Image Processing, Vol. 17, No. 11, pp. 2029-2039, November 2008.

Lesot,M-J., Rifqi, M.H., Benhadda,H.," Similarity measures for binary and numerical data: a survey",International Journal of Knowledge Engineering and Soft Data Paradigms, Vol. 1, No.1, pp. 63 - 84, 2009.

Lin, C-L., Fan, K-C., "Biometric verification using thermal images of palm-dorsa vein patterns," IEEE Transactions onCircuits and Systems for Video Technology, vol.14, no.2, pp. 199- 213, Feb. 2004. doi: 10.1109/TCSVT.2003.821975

Manohar, C., Extraction of Superficial Vasculature in Thermal Imaging, Master's Thesis, University of Houstong, TX, 2004.

MeihuaBao; SiyuGuo; Qiu Tang; Fan Zhang; , "Optimization of the bwmorph Function in the MATLAB Image Processing Toolbox for Binary Skeleton Computation," Computational Intelligence and Natural Computing, 2009. CINC '09. International Conference on, vol.2, no., pp.273-276, 6-7 June 2009

doi: $10.1109 /$ CINC.2009.96

Maltoni, D., Maio, D., Jain, A. K., Prabhakar, S., 2009. Handbook of Fingerprint Recognition (2nd ed.). Springer Publishing Company, Incorporated. 
Mital, M. and Scott, E., "Thermal Detection of Embedded Tumors Using Infrared Imaging," Journal of Biomechanical Engineering, Vol. 129, February 2007, 33-39.

Miura, N., Nagasaka, A., Miyatake, T., "Feature Extraction of Finger Vein Patterns Based On Iterative Line Tracking and its Application to Personal Identification", Machine Vision and Applications, Volume 15, No. 4, pp. 194-203, October, 2004.

Moxham, B.J., Kirsh, C., Berkovitz, B., Alusi, G., and Cheeseman, T., Interactive Head and Neck (CD-ROM). Primal Pictures, Dec. 2002.

Pavlidis, I., Dowdall, J., Sun, N., Puri, C., Fei, J., and Garbey, M., "Interacting with human physiology", Computer Vision and Image Understanding 108(1-2), 150-170 (2007).

Pavlidis, I., Eberhardt, N., and Levine, J. A., "Seeing through the face of deception", Nature, 2002 Feb 7.

Pavlidis, I., and Levine, J., "Thermal Image Analysis for Polygraph Testing", IEEE Engineering in Medicine and Biology, November/December 2002.

Pavlidis, I. , Tsiamyrtzis, P. , Buddhraju, P. , Manohar, C. , "Biometrics: Face Recognition

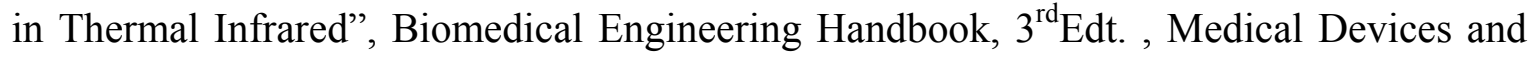
Systems, Chp. 29, 2006.

Perona,P. and Malik, J., "Scale-space and edge detection using anisotropic diffusion", IEEE Trans. Pattern Analysis and Machine Intelligence, vol. 12-7 (1990), pp. 629-639.

Puri, A., Olson, L., Pavlidis, I., Levine, J., and Starren, J., "Stresscam: non-contact measurement of users' emotional states through thermal imaging", Proceedings of the 2005 ACM Conference on Human Factors in Computing Systems (CHI), Portland, OR, 2005, pp. 1725-1728.

Qi, H., and Diakides N. A., "Infrared imaging in Medicine", CRC Press, Boca Raton, Florida, USA, 2007.

Ratha, N. K., Govindaraju,V., Advances in Biometrics: Sensors, Algorithms and Systems. London: Springer, 2008. 
Reese, J., "Applications Of Infrared Imagery In The Monitoring And Diagnosis Of Zoo Animals In Anchorage, Alaska”, Proceedings Inframation, vol.7, 2006, pp. 145-150.

Ring, F.E., Ammer,K., "Thermal Imaging in Diseases of the Skeletal and Neuromuscular Systems", Medical Infrared Imaging, Editors: Nicholas A. Diakides, Joseph D. Bronzino, CRC Press, Boca Raton, FL; 2008.

Rogalski, A., "Infrared detectors: an overview", Infrared Physics \& Technology, 43 (2002) 187-210.

Rogalski, A. , "Recent progress in infrared detector technologies", Infrared Physics \& Technology, Volume 54, Issue 3, May 2011, Pages 136-154, ISSN 1350-4495, 10.1016/j.infrared.2010.12.003.

Rogalski, A., and Chrzanowski, K., "Infrared devices and techniques",Opto-electronics Review, 10(2), 111-136 (2002).

Santini, S.; Jain, R.; , "Similarity measures," Pattern Analysis and Machine Intelligence, IEEE Transactions on, vol.21, no.9, pp.871-883, Sep 1999

doi: $10.1109 / 34.790428$

Shimooka, T. , Shimizu, K., "Artificial Immune System For Personal Identification With Finger Vein Pattern”, M.Gh. Negoita et al.. (Eds.): KES 2004, LNAI 3214, pp. 511-518, 2004.

Smith, S.M., M. Jenkinson, M.W. Woolrich, C.F. Beckmann, T.E.J. Behrens, H. Johansen-Berg, P.R. Bannister, M. De Luca, I. Drobnjak, D.E. Flitney, R. Niazy, J. Saunders, J. Vickers, Y. Zhang, N. De Stefano, J.M. Brady, and P.M. Matthews. "Advances in functional and structural MR image analysis and implementation as FSL", NeuroImage, 23(S1):208-219, 2004.

Smith, R.A., Jones, F.E., Chasmar, R.P., "The Detection and measurement of Infrared Radiation”, Clarendon, Oxford, 1958.

Socolinsky, D.A., Selinger, A. and Neuheisel, J.D., "Face recognition with visible and thermal infrared imagery", Computer Vision Image Understanding, Volume 91, Issues 1-2,(2003), pp. 72-114.

Shimooka, T. , Shimizu, K., "Artificial Immune System For Personal Identification With Finger Vein Pattern", M.Gh. Negoita et al.. (Eds.): KES 2004, LNAI 3214, pp. 511-518, 
2004.

Straume, A., Oftedal,, G., Johnson, A., "Skin Temperature Increase Caused by a Mobile Phone: A Methodological Infrared Camera Study," Bioelectromagnetics26(2005), 510-510.

Vollmer, M., Möllmann, K-P.,Infrared Thermal ImagingFundamentals, Research and Applications,Wiley-VCH; 1 edition (October 12, 2010).

Veltkamp,R.C., "Shape Matching: Similarity Measures and Algorithms", International Conference on Shape Modeling and Applications, p.0188, 2001

Wang, S., Lv, S., Wang, X., "Infrared Facial Expression Recognition Using Wavelet Transform", International Symposium on Computer Science and Computational Technology, vol. 2, pp.327-330, 2008. DOI: 10.1109/ISCSCT.2008.356.

Wayman, J., Jain, A., Maltoni, D., Maio, D., Biometric Systems: Technology, Design, and Performance Evaluation. London: Springer, 2005.

Woolrich, M.W., Jbabdi, S., Patenaude, B., Chappell, M., Makni, S., Behrens, T., Beckmann, C., Jenkinson, M., Smith, S.M., "Bayesian analysis of neuroimaging data in FSL. NeuroImage", 45:S173-186, 2009.

Zeng,Z., "Spontaneous Emotional Facial Expression," Detection,Journal of Multimedia, Vol.1(5): 1-8, 2006.

Zhao, W., Chellappa, R. and Rosenfeld, A., "Face recognition: a literature survey", $A C M$ Computing Surveys, 35 December (2003), pp. 399-458.

Zhu. Z., Tsiamyrtzis, P., and Pavlidis I., "The Segmentation of the Supraorbital Vessels in Thermal Imagery", Proceedings of the 2008 IEEE Fifth International Conference on Advanced Video and Signal Based Surveillance, pp.237-244, September 01-03, 2008.

User's Guide Merlin ${ }^{\mathrm{TM}}$ Mid InSb MWIR Camera, Version 120, 414-0001-10.

ThermaCAM ${ }^{\mathrm{TM}}$ Researcher. User's manual. Publ. No. 1558 071. November 9, 2004.

The Ultimate Resource Infrared Handbook for R\&D Professionals. FLIR Systems. 


\section{VITA}

$\begin{array}{ll} & \text { ANA M. GUZMÁN TAMAYO } \\ 2002 & \text { B.S., Computer Engineering } \\ & \text { Southern Methodist University } \\ & \text { Dallas, Texas., USA } \\ 2006 & \text { M.S., Computer Engineering } \\ & \text { Florida International University } \\ & \text { Miami, Florida., USA } \\ & \text { Ph.D., Electrical Engineering } \\ & \text { Florida International University } \\ & \text { Miami, Florida }\end{array}$

HONORS AND AWARDS

Florida International University, Research Assistant (2005 - 2011)

\section{EXPERIENCE}

International Business Machines (IBM)

Thomas J. Watson Research Center - Yorktown Heights,NY

Summer Intern - Picosecond Imaging Circuit Analysis group May-August 2007

- Successfully built an inline spectrometer for photon counting detectors, to understand the relationship between technology parameters such as channel length and Voltage and the wavelength of the emission light as well as to ascertain the spectrum of leakage emission and switching emission light in circuits.

- Performed Infrared alignment of the spectrometer

- Automated the spectrometer using MATLAB and GPIB

COMMUNITY INVOLVEMENT

- Volunteer Spanish translator for Friends of the Orphans 2005-present

- Volunteer teacher of ESL, Public Library in Key Biscayne, FL 2003-2006

- Volunteer at the Pacific Life Open Professional Tennis Tournament 2002

- Volunteer at Infinity Project, Southern Methodist University 2001-2002

- Volunteer at Charities Home Center in Dallas, TX 2001-2002

EXTRA-CURRICULAR ACTIVITIES

- Delta Epsilon Iota - Academic Honor Society

2006-present

- Society of Hispanic Professional Engineers (SHPE) at FIU 2003-present

- Director, CpE Group,Women in Science and Engineering at FIU 2003- 2004 
- Chair, Association of Computer Machinery (ACM) at SMU 2001- 2002

- Community Director Women in Science and Engineering at SMU 2000-2002

- Society of Women Engineers (SWE) at SMU

1999-2002

\section{PUBLICATIONS AND PRESENTATIONS}

1. A. Guzman, M. Goryawala, and M. Adjouadi, "Generating Facial Vasculature Signatures Using Thermal Infrared Images", Proceedings of the $5^{\text {th }}$ Annual CAHSI Workshop, San Juan, Puerto Rico, pp. 31-34, March 27-29, 2011.

2. A. Guzman, M. Goryawala, and M. Adjouadi, "Detecting the Human Face Vasculature Using Thermal Infrared Imaging", Proceedings of the $4^{\text {th }}$ Annual CAHSI Workshop, Microsoft Headquarters, Redmond, Washington, pp. 73-76, April 5-7, 2010.

3. A. Guzman, M. Adjouadi, L. Wang, A. Barreto, N. Rishe, "Thermal Infrared Imaging for the Detection of Temperature Increase on the Head Surface Due to Motor Activity", Poster Presentation, p.91, SACNAS National Conference Abstracts 2009, Dallas, Texas, October 15-18, 2009.

4. A. Guzman, M. Adjouadi, L. Wang, A. Barreto, N. Rishe, "Thermal Infrared Imaging for the Detection of Temperature Increase on the Head Surface Due to Motor Activity", Proceedings of the CAHSI Annual Meeting, pp. 30-33, Jan 15-18, 2009, Google headquarters, Mountain View, CA.

5. A. Guzman and M. Adjouadi, "Inline Spectrometer For Photon Counting Detectors", pp. 41-43, Proceedings of the $2^{\text {nd }}$ Annual CAHSI Workshop, Miami, Florida, December 15-18, 2007.

6. A. Guzman, M. Goryawala, M. Adjouadi, "Generating Thermal Facial Signatures Using Thermal Infrared Images', accepted, 2012 IEEE International Conference on Emerging Signal Processing Applications, Las Vegas, Nevada.

7. A. Guzman, M. Goryawaya, J.Wang, A. Barreto, N. Rishe, J. Andrian, M.Adjouadi, "Feature Matching and Similarity Measurements in Thermal Imaging for Facial Signature Authentication", submitted, IEEE Transactions on Information Technology in BioMedicine, Oct 2011.

8. A. Guzman, J. Wang, M. Goryawala, A. Barreto, N. Rishe, J. Andrian, M. Adjouadi "An Integrated Approach to Face Recognition Using Thermal Imaging", submitted to the Int'l Conference on Information Visualization Theory and Applications (IVAPP 2012), Rome, Italy. 